\title{
CURRENT TRENDS AND OPEN PROBLEMS IN ARITHMETIC DYNAMICS
}

\author{
ROBERT BENEDETTO, PATRICK INGRAM, RAFE JONES, MICHELLE MANES, \\ JOSEPH H. SILVERMAN, AND THOMAS J. TUCKER
}

Abstract. Arithmetic dynamics is the study of number theoretic properties of dynamical systems. A relatively new field, it draws inspiration partly from dynamical analogues of theorems and conjectures in classical arithmetic geometry and partly from $p$-adic analogues of theorems and conjectures in classical complex dynamics. In this article we survey some of the motivating problems and some of the recent progress in the field of arithmetic dynamics.

\section{Contents}

1. Introduction

2. Abstract dynamical systems

3. Background: Number theory and algebraic geometry

4. Uniform boundedness of (pre)periodic points

5. Arboreal representations

6. Dynatomic representations

7. Intersections of orbits and subvarieties

8. (Pre)periodic points on subvarieties

9. Dynamical (dynatomic) modular curves

10. Dynamical moduli spaces

11. Unlikely intersections in dynamics

12. Good reduction of maps and orbits

13. Dynamical degrees of rational maps

14. Arithmetic degrees of orbits

15. Canonical heights

16. Variation of the canonical height

17. $p$-adic and non-archimedean dynamics

18. Dynamics over finite fields

19. Irreducibilty and stability of iterates

20. Primes, prime divisors, and primitive divisors in orbits

21. Integral points in orbits

Received by the editors June 30, 2018.

2010 Mathematics Subject Classification. Primary 37P05; Secondary 37P15, 37P20, 37P25, 37P30, 37P45, 37P55.

Key words and phrases. Arithmetic dynamics, open problems.

The first author's research was supported by NSF Grant DMS-1501766.

The fourth author's research was supported by Simons Collaboration Grant \#359721.

The fifth author's research was supported by Simons Collaboration Grant \#241309.

The sixth author's research was supported by NSF Grant DMS-0101636. 
22. Local-global questions in dynamics

Acknowledgments

About the authors

\section{INTRODUCTION}

In classical real and complex dynamics, one studies topological and analytic properties of orbits of points under iteration of self-maps of $\mathbb{R}$ or $\mathbb{C}$, or more generally self-maps of real or complex manifolds. In arithmetic dynamics, a more recent subject, one likewise studies properties of orbits of self-maps, but with a number theoretic flavor. In this article we describe some of the motivating problems and recent advances in this relatively new field of arithmetic dynamics.

Many of the motivating problems in arithmetic dynamics come via analogy from classical problems in arithmetic geometry. A large part of arithmetic geometry involves studying rational and integral points on algebraic varieties, and it is fair to say that many deep results in the subject involve abelian varieties, which are algebraic varieties admitting a group structure. Table1 provides a "dictionary" that suggests ways in which classical theorems and conjectures in arithmetic geometry lead naturally to dynamical problems of an arithmetic nature.

TABLE 1. An arithmetical/dynamical dictionary [218, §6.5]

Arithmetic Geometry Dynamical Systems

\begin{tabular}{|c|c|}
\hline $\begin{array}{l}\text { rational and integral } \\
\text { points on varieties }\end{array}$ & $\begin{array}{l}\text { rational and integral } \\
\text { points in orbits }\end{array}$ \\
\hline $\begin{array}{l}\text { torsion points on } \\
\text { abelian varieties }\end{array}$ & $\begin{array}{l}\text { periodic and preperiodic } \\
\text { points of rational maps }\end{array}$ \\
\hline $\begin{array}{l}\text { abelian varieties with } \\
\text { complex multiplication }\end{array}$ & $\begin{array}{l}\text { post-critically finite } \\
\text { rational maps }\end{array}$ \\
\hline
\end{tabular}

A second source of inspiration for arithmetic dynamics comes from a basic principle in number theory: before studying a problem over the field of rational numbers $\mathbb{Q}$, one should first study it over all completions of $\mathbb{Q}$, including in particular $p$-adic fields. The field $\mathbb{Q}_{p}$ of $p$-adic rationals comes equipped with an absolute value, so many concepts from complex dynamics can be translated to the $p$-adic setting, and one can try to prove $p$-adic analogues of complex dynamical theorems. However, since the $p$-adic absolute value satisfies the non-archimedean triangle inequality $\|x+y\| \leq \max \{\|x\|,\|y\|\}$, there are striking differences between the $p$-adic and complex theories, many of them traceable to two facts:

(1) $p$-adic fields are totally disconnected;

(2) the smallest complete algebraically closed field containing $\mathbb{Q}_{p}$ is not locally compact.

Despite, or possibly because of, these differences, the study of $p$-adic dynamics is a thriving branch of arithmetic dynamics. 
The goal of this article is to describe motivating problems and conjectures in the field of arithmetic dynamics and to give a brief description of recent progress. We start in Sections 2 and 3 with an overview of the terminology, notation, and background on dynamical systems, number theory, and algebraic geometry that we will need. As noted at the end of Section 3. we have attempted to make this article accessible by generally restricting our attention to maps of projective space, thereby obviating the need for more advanced material from algebraic geometry. Our survey of arithmetic dynamics then commences in Section 4 and concludes in Section 22. These 19 sections are mostly independent of one another, albeit liberally sprinkled with instructive cross-references.

A brief timeline of the early days. The study of Galois groups of iterated polynomials was initiated by Odoni in a series of papers [182 184] during the mid-1980s. Beyond this, it appears that the first paper to describe dynamical analogues for a wide range of classical problems in arithmetic geometry did so not for polynomial maps or projective space, but rather for K3 surfaces admitting an automorphism of infinite order. The 1991 paper of Silverman [209] discusses dynamical analogues of various problems, including

(1) uniform boundedness of periodic points,

(2) periodic points on subvarieties,

(3) lower bounds for canonical heights,

(4) open image of Galois groups,

(5) integral points in orbits.

The rest of the 1990s saw an explosion of papers in which numerous researchers began to study a wide variety of problems in arithmetic dynamics.

The serious study of $p$-adic dynamics starts with a 1983 paper of Herman and Yoccoz [109], two of the world's leading complex dynamicists, in which they investigate a $p$-adic analogue of a classical problem in complex dynamics. Later in the 1980s and 1990s, the physics literature includes several papers [6, 7, 21, 236] that discuss $p$-adic dynamics for polynomial maps, but the deeper study of $p$-adic dynamics really took off with the PhD theses of Benedetto [24, 25] in 1998 and Rivera-Letelier [195, 196] in 2000. The dynamics of iterated $p$-adic power series was investigated by Lubin [160 in a 1994 paper. We also mention that there is an extensive literature on ergodic theory over $p$-adic fields which, although not within the purview of this survey, dates back to at least 1975 [185].

\section{Abstract Dynamical Systems}

A discrete dynamical system consists of a set $S$ and a self-map

$$
f: S \longrightarrow S \text {. }
$$

We write

$$
f^{n}:=\underbrace{f \circ f \circ \cdots \circ f}_{n \text { copies }}
$$

for the $n$th iterate of $f$ with the convention that $f^{0}$ is the identity map, and we write

$$
\mathcal{O}_{f}(\alpha):=\left\{f^{n}(\alpha): n \geq 0\right\}
$$

for the forward orbit of an element $\alpha \in S$. A fundamental problem of discrete dynamics is to classify the points in $S$ according to the behavior of their orbits. 
Frequently, this behavior is described according to some additional properties of the set $S$, which for example might be a topological space or a metric space or an analytic space. But even in the setting of abstract sets, we can make interesting distinctions among orbits.

Definition 2.1. Let $f: S \rightarrow S$ be a dynamical system, and let $\alpha \in S$. We say that $\alpha$ is

$$
\begin{aligned}
\text { wandering }_{\frac{1}{1}} & \text { if } \mathcal{O}_{f}(\alpha) \text { is infinite, } \\
\text { preperiodic } & \text { if } \mathcal{O}_{f}(\alpha) \text { is finite, } \\
\text { periodic } & \text { if } f^{n}(\alpha)=\alpha \text { for some } n \geq 1 .
\end{aligned}
$$

If $\alpha$ is periodic, the smallest $n \geq 1$ satisfying $f^{n}(\alpha)=\alpha$ is called the (exact) period of $\alpha$. We set the useful notation

$$
\begin{aligned}
\operatorname{PrePer}(f, S) & :=\left\{\alpha \in S: f^{n}(\alpha)=f^{m}(\alpha) \text { for some } n>m \geq 0\right\}, \\
\operatorname{Per}(f, S) & :=\left\{\alpha \in S: f^{n}(\alpha)=\alpha \text { for some } n \geq 1\right\}, \\
\operatorname{Per}_{n}(f, S) & :=\left\{\alpha \in S: f^{n}(\alpha)=\alpha\right\}, \\
\operatorname{Per}_{n}^{*}(f, S) & :=\left\{\alpha \in S: f^{n}(\alpha)=\alpha \text { and } f^{i}(\alpha) \neq \alpha \text { for all } 1 \leq i<n\right\} .
\end{aligned}
$$

Thus, $\operatorname{PrePer}(f, S)$ is the set of preperiodic points, $\operatorname{Per}(f, S)$ is the set of periodic points, $\operatorname{Per}_{n}(f, S)$ is the set of periodic points of period dividing $n$, and $\operatorname{Per}_{n}^{*}(f, S)$ is the set of periodic points of period exactly equal to $n$.

In this way the map $f$ partitions the points of $S$ into two subsets depending on whether a point's orbit is finite or infinite, and it further partitions the former into two subsets depending on whether a point's orbit is strictly periodic. More generally, if $S$ has additional structure, for example if $S$ is a complex manifold and $f$ is analytic, one classically partitions $S$ into the Fatou set, consisting of points where the iterates of $f$ behave nicely, and the complementary Julia set, consisting of points where the iterates of $f$ behave chaotically.

Example 2.2. Let $G$ be a group, let $d \geq 2$, and let $f: G \rightarrow G$ be the $d$ th power map $f(\gamma)=\gamma^{d}$. Then it is a fun exercise to verify that

$$
\operatorname{PrePer}(f, G)=\{\gamma \in G: \gamma \text { has finite order }\} \text {. }
$$

Moving to the next level, dynamicists often study collections of maps. Suppose that $\mathcal{F}$ is a collection of maps $f: S \rightarrow S$. Then a basic metaproblem is to classify the maps $f$ in $\mathcal{F}$ according to the dynamical behavior of $f$. Here is a typical example.

Question 2.3. Let $S$ be a set, and let $T \subseteq S$ be a subset. Classify those maps $f: S \rightarrow S$ with the property that for every $\alpha \in S$, the set

$$
\left\{n \geq 0: f^{n}(\alpha) \in T\right\}
$$

has some nice structure, for example, it is the union of finitely many one-sided arithmetic progressions.

\footnotetext{
${ }^{1}$ We mention that our definition of "wandering point" is different from the classical definition for dynamical systems on topological, respectively measure, spaces, where $\alpha$ is said to be wandering if there is a neighborhood $U$ of $\alpha$ such that $f^{n}(U) \cap U$ is empty, respectively has measure 0 , for all sufficiently large $n$.
} 


\section{BaCkground: Number theOry AND Algebraic GeOMEtry}

In this paper we use $\mathbb{Z}, \mathbb{Q}, \mathbb{R}, \mathbb{C}$ as usual to denote the integers, rational numbers, real numbers, and complex numbers. We write $\mathbb{F}_{q}$ for a finite field with $q$ elements. We fix an algebraic closure $\overline{\mathbb{Q}}$ for $\mathbb{Q}$. Elements of $\overline{\mathbb{Q}}$ are called algebraic numbers. A number field is a finite extension $K$ of $\mathbb{Q}$.

The completions of $\mathbb{Z}$ and $\mathbb{Q}$ for their $p$-adic valuations are denoted $\mathbb{Z}_{p}$ and $\mathbb{Q}_{p}$, respectively, and a completion of an algebraic closure of $\mathbb{Q}_{p}$ is denoted $\mathbb{C}_{p}$.

At times we will want a way to measure the size of an infinite collection of primes.

Definition 3.1. The (natural) density of a set of primes $\mathcal{P} \subset \mathbb{Z}$ is the following quantity, provided that the limit exists:

$$
\operatorname{Density}(\mathcal{P})=\lim _{X \rightarrow \infty} \frac{\#\{p \in \mathcal{P}: p \leq X\}}{\#\{\text { primes } p: p \leq X\}} .
$$

Let $N \geq 1$. For a given field $K$, we write $\bar{K}$ to denote a separable closure of $K 2$ Projective $N$-space (over $K$ ), denoted $\mathbb{P}^{N}$, is the quotient space

$$
\mathbb{P}^{N}:=\left(\bar{K}^{N+1} \backslash \mathbf{0}\right) / \bar{K}^{*},
$$

where the group $\bar{K}^{*}$ acts on $(N+1)$-tuples via scalar multiplication. If we want to specify that the coordinates of the $(N+1)$-tuples are in the field $K$, we write

$$
\mathbb{P}^{N}(K):=\left(K^{N+1} \backslash \mathbf{0}\right) / K^{*} .
$$

We write

$$
\left[a_{0}, a_{1}, \ldots, a_{N}\right] \in \mathbb{P}^{N}
$$

to denote the point in $\mathbb{P}^{N}$ corresponding to the $(N+1)$-tuple $\left(a_{0}, \ldots, a_{N}\right)$. The field of definition $K(P)$ of a point $P=\left[a_{0}, \ldots, a_{N}\right] \in \mathbb{P}^{N}$ is defined by choosing any index $i$ such that $a_{i} \neq 0$ and setting

$$
K(P):=K\left(a_{0} / a_{i}, a_{1} / a_{i}, \ldots, a_{N} / a_{i}\right) .
$$

The Galois group $\operatorname{Gal}(\bar{K} / K)$ acts on $\mathbb{P}^{N}$ via its action on the coordinates of an $N$-tuple, and it is a consequence of Hilbert's Theorem 90 that

$$
\mathbb{P}^{N}(K)=\left\{P \in \mathbb{P}^{N}: \sigma(P)=P \text { for all } \sigma \in \operatorname{Gal}(\bar{K} / K)\right\} .
$$

More generally, the field of definition of $P$ may be defined Galois-theoretically as

$$
K(P):=\text { subfield of } \bar{K} \text { fixed by }\{\sigma \in \operatorname{Gal}(\bar{K} / K): \sigma(P)=P\} .
$$

We next discuss maps from $\mathbb{P}^{N}$ to itself. A rational map $f: \mathbb{P}^{N} \rightarrow \mathbb{P}^{N}$ is specified by an $(N+1)$-tuple of homogeneous polynomials $f_{0}, \ldots, f_{N} \in \bar{K}\left[X_{0}, \ldots, X_{N}\right]$ of the same degree,

$$
f=\left[f_{0}, \ldots, f_{N}\right]: \mathbb{P}^{N}--\rightarrow \mathbb{P}^{N} .
$$

Since the polynomial ring $\bar{K}\left[X_{0}, \ldots, K_{N}\right]$ is a unique factorization domain, we always cancel any factors common to all of $f_{0}, \ldots, f_{N}$. With this proviso, the degree of $f$ is defined to be the common degree of the polynomials $f_{0}, \ldots, f_{N}$. The map $f$ is said to be dominant if the polynomials $f_{0}, \ldots, f_{N}$ do not themselves satisfy a nontrivial polynomial relation.

If $P=\left[a_{0}, \ldots, a_{N}\right] \in \mathbb{P}^{N}$, then we define $f(P)$ to be

$$
f(P):=\left[f_{0}\left(a_{0}, \ldots, a_{N}\right), \ldots, f_{N}\left(a_{0}, \ldots, a_{N}\right)\right]
$$

\footnotetext{
${ }^{2}$ Usually we will take $K$ to either have characteristic 0 or be a finite field, in which case $\bar{K}$ is an algebraic closure of $K$.
} 
provided at least one of the coordinates is nonzero. Note that if we use a different representative for $P$, say $P=\left[c a_{0}, \ldots, c a_{N}\right]$ for some $c \in \bar{K}^{*}$, then the coordinates of $f(P)$ are all multiplied by $c^{\operatorname{deg}(f)}$, so we get the same point in $\mathbb{P}^{N}$.

For $N=1$, a point $P \in \mathbb{P}^{1}$ is a critical point for the rational map $f: \mathbb{P}^{1} \rightarrow$ $\mathbb{P}^{1}$ if $f$ fails to be locally one-to-one in a neighborhood of $P$. Writing $f$ as a rational function $f(x) \in \bar{K}(x)$, the critical points of $f$ are the points where the derivative $f^{\prime}(x)$ vanishes, with some adjustment required if $P$ or $f(P)$ is the point at infinity. Maps whose critical points are all preperiodic are said to be postcritically finite, or PCF for short. The collection of PCF maps plays an important role in the study of one-dimensional dynamics.

Returning now to arbitrary $N \geq 1$, the indeterminacy locus of $f$, denoted $I(f)$, is the set of points at which $f$ is not well-defined, i.e.,

$$
I(f)=\left\{\left[a_{0}, \ldots, a_{N}\right] \in \mathbb{P}^{N}: f_{0}\left(a_{0}, \ldots, a_{N}\right)=\cdots=f_{N}\left(a_{0}, \ldots, a_{N}\right)=0\right\} .
$$

The map $f$ is said to be a morphism if $I(f)=\emptyset$, in which case $f$ is well-defined at every point of $\mathbb{P}^{N}$, and we write $f$ using a solid arrow $f: \mathbb{P}^{N} \rightarrow \mathbb{P}^{N}$. For $N=1$, it turns out that every rational map is a morphism, but this is no longer true for $N \geq 2$. We set the notation

$$
\operatorname{End}_{d}^{N}(K):=\left\{\begin{array}{l}
\text { morphisms } f: \mathbb{P}^{N} \rightarrow \mathbb{P}^{N} \text { of degree } d \text { given by } \\
\text { polynomials with coefficients in the field } K
\end{array}\right\} .
$$

The fact that a rational map $f: \mathbb{P}^{N} \rightarrow \mathbb{P}^{N}$ is not well-defined at every point is potentially a big problem if we want to study orbits of points under iteration of $f$. This prompts the following notation for the set of points having well-defined orbits:

$$
\mathbb{P}_{f}^{N}:=\left\{P \in \mathbb{P}^{N}: f^{n}(P) \notin I(f) \text { for all } n \geq 0\right\} .
$$

If we further want the coordinates of $P$ to be in the field $K$, we write $\mathbb{P}_{f}^{N}(K)$.

The zero set of a nonzero homogeneous polynomial $g \in \bar{K}\left[X_{0}, \ldots, X_{N}\right]$ is a welldefined subset of $\mathbb{P}^{N}$. The complement of $\{g=0\}$ is called a Zariski open subset of $\mathbb{P}^{N}$, and these form a base of open sets for the Zariski topology of $\mathbb{P}^{N}$. For our purposes, the most important concept is that of density for the Zariski topology.

Definition 3.2. A subset $S \subset \mathbb{P}^{N}$ is Zariski dense if

$$
\left\{g \in \bar{K}\left[X_{0}, \ldots, X_{N}\right]: g(P)=0 \text { for every } P \in S\right\}=\{0\},
$$

i.e., there are no nonzero homogeneous polynomials $g \in \bar{K}\left[X_{0}, \ldots, X_{N}\right]$ that vanish at every point of $S$.

Note to the reader. To make this article accessible to a wide audience, we have mostly restricted our attention to dynamics on projective space $\mathbb{P}^{N}$ over number fields, and frequently we restrict our attention to maps of $\mathbb{P}^{1}$ defined over $\mathbb{Q}$. Some sections conclude with a short subsection describing how the material generalizes to other algebraic varieties. In these addenda we have assumed that the reader is familiar with some basic algebraic geometry terminology and constructions, including, but not limited to, the following concepts.

A (projective) algebraic set $V$ is a subset of $\mathbb{P}^{N}$ defined by the set of solutions in $\mathbb{P}^{N}$ of a system of equations

$$
g_{1}\left(X_{0}, \ldots, X_{N}\right)=\cdots=g_{r}\left(X_{0}, \ldots, X_{N}\right)=0
$$


given by a collection of homogeneous polynomials $g_{1}, \ldots, g_{r} \in \bar{K}\left[X_{0}, \ldots, X_{N}\right]$. If $g_{1}, \ldots, g_{r}$ have coefficients in $K$, then we say that $V$ is defined over $K$, and then

$$
V(K)=V \cap \mathbb{P}^{N}(K) .
$$

A rational map $F: V \rightarrow V$ is the restriction to $V$ of a collection of rational maps $f_{1}, \ldots, f_{s}: \mathbb{P}^{N} \rightarrow \mathbb{P}^{N}$ having the property that

$$
f_{i}\left(V \backslash I\left(f_{i}\right)\right) \subseteq V \quad \text { and } \quad f_{i}(P)=f_{j}(P) \text { for all } P \in V \backslash\left(I\left(f_{i}\right) \cup I\left(f_{j}\right)\right) .
$$

In other words, we create $F$ in the standard way by gluing the $f_{i}$ along the subsets of $V$ on which they agree. The map $F$ is well-defined at all points of $V$ outside the indeterminacy locus $I(F):=\bigcap_{i=1}^{s} I\left(f_{i}\right)$. Thus if $P \notin I\left(f_{j}\right)$ for some $j$, then $F(P)=f_{j}(P)$. If $I(F)=\emptyset$, then we say that $F$ is a morphism, or an endomorphism of $V$.

\section{UNIFORM BOUNDEDNESS OF (PRE)PERIODIC POINTS}

A rational function $f(x) \in K(x)$ induces a map on the $K$-rational points of the projective line

$$
f: \mathbb{P}^{1}(K) \longrightarrow \mathbb{P}^{1}(K) .
$$

When $K=\mathbb{R}$ or $K=\mathbb{C}$, the distribution of the (pre)periodic points is intimately connected to the dynamics of $f$. For fields of arithmetic interest, there is a famous, frequently rediscovered, finiteness theorem of Northcott.

Theorem 4.1 (Northcott, 1950, [181]). Let $N \geq 1$ and $d \geq 2$, and let $K / \mathbb{Q}$ be a number field. Then for all degree $d$ morphisms $f: \mathbb{P}^{N} \rightarrow \mathbb{P}^{N}$ defined over $K$, the set of $K$-rational preperiodic points

$$
\operatorname{PrePer}\left(f, \mathbb{P}^{N}(K)\right) \text { is finite. }
$$

In view of Northcott's theorem, it is natural to inquire as to the possible size of the finite set $\operatorname{PrePer}\left(f, \mathbb{P}^{N}(K)\right)$, which leads to the following fundamental conjecture.

Conjecture 4.2 (Dynamical Uniform Boundedness Conjecture: Morton-Silverman, 1994, 179). Fix integers $N \geq 1, d \geq 2$, and $D \geq 1$. There is a constant $C(N, d, D)$ such that for all degree $d$ morphisms $f: \mathbb{P}^{N} \rightarrow \mathbb{P}^{N}$ defined over a number field $K$ of degree $[K: \mathbb{Q}]=D$, the number of $K$-rational preperiodic points is uniformly bounded,

$$
\text { \# PrePer }\left(f, \mathbb{P}^{N}(K)\right) \leq C(N, d, D) .
$$

Remark 4.3. Fakhruddin 65] has shown that Conjecture 4.2, which is a uniform boundedness conjecture for projective space, implies an analogous folklore uniform boundedness conjecture for torsion points on abelian varieties. This latter conjecture generalizes theorems for elliptic curves due to Mazur [170, Kamienny 133, and Merel [172].

Very little is known about this conjecture, although there are weaker bounds in which the constant $C(N, d, D)$ is replaced by a constant that depends in various weak ways on the number of primes of bad reduction of $f$; see for example 27,31, as well as the discussion in Section[12. Indeed, even for the family of polynomials $x^{2}+c$, we have only fragmentary knowledge, as in the following result. 
Theorem 4.4. For $c \in \mathbb{Q}$, let $f_{c}(x)=x^{2}+c$. Let $N \geq 1$.

(a) For $N \in\{1,2,3\}$, there are infinitely many $c \in \mathbb{Q}$ such that $f_{c}$ has a periodic point $\alpha \in \mathbb{Q}$ of exact period $N$.

(b) (Morton, 1992, 177]; Flynn-Poonen-Schaefer, 1997, 81]; Stoll, 2008, 228]) For $N \in\{4,5,6\}$, there are no values of $c \in \mathbb{Q}$ such that $f_{c}$ has a periodic point $\alpha \in \mathbb{Q}$ of exact period $N$, with the caveat that the proof for $N=6$ is conditional on the conjecture of Birch and Swinnerton-Dyer.

The proof of Theorem 4.4(b) is geometric and global in nature. See [140] for a local proof for $N=4$ and, with an additional assumption, $N=5$.

Conjecture 4.5 (Flynn-Poonen-Schaefer, 1997, [81]). For all $c \in \mathbb{Q}$, the set of periodic points $\operatorname{Per}\left(x^{2}+c, \mathbb{Q}\right)$ contains no points of period strictly greater than 3 .

Theorem 4.6 (Poonen, 1998, 191). If Conjecture 4.5 is true, then

$$
\sup _{c \in \mathbb{Q}} \# \operatorname{PrePer}\left(x^{2}+c, \mathbb{P}^{1}(\mathbb{Q})\right)=9 \text {, }
$$

with the maximum occurring for infinitely many values of c, including for example $c=-\frac{29}{16}$ and $c=-\frac{21}{16}$. (All possible preperiodic structures are described in [191].)

There are many other interesting families of rational maps that have been studied. For example, rational maps of degree 2 that satisfy $f(-x)=-f(x)$ form the one-dimensional family ${ }^{3}$ of maps $f(x)=b x+c x^{-1}$.

Conjecture 4.7 (Manes, 2008, [161]). A $\mathbb{Q}$-rational periodic point of a map of the form $b x+c x^{-1}$ with $b, c \in \mathbb{Q}^{*}$ has period 1,2 , or 4 . Further,

$$
\sup _{b, c \in \mathbb{Q}^{*}} \# \operatorname{PrePer}\left(b x+c x^{-1}, \mathbb{P}^{1}(\mathbb{Q})\right)=12 .
$$

More generally, we may consider the two-dimensional family consisting of all degree 2 rational maps of $\mathbb{P}^{1}$ defined over $\mathbb{Q}$.

Conjecture 4.8 (Benedetto-Chen-Hyde-Kovacheva-White, 2014, [34; BlancCanci-Elkies, 2015, 39]). Let $f \in \operatorname{End}_{2}^{1}(\mathbb{Q})$ be a rational map of degree 2 defined over $\mathbb{Q}$.

(a) Let $P \in \mathbb{P}^{1}(\mathbb{Q})$ be periodic for $f$. Then $P$ has period at most 7 .

(b) Let $P \in \mathbb{P}^{1}(\mathbb{Q})$ be preperiodic for $f$. Then $\# \mathcal{O}_{f}(P) \leq 9$.

(c) The full set of rational preperiodic points satisfies \# $\operatorname{PrePer}\left(f, \mathbb{P}^{1}(\mathbb{Q})\right) \leq 14$.

We note that there are examples in [34,39] showing that the values 7, 9, and 14 in Conjecture 4.8 are optimal.

Generalization to algebraic varieties. Poonen has asked whether a uniform bound, analogous to Conjecture 4.2, is true for preperiodic points in arbitrary families of maps. We restate a version of Poonen's question as a conjecture.

Conjecture 4.9 (Poonen, 2012, [192, Question 3.2.]). Let $K$ be a number field, let $X$ and $S$ be varieties defined over $K$, let $\pi: X \rightarrow S$ be a morphism, and let $f$ : $X \rightarrow X$ be an $S$-morphism, which means that $\pi \circ f=\pi$. For each point $s \in S(\bar{K})$,

\footnotetext{
${ }^{3}$ Despite the two parameters $b$ and $c$ appearing in $b x+c x^{-1}$, geometrically this is actually a one-dimensional family because, over an algebraically closed field, the change of variable $c^{-1 / 2} f\left(c^{1 / 2} x\right)=b x+x^{-1}$ eliminates $c$. In general, we call $c$ a twist parameter since the dynamical properties of the map depend only on $c$ modulo squares.
} 
the map $f$ restricted to the fiber $X_{s}:=\pi^{-1}(s)$ gives a morphism $f_{s}: X_{s} \rightarrow X_{s}$, and we view $f: X \rightarrow X$ as a family of maps parameterized by the points of $S$. Then for all $D \geq 1$, we have

$$
\sup _{\substack{L / K \\[L: K]=D}} \sup _{s \in S(L)} \# \operatorname{PrePer}\left(f_{s}, X_{s}(L)\right)<\infty .
$$

Taking $X \rightarrow S$ to be the universal family of degree $d$ endomorphisms of $\mathbb{P}^{N}$ (cf. Section 10), one sees that Conjecture 4.9 implies the Uniform Boundedness Conjecture (Conjecture 4.2).

\section{Arboreal representations}

Let $K$ be a field of characteristic 0 , let $\bar{K}$ be an algebraic closure of $K$, and let $f: \mathbb{P}^{N} \rightarrow \mathbb{P}^{N}$ be a morphism of degree $d \geq 2$ defined over $K 4$ Rather than looking at forward orbits of points, in this section we start with an initial point $P \in \mathbb{P}^{N}(K)$, and we look at the points in its backward orbit,

$$
\mathcal{O}_{f}^{-}(P):=\left\{Q \in \mathbb{P}^{N}(\bar{K}): f^{k}(Q)=P \text { for some } k \geq 0\right\} .
$$

Writing

$$
f^{-n}(P):=\left\{Q \in \mathbb{P}^{N}(\bar{K}): f^{n}(Q)=P\right\},
$$

we see that the disjoint union

$$
T_{f, \infty}(P):=\{P\} \sqcup f^{-1}(P) \sqcup f^{-2}(P) \sqcup f^{-3}(P) \sqcup \cdots
$$

acquires the structure of a rooted tree, with root vertex $P$, where we assign edges according to the action of $f$.

Definition 5.1. We say that the tree $T_{f, \infty}(P)$ is complete if $\# f^{-n}(P)=d^{n}$ for every $n \geq 0$, i.e., if $T_{f, \infty}(P)$ is a complete rooted $d$-ary tree.

For example, if $N=1$, then $T_{f, \infty}(P)$ is complete if and only if the inverse image $f^{-n}(P)$ contains no critical points of $f$ for all $n \geq 1$. Figure 1illustrates a complete inverse image tree for a degree 2 map $f: \mathbb{P}^{1} \rightarrow \mathbb{P}^{1}$.

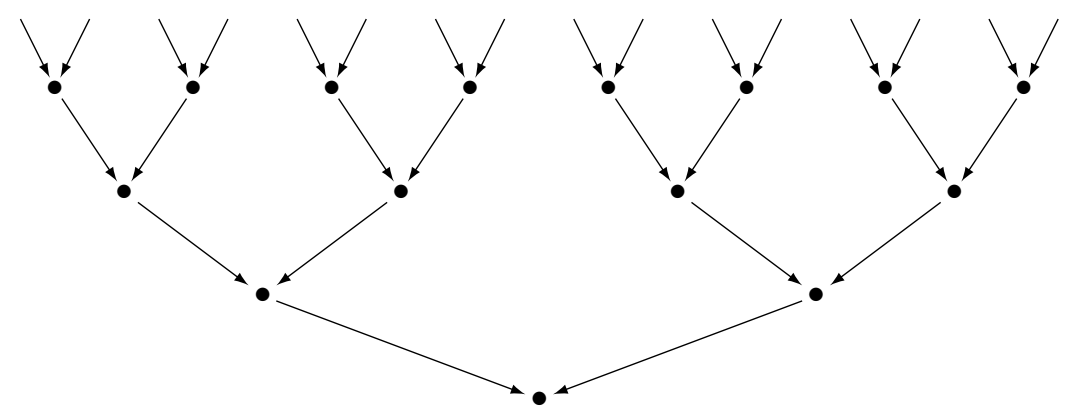

Figure 1. A complete binary inverse image tree

\footnotetext{
${ }^{4}$ More generally, we could take $f: V \rightarrow V$ to be a self-morphism of a quasi-projective variety, for example a polynomial map $f: \mathbb{A}^{N} \rightarrow \mathbb{A}^{N}$, subject to suitable assumptions.
} 
The coordinates of the points in $\mathcal{O}_{f}^{-}(P)$ generate finite extension fields of $K$. For each $n \geq 1$, we let

$K_{f, n}(P):=$ the extension of $K$ generated by the coordinates of points in $f^{-n}(P)$, and we let

$$
K_{f, \infty}(P):=\bigcup_{n \geq 0} K_{f, n}(P)
$$

be the field generated by the coordinates of all of the points in the full backward orbit of $P$. In the case that $K$ is a number field, we can use the theory of height functions as described in Section 14 to show that $T_{f, \infty}(P)$ is a set of points of bounded height, which in turn implies that $\left[K_{f, \infty}(P): K\right]=\infty$ provided that the tree has infinitely many distinct points.

For the rest of this section, we make the following assumption:

$$
\text { The tree } T_{f, \infty}(P) \text { is complete. }
$$

Then the Galois group $G_{K}:=\operatorname{Gal}(\bar{K} / K)$ acts on $f^{-n}(P)$ through its action on the coordinates of each point. Moreover, this action commutes with $f$, since $f$ is defined over $K$. We let 5

$$
\operatorname{Aut}\left(T_{f, \infty}(P)\right)=\text { the group of tree automorphisms of } T_{f, \infty}(P) \text {. }
$$

The action of $G_{K}$ on the points in $T_{f, \infty}(P)$ yields a homomorphism

$$
\rho_{f, P}^{\mathrm{arb}}: G_{K} \rightarrow \operatorname{Aut}\left(T_{f, \infty}(P)\right) \text {. }
$$

The map $\rho_{f . P}^{\text {arb }}$ is called the arboreal representation associated to the map $f$ and basepoint $P[6$ and we denote its image by

$$
G_{f, \infty}(P):=\rho_{f, P}^{\mathrm{arb}}\left(G_{K}\right) \cong \operatorname{Gal}\left(K_{f, \infty}(P) / K\right) .
$$

One can also realize $G_{f, \infty}(P)$ by taking the inverse limit of the groups $G_{f, n}(P):=$ $\operatorname{Gal}\left(K_{f, n}(P) / K\right)$ with respect to the natural restriction maps.

Characterizing the size of the image of $\rho_{f, P}^{\text {arb }}$ is one of the main problems in this area, inspired in part by finite-index results for Galois representations associated to elliptic curves due to Serre [204,205] and analogous conjectures for abelian varieties.

Definition 5.2. The Odoni index of the map $f$ and basepoint $P$ over the field $K$ is the quantity

$$
\iota_{K}(f, P):=\left[\operatorname{Aut}\left(T_{f, \infty}(P)\right): G_{f, \infty}(P)\right] .
$$

\section{Question 5.3.}

(a) For which choices of fields $K$, maps $f$, and basepoints $P$ is the Odoni index $\iota_{K}(f, P)$ equal to 1 , i.e., when is the arboreal representation $\rho_{f, P}^{\text {arb }}$ surjective?

(b) For which choices of fields $K$, maps $f$, and basepoints $P$ is the Odoni index $\iota_{K}(f, P)$ finite, i.e., when does the image of the arboreal representation $\rho_{f, P}^{\text {arb }}$ have finite index in the full tree automorphism group?

\footnotetext{
${ }^{5}$ We remark that the automorphism group of an $n$-level $d$-branched tree may be identified with the $n$-fold wreath product of the symmetric product $\mathcal{S}_{d}$, so $\operatorname{Aut}\left(T_{\infty}(P)\right)$ is isomorphic to the inverse limit of $\mathcal{S}_{d}$ 々 $\mathcal{S}_{d} \prec \cdots<\mathcal{S}_{d}$ ( $n$ copies) as $n \rightarrow \infty$.

${ }^{6}$ The term "arboreal representation" for homomorphisms of Galois groups to automorphism groups of trees was coined by Nigel Boston around 2003.
} 
Virtually nothing is known for $N \geq 2$, so we restrict our attention to maps $f: \mathbb{P}^{1} \rightarrow \mathbb{P}^{1}$. In this case, Odoni 182 showed in 1985 that if the coefficients of a monic polynomial $f(X)$ are independent indeterminates, then $\iota_{K}(f, 0)=1$, and in $[183]$ he also gave the explicit example $\iota_{\mathbb{Q}}\left(X^{2}-X+1,0\right)=1$.

Conjecture 5.4 (Odoni, 1985, 182). Let $K$ be a Hilbertian field 7 and let $d \geq 2$. There exists a monic polynomial $f(X) \in K[X]$ of degree $d$ and a point $P \in K$ such that $\iota_{K}(f, P)=1$. (Addendum [125, Jones, 2013]: If $K$ is a number field, then one can take the coefficients of $f(X)$ to lie in the ring of integers of $K$.)

Looper 157 recently proved Odoni's conjecture (Conjecture 5.4) for $K=\mathbb{Q}$ and all prime degrees $d$, and a number of researchers [23, 132, 226] independently extended Looper's work. In particular, Specter [226] proved Odoni's conjecture for all number fields, and more generally, for all algebraic extensions $K / \mathbb{Q}$ that are unramified outside of a finite set of primes.

We note that for any $f$ defined over $K$, there is always a finite extension field $L / K$ such that $\iota_{L}(f, P) \geq 2$. For example, we can simply adjoin a point in $f^{-1}(P)$ to $K$. So we turn our attention to the fundamental question of when $\iota_{K}(f, P)$ is finite.

Over number fields $K$, it is known that the Odoni index $\iota_{K}(f, P)$ is finite for certain families of quadratic polynomials [123, 227, as well as for various quadratic rational functions [128] and higher-degree polynomials [157, and there are examples over global function fields [111. See [125] for a survey of known results.

On the other hand, there are several conditions that are known to ensure that $\iota_{K}(f, P)=\infty$. These include the map being PCF 8 and $P$ arbitrary, maps for which there is a $K$-automorphism of $\mathbb{P}^{1}$ commuting with $f$ and fixing $P$, using a basepoint $P$ that is a periodic point of $f$, and choosing a map $f$ such that the forward orbits of its critical points intersect in certain ways. In the case $N=1$ and $d=2$, we now have enough knowledge to plausibly conjecture that these are the only exceptions to finite Odoni index.

Conjecture 5.5 ([125, Conjecture 3.11]). Let $K$ be a field of characteristic 0, and suppose that $f(x) \in K(x)$ is a rational function of degree 2 . Then

$$
\iota_{K}(f, P)=\infty
$$

if and only if one of the following holds.

(a) The map $f$ is PCF, i.e., the critical points of $f$ are preperiodic.

(b) There is an $r \geq 2$ so that the two critical points $\gamma_{1}$ and $\gamma_{2}$ of $f$ satisfy a relation of the form $f^{r}\left(\gamma_{1}\right)=f^{r}\left(\gamma_{2}\right)$.

(c) The basepoint $P$ of the tree $T_{f, \infty}(P)$ is periodic under $f$.

(d) There is a nontrivial Möbius transformation $\varphi(x)=\frac{\alpha x+\beta}{\gamma x+\delta}$ defined over $K$ that satisfie?

$$
\varphi \circ f(x)=f \circ \varphi(x) \quad \text { and } \quad \varphi(P)=P .
$$

${ }^{7}$ A field $K$ is Hilbertian if it is not possible to cover $\mathbb{P}^{1}(K)$ by a union of finitely many sets of the form $F(C(K))$, where $C / K$ is a smooth curve and $F: C \rightarrow \mathbb{P}^{1}$ is a finite map of degree at least 2. For example, all number fields are Hilbertian, but $\mathbb{R}, \mathbb{C}$, and $\mathbb{Q}_{p}$ are not.

${ }^{8}$ We recall that a map is post-critically finite $(\mathrm{PCF})$ if its critical points are preperiodic.

${ }^{9}$ See Section 10 for a detailed discussion of Aut $(f)$, which is the group of automorphisms of $\mathbb{P}^{N}$ that commute with $f$. 

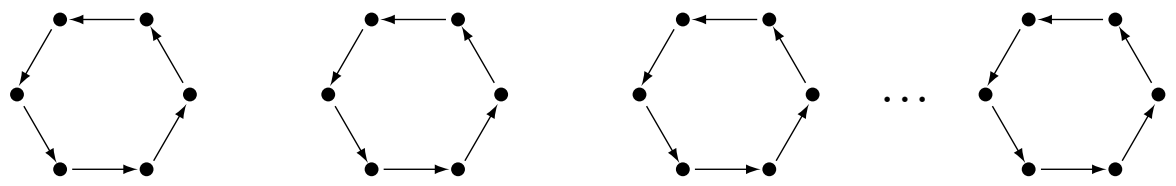

Figure 2. A set of points of period 6

\section{Dynatomic Representations}

Let $K$ be a field of characteristic 0 with algebraic closure $\bar{K}$, and let $f: \mathbb{P}^{N} \rightarrow \mathbb{P}^{N}$ be a morphism of degree $d \geq 2$ defined over $K$. We recall that for each $n \geq 1$, we write

$$
\operatorname{Per}_{n}^{*}\left(f, \mathbb{P}^{N}(\bar{K})\right):=\left\{P \in \mathbb{P}^{N}(\bar{K}): f^{n}(P)=P \text { and } f^{k}(P) \neq P \text { for } 1 \leq k<n\right\}
$$

for the set of points of exact period $n$. We note that $f$ maps this set to itself, and that the resulting graph can be represented as a union of $n$-gons. Figure 2 illustrates $\operatorname{Per}_{6}^{*}(f)$, which is a collection of periodic cycles of period 6 .

An automorphism of a collection of $n$-cycles consists of a permutation of the cycles and a rotation of each cycle. For $\nu \geq 1$, we set the notation

$$
\mathcal{P}_{n, \nu}^{*}:=\text { a disjoint union of } \nu \text { periodic } n \text {-cycles. }
$$

Then the automorphism group of $\mathcal{P}_{n, \nu}^{*}$ is most easily described as a wreath product, which by definition is a semidirect product,

$$
\operatorname{Aut}\left(\mathcal{P}_{n, \nu}^{*}\right) \cong(\mathbb{Z} / n \mathbb{Z})<\mathcal{S}_{\nu}:=(\mathbb{Z} / n \mathbb{Z})^{\nu} \rtimes \mathcal{S}_{\nu} .
$$

The group law on this semidirect product is given by

$$
\left(\left(c_{1}, \ldots, c_{\nu}\right), \pi\right) \cdot\left(\left(c_{1}^{\prime}, \ldots, c_{\nu}^{\prime}\right), \pi^{\prime}\right):=\left(\left(c_{1}+c_{\pi(1)}^{\prime}, \ldots, c_{\nu}+c_{\pi(\nu)}^{\prime}\right), \pi \pi^{\prime}\right),
$$

where $c_{i}$ describes a rotation of the $i$ th cycle and $\pi$ permutes the cycles. In particular, the automorphism group of $\mathcal{P}_{n, \nu}^{*}$ has order \# $\operatorname{Aut}\left(\mathcal{P}_{n, \nu}^{*}\right)=n^{\nu} \cdot \nu$ !.

The equation $f^{n}(P)=P$ is equivalent to the intersection of $N$ hypersurfaces of degree $d^{n}+1$ in $\mathbb{P}^{N}$, so by Bézout's theorem it consists of $(d+1)^{N}$ points, counted with multiplicities. If all of the points have multiplicity 1 , then the number of points in $\operatorname{Per}_{n}^{*}(f)$ is given by an inclusion/exclusion formula. One expects this to be the case for sufficiently large $n$, as in the following conjecture 10

Conjecture 6.1. Let $K$ be a field of characteristic 0 , and let $f: \mathbb{P}^{N} \rightarrow \mathbb{P}^{N}$ be a morphism of degree $d \geq 2$ defined over $K$. Then for all sufficiently large $n$ we have11

$$
\# \operatorname{Per}_{n}^{*}\left(f, \mathbb{P}^{N}(\bar{K})\right):=\sum_{m \mid n} \mu\left(\frac{n}{m}\right)\left(d^{m}+1\right)^{N} .
$$

We note that Conjecture 6.1 is true for $N=1$. This follows from the fact that there are only finitely many rationally indifferent periodic points, i.e., periodic points whose multiplier is a root of unity. This in turn is a consequence of a theorem

\footnotetext{
${ }^{10}$ Let $P \in \operatorname{Per}_{n}^{*}(f)$. Then $P$ is fixed by $f^{n}$, so $f^{n}$ induces a linear map $D f^{n}(P)$ on the tangent space of $\mathbb{P}^{N}$ at $P$, and the periodic point multiplicity of $P$ is greater than 1 if and only if $D f^{n}(P)$ has an eigenvalue equal to 1 . For example, the point $\frac{1}{2}$ is a fixed point of multiplicity 2 for the map $f(x)=x^{2}+\frac{1}{4}$, since $f(x)-x=\left(x-\frac{1}{2}\right)^{2}$, and we observe that $f^{\prime}\left(\frac{1}{2}\right)=1$.

${ }^{11}$ The function $\mu$ is the Möbius function, which we recall is defined as follows: If $n$ is a product of $k$ distinct primes, then $\mu(n)=(-1)^{k}$; otherwise, $\mu(n)=0$.
} 
of Fatou that a rational function $f(x) \in \mathbb{C}(x)$ of degree $d \geq 2$ has at most $6 d-6$ nonrepelling periodic cycles [175, Theorem 13.1], a bound that was improved to the best possible $2 d-2$ by Shishikura [207.

Since we have assumed that $f$ is defined over $K$, we see that the action of the Galois group $G_{K}:=\operatorname{Gal}(\bar{K} / K)$ commutes with $f$, i.e.,

$$
\sigma(f(P))=f(\sigma(P)) \quad \text { for all } P \in \mathbb{P}^{N}(\bar{K}) \text { and all } \sigma \in G_{K} .
$$

It follows that $G_{K}$ acts on the set $\operatorname{Per}_{n}^{*}\left(f, \mathbb{P}^{N}(\bar{K})\right)$, and that this action preserves the cycle structure induced by $f$. We let $\nu=\nu(n, f):=n^{-1} \# \operatorname{Per}_{n}^{*}\left(f, \mathbb{P}^{N}(\bar{K})\right)$, which allows us to identify $\operatorname{Per}_{n}^{*}\left(f, \mathbb{P}^{N}(\bar{K})\right)$ with $\mathcal{P}_{n, \nu}^{*}$. Then the action of $G_{K}$ on $\operatorname{Per}_{n}^{*}\left(f, \mathbb{P}^{N}(\bar{K})\right)$ yields a homomorphism

$$
\rho_{n, f}^{\mathrm{dyn}}: G_{K} \longrightarrow \operatorname{Aut}\left(\mathcal{P}_{n, \nu}^{*}\right) .
$$

The map $\rho_{n, f}^{\mathrm{dyn}}$ is called the $n$-level dynatomic representation associated to $f$. It is a fundamental problem to determine the size of its image. For example, does the image have index bounded independent of $n$ ? And is $\rho_{n, f}^{\mathrm{dyn}}$ surjective for all sufficiently large $n$ ?

An issue regarding this last question arises if the map $f$ admits automorphisms that are defined over $K$. We define 12

$$
\operatorname{Aut}_{K}(f):=\left\{\varphi \in \mathrm{PGL}_{N+1}(K): \varphi \circ f=f \circ \varphi\right\} .
$$

Elements of $\operatorname{Aut}_{K}(f)$ act on $\operatorname{Per}_{n}^{*}\left(f, \mathbb{P}^{N}(\bar{K})\right)$ and commute with both $f$ and the action of $G_{K}$. This puts a further constraint on the image of $\rho_{n, f}^{\text {dyn }}$, which leads us to define

$$
\operatorname{Aut}\left(\mathcal{P}_{n, \nu}^{*}\right)^{\operatorname{Aut}_{K}(f)}:=\left\{\alpha \in \operatorname{Aut}\left(\mathcal{P}_{n, \nu}^{*}\right): \varphi \circ \alpha=\alpha \circ \varphi \text { for all } \alpha \in \operatorname{Aut}_{K}(f)\right\} .
$$

Question 6.2. Let $K$ be a field of characteristic 0 , let $f: \mathbb{P}^{N} \rightarrow \mathbb{P}^{N}$ be a morphism of degree $d \geq 2$ defined over $K$, and for $n \geq 1$ let $\nu=\nu(n, f)=$ $n^{-1} \# \operatorname{Per}_{n}^{*}\left(f, \mathbb{P}^{N}(\bar{K})\right)$ and fix an identification $\operatorname{Per}_{n}^{*}\left(f, \mathbb{P}^{N}(\bar{K})\right) \cong \mathcal{P}_{n, \nu}^{*}$. Find general necessary and sufficient conditions on $f$ for the following statements to hold:

(a) The index of the dynatomic representation $\rho_{n, f}^{\mathrm{dyn}}$ is bounded, i.e.,

$$
\sup _{n \geq 1}\left(\operatorname{Aut}\left(\mathcal{P}_{n, \nu}^{*}\right): \rho_{n, f}^{\text {dyn }}\left(G_{K}\right)\right)<\infty .
$$

(b) For all sufficiently large $n$, the dynatomic representation $\rho_{n, f}^{\mathrm{dyn}}$ is as surjective as possible, i.e.,

$$
\rho_{n, f}^{\mathrm{dyn}}\left(G_{K}\right)=\operatorname{Aut}\left(\mathcal{P}_{n, \nu}^{*}\right)^{\operatorname{Aut}_{K}(f)} .
$$

Very little is known about Question 6.2, although it is not hard to find $f$ for which the assertions are probably false.

Example 6.3. Let $p=2^{\ell}-1$ be a Mersenne prime. Then $\operatorname{Per}_{\ell}^{*}\left(x^{2}\right)$ is the set of primitive $p$ th roots of unity $\boldsymbol{\mu}_{p}^{*}$, which the map $x \rightarrow x^{2}$ separates into $(p-1) / \ell$ distinct $\ell$-cycles. The action of $\operatorname{Gal}(\overline{\mathbb{Q}} / \mathbb{Q})$ on $\boldsymbol{\mu}_{p}^{*}$ is completely determined by where

\footnotetext{
${ }^{12}$ See Section 10 for a detailed discussion of $\operatorname{Aut}(f)$.
} 
it sends a single element of $\boldsymbol{\mu}_{p}^{*}$, so the Galois action cannot come close to being surjective. More precisely, we have

$$
\begin{gathered}
\# \rho_{\ell, x^{2}}^{\mathrm{dyn}}\left(G_{\mathbb{Q}}\right)=p-1, \quad \operatorname{Aut}_{\mathbb{Q}}(f)=\left\{x, x^{-1}\right\}, \\
\# \operatorname{Aut}\left(\mathcal{P}_{\ell,(p-1) / \ell}^{*}\right)^{\operatorname{Aut}_{\mathbb{Q}}(f)}=\ell^{(p-1) / \ell} \cdot(p-1) / \ell ! .
\end{gathered}
$$

\section{INTERSECTIONS OF ORBITS AND SUBVARIETIES}

Let $X$ be an algebraic variety, let $f: X \rightarrow X$ be a morphism, let $Y \subseteq X$ be a subvariety of $X$, and let $P \in X$ be a wandering point for $f$. It is natural to ask under what circumstances the orbit $\mathcal{O}_{f}(P)$ intersects the subvariety $Y$ at infinitely many points. For example, one way that this can happen is if $Y$ contains a subvariety $Z \subset Y$ of dimension at least 1 having the property that $f^{n}(Z) \subset Z$ for some integer $n \geq 1$ and there is some $m \geq 0$ such that $f^{m}(P) \in Z$, since then $f^{n k+m}(P) \in Z \subset Y$ for all $k \geq 0$.

In order to state a precise conjecture, we define a (one-sided) arithmetic progression in $\mathbb{N}$ to be a collection of integers of the form

$$
\{a k+b: k=0,1,2, \ldots\}
$$

for some fixed integers $a \geq 0$ and $b \geq 0$. Note that we permit $a=0$, so a singleelement set $\{b\}$ is an arithmetic progression.

Conjecture 7.1 (Dynamical Mordell-Lang Conjecture: Bell, 2006, [17]; Denis, 1994, 56]; Ghioca-Tucker, 2009, 86]). Let X/C be a quasi-projective variety, let $f: X \rightarrow X$ be a morphism, let $P \in X$ be a point, and let $Y \subseteq X$ be a subvariety of $X$. Then

$$
\left\{n \geq 0: f^{n}(P) \in Y\right\}
$$

is a finite union of arithmetic progressions.

Before stating a consequence of the conjecture, we generalize the notion of (pre)periodic point to (pre)periodic subvariety.

Definition 7.2. Let $f: X \rightarrow X$ be an endomorphism of a quasi-projective variety. A subvariety $Y \subset X$ is periodic if $f^{n}(Y) \subseteq Y$ for some $n \geq 1$, and $Y$ is preperiodic if $f^{n}(Y) \subseteq f^{m}(Y)$ for some $n>m \geq 0$.

Conjecture 7.1 implies that if $P$ is wandering and $\mathcal{O}_{f}(P) \cap Y$ is infinite, then the Zariski closure of $\mathcal{O}_{f}(P) \cap Y$ contains a periodic subvariety $Z$ of dimension at least 1. In particular, if $Y$ contains no $f$-periodic subvarieties of dimension at least 1 , then conjecturally the intersection $\mathcal{O}_{f}(P) \cap Y$ is finite.

There is an extensive literature proving various special cases of Conjecture 7.1 see for example [18, 36, 45, 85, 88, 93, 95, 147, 186, 223, 239, 241, 243. Here are two notable examples.

Theorem 7.3. Conjecture 7.1 is true in the following cases.

(a) (Xie, 2015, 241]) The map $f: \mathbb{A}^{2} \rightarrow \mathbb{A}^{2}$ is a morphism defined over $\overline{\mathbb{Q}}$.

(b) (Bell-Ghioca-Tucker, 2010, [15]) The map $f: X \rightarrow X$ is unramified. Note that this includes the case that $f$ is an automorphism, e.g., a polynomial automorphism of $\mathbb{A}^{N}$.

There are also variants of Conjecture 7.1 for monoids generated by more than one morphism, as in the following result. 
Theorem 7.4 (Ghioca-Tucker-Zieve, 2016, 18]). Let $f_{1}(x), f_{2}(x) \in \mathbb{C}[x]$ be polynomials of degree greater than 1 . Let $\alpha \in \mathbb{C}$ be a wandering point of $f_{1}$, and let $\beta \in \mathbb{C}$ be a wandering point of $f_{2}$. Suppose that there are infinitely many pairs $\left(n_{1}, n_{2}\right) \in \mathbb{N}^{2}$ such that $f_{1}^{n_{1}}(\alpha)=f_{2}^{n_{2}}(\beta)$. Then there are positive integers $\ell_{1}$ and $\ell_{2}$ such that $f_{1}^{\ell_{1}}(x)=f_{2}^{\ell_{2}}(x)$.

On the other hand, if one takes finitely many commuting morphisms

$$
f_{1}, \ldots, f_{g}: X \longrightarrow X
$$

then it is impossible to say much of anything about the structure of the set

$$
\left\{\left(n_{1}, \ldots, n_{g}\right) \in \mathbb{N}^{g}: f_{1}^{n_{1}} \circ \cdots \circ f_{g}^{n_{g}}(P) \in Y\right\} .
$$

Indeed, Scanlon and Yasufuku [201] (see also 93]) have shown that the set (7.1) can be arbitrarily complicated in a way that can be made precise using the language of polynomial-exponential equations.

Conjecture 7.1 says that if a point $P$ is not contained in a proper $f$-preperiodic subvariety, then the intersection $\mathcal{O}_{f}(P) \cap Y$ with any subvariety $Y \subsetneq X$ is finite. This raises the question of whether there exist any algebraic points whose orbit does not lie in a proper subvariety, i.e., whose orbit is Zariski dense. An obvious obstruction to the existence of such points would be a fibering as in the following definition.

Definition 7.5. Let $X$ be a quasi-projective variety, and let $f: X \rightarrow X$ be an endomorphism. We say that $f$ is a fibered map if there exists a positive dimensional variety $Z$ and a dominant rational map $\psi: X \rightarrow Z$ such that $\psi \circ f=\psi$.

The following general conjecture, which proposes that fibering is the only possible obstruction, builds on an earlier conjecture due to Zhang [246].

Conjecture 7.6 (Medvedev-Scanlon, 2009, [171, Conjecture 5.10]; Amerik-Bogomolov-Rovinsky, 2011, 2, Conjecture 1.2]). Let $X$ be a quasi-projective variety defined over an algebraically closed field $K$ of characteristic 0 . Let $f: X \rightarrow X$ be an endomorphism defined over $K$, and assume that $f$ is not a fibered map.

(a) Weak form: There exists a point $P \in X(K)$ whose orbit $\mathcal{O}_{f}(P)$ is Zariski dense in $X$.

(b) Strong form: There is a Zariski dense set of points $\mathcal{Z} \subset X(K)$ having disjoint orbits such that $\mathcal{O}_{f}(P)$ is Zariski dense for every $P \in \mathcal{Z}$.

Conjecture 7.6. (a) has been proven in a handful of cases [2, 3, 16, 171, including, in particular, that Xie 244 has shown it is true for $X=\mathbb{A}^{2}$. But the general case remains very much open, even in the case that $f$ is an automorphism.

Algebraically closed fields such as $\overline{\mathbb{Q}}$ are interesting but, especially when working with projective space, one often wants points defined over a specific number field or over fields of bounded degree.

Question 7.7. Is Conjecture [7.6 true for $X=\mathbb{A}^{N}$ if $K$ is a number field? More generally, if $K$ is a number field, is there a constant $C=C(X / K)$ so that Conjecture 7.6 true if we restrict to points $P \in X(\bar{K})$ satisfying $[K(P): K] \leq C$ ?

Remark 7.8. The classical Mordell-Lang conjecture was proven by Faltings 67, 68, and the rank 1 case fits into the setup of this section. Let $A / \mathbb{C}$ be an abelian variety, let $Q \in A(\mathbb{C})$ be a point, and let $T_{Q}: A \rightarrow A$ be the translation-by- $Q$ map defined 
by $T_{Q}(P)=P+Q$. Let $Y \subseteq A$ be a subvariety of $A$. Then Faltings' theorem implies that Conjecture 7.1 is true for the map $T_{Q}$, i.e., the set of $n \geq 0$ such that $T_{Q}^{n}(P)=n P+Q \in Y$ is a finite union of arithmetic progressions. The general case of the classical Mordell-Lang conjecture has a similar formulation using the monoid of maps generated by a finite list $T_{Q_{1}}, \ldots, T_{Q_{r}}$ of translations 13

For a general dominant rational map $f: X \rightarrow X$, there may not be a subvariety $X_{0} \subset X$ on which $f$ induces a morphism $f: X_{0} \rightarrow X_{0}$, so a first question is whether there are any points having well-defined forward orbits.

We recall that $X_{f}(K)$ denotes the set of points $P \in X(K)$ for which the entire forward orbit $\mathcal{O}_{f}(P)$ is well-defined. Working over $\mathbb{C}$, it is clear that the wandering points in $X_{f}(\mathbb{C})$ are Zariski dense, since the the complement $\bigcup_{n>0} f^{-n}(I(f))$ is a countable union of proper subvarieties of $X$. If we replace $\mathbb{C}$ with $\overline{\mathbb{Q}}$, this statement becomes much harder to prove, since $X(\overline{\mathbb{Q}})$ itself is countable. Indeed, it is not even clear, a priori, that $X_{f}(\overline{\mathbb{Q}})$ is nonempty, especially if we restrict our attention to wandering points.

Theorem 7.9 (Amerik, 2011, 3]). Let $X$ be a quasi-projective variety defined over an algebraically closed field $K$ of characteristic 0 , and let $f: X \rightarrow X$ be a dominant rational map defined over $K$. Then the wandering points in $X_{f}(K)$ are Zariski dense in $X$.

Amerik's proof uses deep results of Hruskovski involving model theory and logic. It would be interesting to find a more elementary, dynamically inspired, proof.

Question 7.10. Is Amerik's theorem (Theorem 7.9) true for $X=\mathbb{P}^{N}$ if $K$ is a number field? And for a general variety $X$, if $K$ is a number field, is there a Zariski dense set of wandering points $P \in X_{f}(\bar{K})$ satisfying $[K(P): K] \leq C(X)$ ?

\section{8. (PRE)PERIODIC POINTS ON SUBVARIETIES}

As we have seen in Sections 4and 6, and will further explore in Sections 9 and 12 , the periodic and preperiodic points of a map form a collection of specially marked points for the map, just as the torsion points on an abelian variety are special. The classical Manin-Mumford conjecture, which was proven Raynaud [193, 194, describes the distribution of torsion points of an abelian variety $A$ lying on a subvariety of $A 14$ Replacing torsion points with preperiodic points (cf. Example 2.2) leads to various dynamical analogues.

Zhang [246, 247] formulated a version of the Manin-Mumford conjecture for polarizable endomorphisms 15 which are endomorphisms $f: X \rightarrow X$ of a projective variety that extend to noninvertible endomorphisms of the projective space in which $X$ sits; see 66]. Unfortunately, this natural conjecture turns out to be false, but we give the statement anyway because it illustrates some of the subtleties and

\footnotetext{
${ }^{13}$ The usual statement of the classical Mordell-Lang conjecture says that for any finitely generated subgroup $\Gamma \subset A(\mathbb{C})$, the Zariski closure of $Y \cap \Gamma$ in $A$ is a finite union of translates of abelian subvarieties of $A$ by points of finite order.

${ }^{14}$ More precisely, the classical Manin-Mumford conjecture that was proven by Raynaud says that if $A / \mathbb{C}$ is an abelian variety and $Y \subset A$ is a subvariety, then the Zariski closure of $Y \cap A_{\text {tors }}$ is a finite union of translates of abelian subvarieties of $A$ by points of finite order.

${ }^{15}$ Formally, $f: X \rightarrow X$ is polarizable if there is an ample line bundle $\mathcal{L}$ on $X$ and an integer $d \geq 2$ such that $f^{*} \mathcal{L} \cong \mathcal{L}^{\otimes d}$.
} 
pitfalls that can arise when generalizing conjectures and theorems from arithmetic geometry to the dynamical setting.

Conjecture 8.1 (False Dynamical Manin-Mumford Conjecture). Let $X / \mathbb{C}$ be a projective variety, let $f: X \rightarrow X$ be a polarizable morphism, and let $Y$ be a subvariety of $X$. If

$$
\#(Y \cap \operatorname{PrePer}(f))=\infty,
$$

then $Y$ contains a preperiodic subvariety of $X$ of dimension at least 1 . (See Definition 7.2 for the definition of preperiodic subvariety.)

Conjecture 8.1, although plausible, is false. One can construct a counterexample by starting with an elliptic curve $E$ having complex multiplication and taking $Y$ to be the diagonal in $E \times E$; see [94]. However, the following modification may be true.

Conjecture 8.2 (Ghioca-Tucker-Zhang, 2011, [94, Conjecture 2.4]). Let $X / \mathbb{C}$ be a projective variety, let $f: X \rightarrow X$ be a polarizable endomorphism, and let $Y$ be a subvariety of $X$ which has no component contained entirely within the singular locus of $X$. Then the following are equivalent:

(a) $Y$ is preperiodic under $f$.

(b) The intersection $Y \cap \operatorname{PrePer}_{f}(X)$ contains a Zariski dense subset of smooth points such that for every $x$ in the subset, the tangent subspace of $Y$ at $x$ is preperiodic under the induced action of $f$ on the Grassmanian bundle $\operatorname{Gr}_{\operatorname{dim}(Y)}\left(T_{X}\right)$, where we write $T_{X}$ for the tangent bundle of $X$.

Conjecture 8.2 has been proven in a few special cases, including the following.

Theorem 8.3. Conjecture 8.2 is true in the following cases.

(a) (Ghioca-Tucker-Zhang, 2011, 94]) $f$ is a group endomorphism of an abelian variety.

(b) (Ghioca-Nguyen-Ye, 2015, 91]; Ghioca-Nguyen-Ye, 2017, 92]) $f$ is an endomorphism of $\left(\mathbb{P}^{1}\right)^{n}$.

We remark that the original Conjecture 8.1 is false for both of the cases covered by Theorem 8.3 .

\section{Dynamical (Dynatomic) Modular Curves}

A natural way to study periodic points of rational maps on $\mathbb{P}^{1}$ is to classify pairs $(f, P)$, where $f$ runs over all rational maps of some fixed degree and $P$ runs over all points of some fixed period for $f$. A degree $d$ rational map $f: \mathbb{P}^{1} \rightarrow \mathbb{P}^{1}$ is described by a pair of homogeneous polynomials $f(X, Y)=[F(X, Y), G(X, Y)]$. We view the coefficients of $F$ and $G$ as indeterminates, say

$F(X, Y)=a_{0} X^{d}+a_{1} X^{d-1} Y+\cdots+a_{d} \quad$ and $\quad G(X, Y)=b_{0} X^{d}+b_{1} X^{d-1} Y+\cdots+b_{d}$, and we write the $n$th iterate of $f$ as

$$
f^{n}(X, Y)=\left[F_{n}(X, Y), G_{n}(X, Y)\right],
$$

so $F_{n}$ and $G_{n}$ are in the polynomial ring $\mathbb{Z}\left[a_{0}, \ldots, a_{d}, b_{0}, \ldots, b_{d}, X, Y\right]$. The points whose period divides $n$ are then the roots of the polynomial

$$
Y F_{n}(X, Y)-X G_{n}(X, Y)=0,
$$


but if we want to get the points of exact period $n$, we need to remove points of lower period. This is done by inclusion-exclusion, modeled after the construction of the classical cyclotomic polynomials.

Definition 9.1. With notation as above, the nth dynatomic polynomial is given by 16

$$
\Phi_{n}(X, Y):=\prod_{d \mid n}\left(Y F_{n}(X, Y)-X G_{n}(X, Y)\right)^{\mu(n / d)},
$$

where $\mu$ is the Möbius function; see Section 6. If we want to specify the dependence on $f$, we will write $\Phi_{n}(f ; X, Y)$. More generally, for any $m \geq 0$ and $n \geq 1$, we characterize preperiodic points with tail length $m$ and cycle length $n$ using the generalized $(m, n)$ dynatomic polynomial

$$
\Phi_{m, n}(X, Y):=\frac{\Phi_{n}\left(F_{m}(X, Y), G_{m}(X, Y)\right)}{\Phi_{n}\left(F_{m-1}(X, Y), G_{m-1}(X, Y)\right)} .
$$

(For $m=0$, we set $\Phi_{0, n}:=\Phi_{n}$.)

Proposition 9.2 ([214, Theorem 4.5]). The dynatomic polynomial is a polynomial. More precisely,

$$
\Phi_{n}(X, Y) \in \mathbb{Z}\left[a_{0}, \ldots, a_{n}, b_{0}, \ldots, b_{n}, X, Y\right] .
$$

For a given map $f \in \operatorname{End}_{d}^{1}(\bar{K})$, the roots of its associated dynatomic polynomial $\Phi_{n}(f ; X, Y)$ include all of the points of exact period $n$ for $f$, but it may happen that there are roots giving points of period strictly smaller than $n$. When this happens, $\Phi_{n}$ has a multiple root. Following Milnor, the roots of $\Phi_{n}(f ; X, Y)$ are said to be points of formal period $n$ for $f$.

Remark 9.3. See 116, 117 for a generalization of Proposition 9.2 to dynatomic 0 -cycles on $\mathbb{P}^{N}$ and on other varieties.

We now fix a degree $d \geq 2$ and restrict our attention to the special, but enlightening, case of polynomials maps of the form 17

$$
f_{c}(x)=x^{d}+c .
$$

The $n$th dynatomic polynomial for $f_{c}(x)$ is a polynomial in the variables $x$ and $c$. To avoid confusion, we denote it by $\Psi_{n}(x, c) \in \mathbb{Z}[x, c]$. For example, taking $d=2$ and $n=3$, we find that $\Psi_{3}(x, c)$ is given by

$$
\begin{aligned}
\Psi_{3}(x, c)=\frac{f_{c}^{3}(x)-x}{f_{c}(x)-x}= & x^{6}+x^{5}-(3 c-1) x^{4}-(2 c-1) x^{3} \\
& +\left(3 c^{2}-3 c+1\right) x^{2}+\left(c^{2}-2 c+1\right) x-c^{3}+2 c^{2}-c+1 .
\end{aligned}
$$

Before stating the next result, we need one definition.

Definition 9.4. Let $C$ be an irreducible algebraic curve. The gonality of $C$ is the smallest degree of a dominant rational map $C \rightarrow \mathbb{P}^{1}$. It is an analogue for algebraic curves of the degree of a number field over $\mathbb{Q}$. More generally, for any irreducible variety $X$ of dimension $N$, we define the gonality of $X$ to be the smallest degree of a dominant rational map $X \rightarrow \mathbb{P}^{N}$.

\footnotetext{
${ }^{16}$ Alternatively, we can set $\Phi_{1}=Y F-X G$ and define $\Phi_{n}$ recursively via the relation $\prod_{d \mid n} \Phi_{d}=$ $Y F_{n}-X G_{n}$.

${ }^{17}$ This is the dehomogenized form of the map $f_{c}(X, Y)=\left[X^{d}+c Y^{d}, Y^{d}\right]$, where we set $X=x$ and $Y=1$.
} 
Theorem 9.5. Let $d \geq 2$, let $\Psi_{n}(x, c)$ be the $n$th dynatomic polynomial associated to the map $f_{c}(x)=x^{d}+c$, and let $X_{1}^{\mathrm{dyn}}\left(f_{c} ; n\right)$ be the smooth projective curve (Riemann surface) obtained by starting with the affine curve defined by the equation $\Psi_{n}(x, z)=0$, taking its closure in $\mathbb{P}^{2}$ and then resolving the singularities. Further, let $X_{0}^{\mathrm{dyn}}\left(f_{c} ; n\right)$ be the quotient of $X_{1}^{\mathrm{dyn}}\left(f_{c} ; n\right)$ by the equivalence relation $(x, z) \sim$ $\left(f_{c}(x), z\right)$.

(a) (Bousch, 1992, 41]; Lau-Schleicher, 1994, 144, 203]; Morton, 1996, [178]) The polynomial $\Psi_{n}(x, z)$ does not factor in $\mathbb{C}[x, z]$, and hence the curves $X_{1}^{\mathrm{dyn}}\left(f_{c} ; n\right)$ and $X_{0}^{\mathrm{dyn}}\left(f_{c} ; n\right)$ are geometrically irreducible.

(b) (Morton, 1996, [178] ) There are explicit formulas for the genera of the curves $X_{1}^{\mathrm{dyn}}\left(f_{c} ; n\right)$ and $X_{0}^{\mathrm{dyn}}\left(f_{c} ; n\right)$, but they are too complicated to state here.

(c) (Doyle-Poonen, 2017, 61]) The gonalities of $X_{1}^{\mathrm{dyn}}\left(f_{c} ; n\right)$ and $X_{0}^{\mathrm{dyn}}\left(f_{c} ; n\right)$ satisfy

$$
\begin{aligned}
& \operatorname{Gonality}\left(X_{1}^{\mathrm{dyn}}\left(f_{c} ; n\right)\right) \underset{n \rightarrow \infty}{\longrightarrow} \infty, \\
& \operatorname{Gonality}\left(X_{0}^{\mathrm{dyn}}\left(f_{c} ; n\right)\right) \geq\left(\frac{1}{2}-\frac{1}{2 d}-o(1)\right) n \quad \text { as } n \rightarrow \infty .
\end{aligned}
$$

Remark 9.6. The notation $X_{1}^{\mathrm{dyn}}\left(f_{c} ; n\right)$ and $X_{0}^{\mathrm{dyn}}\left(f_{c} ; n\right)$ is modeled after the classical notation for the elliptic modular curves $X_{1}(n)$ and $X_{0}(n)$. The points of $X_{1}(n)$ classify pairs $(E, P)$, with $E$ an elliptic curve and $P \in E$ a point of exact order $n$. The subscript 1 on $X_{1}$ indicates that $X_{1}(n)$ classifies a single point of order $n$, just as $X_{1}^{\text {dyn }}\left(f_{c} ; n\right)$ classifies a single point of period $n$. More generally, there are curves $X_{1}^{\mathrm{dyn}}\left(f_{c} ; n_{1}, \ldots, n_{r}\right)$ that classify maps with marked points of several indicated periods, and even more generally, curves $X_{1}^{\text {dyn }}(\Gamma)$ that classify maps with marked points and/or cycles with orbits having a specified graph structure. See 59,62 for further details.

We next consider the set of parameter values $c$ for which the polynomial $f_{c}(x)$ is post-critically finite. The critical points of $f_{c}(x)$ are $\infty$, which is fixed, and 0 , so $f_{c}(x)$ is PCF if and only if there are integers $m \geq 0$ and $n \geq 1$ such that $\Phi_{m, n}\left(f_{c} ; 0\right)=0$. The quantity $\Phi_{m, n}\left(f_{c} ; 0\right)$ is a polynomial in $c$, and it is not hard to show that it has simple roots, but it occasionally has an extraneous factor.

Definition 9.7. Let $d \geq 2$ and $f_{d, c}(x)=x^{d}+c$. The $(m, n)$ Gleason polynomial of $f_{d, c}$ is

$$
G_{d, m, n}(c):= \begin{cases}\Phi_{m, n}\left(f_{d, c} ; 0\right) / \Phi_{0, n}\left(f_{d, c} ; 0\right)^{d-1} & \text { if } m \geq 1 \text { and } n \mid(m-1) \\ \Phi_{m, n}\left(f_{d, c} ; 0\right) & \text { otherwise. }\end{cases}
$$

The roots of $G_{d, m, n}(c)$ give the values of $c$ for which the nontrivial critical point of $x^{d}+c$ has tail length $m$ and formal period length $n$.

Question 9.8. Let $G_{d, m, n}(c) \in \mathbb{Z}[c]$ be the Gleason polynomial of $x^{d}+c$.

(a) Is it true that $G_{2, m, n}$ is irreducible in $\mathbb{Q}[c]$ for all $m \geq 0$ and $n \geq 1$ ?

(b) For which $(d, m, n)$ is $G_{d, m, n}$ irreducible in $\mathbb{Q}[c]$ ?

(c) More generally, what is the complete factorization of $G_{d, m, n}$ in $\mathbb{Q}[c]$ ?

A few partial results are known regarding Question 9.8 Goksel 97] shows that for $m \geq 1$, the Gleason polynomial $G_{d, m, n}$ is irreducible in $\mathbb{Q}[c]$ if $n=1$ and $d$ is 
prime, or if $n=d=2$. Buff [43] notes that $G_{d, 0,3}$ is reducible in $\mathbb{Q}[c]$ if and only if $d \equiv 1(\bmod 6)$. See 44 for generalizations of these results by Buff, Epstein, and Koch.

It is natural to consider dynamical modular curves associated to other interesting one-parameter families of maps, for example the maps

$$
f_{b}(x)=b x+x^{-1}
$$

discussed in Conjecture 4.7 that satisfy $f_{b}(-x)=-f_{b}(x)$. The points on the dynatomic curve associated to $f_{b}$ classify points of formal period $n$ for $f_{b}$, but in contrast to Theorem 9.5 these curves may be reducible.

Theorem 9.9 (Manes, 2009, 162]). Let $\Phi_{n}\left(f_{b} ; x\right) \in \mathbb{Z}[x, b]$ be the $n$th dynatomic polynomial associated to $f_{b}(x)=b x+x^{-1}$, viewed as a polynomial in two variables $x$ and $b$, and let $X_{1}^{\mathrm{dyn}}\left(f_{b} ; n\right)$ be the smooth projective curve obtained by completing and desingularizing the affine curve defined by the equation $\Phi_{n}\left(f_{b} ; x\right)=0$. Then for all $n \geq 2$, the curve $X_{1}^{\mathrm{dyn}}\left(f_{b} ; 2 n\right)$ has at least two irreducible components, and in fact, the polynomial $\Phi_{n}\left(f_{b} ; x\right)=0$ has at least two irreducible factors in $\mathbb{Q}[x, b]$.

Conjecture 9.10. Let $f_{b}(x)=b x+x^{-1}$ be as in Theorem 9.9.

(a) If $n \geq 1$ is odd, then the polynomial $\Phi_{n}\left(f_{b} ; x\right)=0$ is irreducible in $\mathbb{C}[x, b]$.

(b) If $n \geq 4$ is even, then the polynomial $\Phi_{n}\left(f_{b} ; x\right)=0$ has exactly two irreducible factors in $\mathbb{C}[x, b]$.

See Conjecture 10.13 for a supersized, albeit less precise, generalization of Conjecture 9.10 .

\section{Dynamical moduli SPACES}

Rather than studying iteration of a single rational function $f$, we saw in Section 9 that it is often advantageous to consider families of maps or even the family of all maps. A rational map $f: \mathbb{P}^{N} \rightarrow \mathbb{P}^{N}$ of degree $d$ is specified by an $(N+1)$-tuple

$$
f=\left[f_{0}, \ldots, f_{N}\right]
$$

of degree $d$ homogeneous polynomials $f_{0}, \ldots, f_{N} \in \bar{K}\left[X_{0}, \ldots, X_{n}\right]$, Each $f_{i}$ has the form

$$
f_{i}=\sum_{j_{0}+j_{1}+\cdots+j_{N}=d} a_{i j_{0} j_{1} \cdots j_{N}} X_{0}^{j_{0}} X_{1}^{j_{1}} \cdots X_{N}^{j_{N}}
$$

We identify $f$ with the list of coefficients $a_{i j_{0} \cdots j_{N}}$, but we note that multiplying the coefficients by a nonzero constant gives the same map $f$. Thus the map $f$ is identified with the point in projective space given by its coefficients listed in some specified order,

$$
f \longleftrightarrow\left[a_{i j_{0} j_{1} \cdots j_{N}}\right] \in \mathbb{P}^{\nu}(\bar{K}), \quad \nu=\nu(N, d):=\left(\begin{array}{c}
N+d \\
d
\end{array}\right)(N+1)-1 .
$$

Of course, not every point in $\mathbb{P}^{\nu}(\bar{K})$ gives a rational map of degree $d$, since the corresponding polynomials $f_{0}, \ldots, f_{N}$ may have a common factor, but most do, and indeed, most give a morphism, as explained by the following result. 
Theorem 10.1. The subset of $\mathbb{P}^{\nu}$ corresponding to degree $d$ morphisms of $\mathbb{P}^{N}$, which we denote by $\operatorname{End}_{d}^{N}$, is a Zariski open subset of $\mathbb{P}^{\nu} 18$

If we start with a dominant rational map $f: \mathbb{P}^{N} \rightarrow \mathbb{P}^{N}$ and make a simultaneous change-of-variables to the domain and range, we get a new map whose dynamical properties are the same as the original one. A linear change-of-variables of $\mathbb{P}^{N}$ is given by an invertible $(N+1)$-by- $(N+1)$ matrix, and two matrices determine the same variable change if they are scalar multiples of one another. Thus the automorphism group of $\mathbb{P}^{N}$ is the projective linear group

$$
\operatorname{Aut}\left(\mathbb{P}^{N}\right)=\mathrm{PGL}_{N+1} \text {. }
$$

We define an action of $\varphi \in \mathrm{PGL}_{N+1}(\bar{K})$ on a rational map $f: \mathbb{P}^{N} \rightarrow \mathbb{P}^{N}$ by setting

$$
f^{\varphi}:=\varphi^{-1} \circ f \circ \varphi .
$$

This action commutes with iteration in the sense that

$$
\left(f^{\varphi}\right)^{n}=\left(f^{n}\right)^{\varphi} \text {. }
$$

In studying families of dynamical systems on $\mathbb{P}^{N}$, we really want to study equivalence classes of maps modulo this action of $\mathrm{PGL}_{N+1}$. Identifying the set of maps $f$ with (a subset of) the projective space $\mathbb{P}^{\nu}$ as above, the conjugation action (10.1) defines a complicated, but linear, action of $\mathrm{PGL}_{N+1}$ on $\mathbb{P}^{\nu}$; more precisely, it defines a group homomorphism

$$
\mathrm{PGL}_{N+1} \longleftrightarrow \mathrm{PGL}_{\nu+1}=\operatorname{Aut}\left(\mathbb{P}^{\nu}\right) \text {. }
$$

Definition 10.2. The moduli space of degree $d$ dynamical systems on $\mathbb{P}^{N}$ is the quotient space

$$
\mathcal{M}_{d}^{N}:=\operatorname{End}_{d}^{N} / \mathrm{PGL}_{N+1} .
$$

This raises a delicate question: to what extent does the quotient $\mathcal{M}_{d}^{N}$ exist and have nice properties? This question is partially answered by the following amalgam of results.

Theorem 10.3. Let $N \geq 1$ and $d \geq 2$.

(a) (Milnor 174$) \mathcal{M}_{d}^{1}(\mathbb{C})$ exists as a complex orbifold.

(b) (Silverman 213]; Petsche-Szpiro-Tepper [189]; Levy [150]) $\mathcal{M}_{d}^{N}$ exists as a geometric quotient scheme over $\operatorname{Spec}(\mathbb{Z})$ in the sense of geometric invariant theory (GIT).

(c) (Milnor [174]; Silverman [213]) $\mathcal{M}_{2}^{1}$ is isomorphic to the affine plane $\mathbb{A}^{2}$, and its natural GIT compactification is $\mathbb{P}^{2}$.

(d) (Levy [150]) $\mathcal{M}_{d}^{1}$ is a rational variety; i.e., there exists a dominant rational map $F: \mathbb{P}^{2 d-2} \rightarrow \mathcal{M}_{d}^{1}$ such that $F$ has a dominant rational inverse.

Definition 10.4. The automorphism group of a dominant rational map $f: \mathbb{P}^{N} \rightarrow-\rightarrow$ $\mathbb{P}^{N}$ defined over $\bar{K}$ is the group

$$
\operatorname{Aut}(f):=\left\{\varphi \in \mathrm{PGL}_{N+1}(\bar{K}): f^{\varphi}=f\right\} .
$$

\footnotetext{
${ }^{18}$ More precisely, there is a single geometrically irreducible polynomial $R(f)$ in the coefficients of $f$, called the Macaulay resultant of $f$, having the property that $f \in \operatorname{End}_{d}^{N}$ if and only if $R(f) \neq 0$. For $N=1$, if we write $f=[F(X, Y), G(X, Y)]$, then $R(f)$ is just the classical resultant of $F$ and $G$.

${ }^{19} \mathrm{It}$ is also true that the subset of $\mathbb{P}^{\nu}$ corresponding to degree $d$ dominant rational maps of $\mathbb{P}^{N}$ is a Zariski open subset of $\mathbb{P}^{\nu}$; see [217, Proposition 7].
} 
It is easy to check that $\operatorname{Aut}\left(f^{\psi}\right)=\psi^{-1} \operatorname{Aut}(f) \psi$, so the $\mathrm{PGL}_{N+1}$-equivalence class of $f$ determines the group $\operatorname{Aut}(f)$ up to $\mathrm{PGL}_{N+1}$-conjugacy.

Remark 10.5. If $f$ is an endomorphism, then it is known that $\operatorname{Aut}(f)$ is finite, and indeed its order may be bounded in terms of $d$ and $N$ [150], but for dominant rational maps, the group Aut $(f)$ may be infinite.

Question 10.6. We collect some of the natural questions that one might ask about the moduli spaces $\mathcal{M}_{d}^{N}$ and about automorphism groups.

(a) For $N \geq 2$, are all (some, none) of the moduli spaces $\mathcal{M}_{d}^{N}$ rational varieties?

(b) For each $(N, d)$, describe the possible groups $\operatorname{Aut}(f)$ for $f \in \mathcal{M}_{d}^{N}$, or at least give a good upper bound for

$$
U(N, d):=\sup _{f \in \mathcal{M}_{d}^{N}} \# \operatorname{Aut}(f)
$$

in terms of $N$ and $d$. It is known that $U(N, d)$ is finite [150, that $U(1, d) \leq$ $\max \{60,2 d+2\}$ [218, Example 2.54], and that $U(2, d) \leq 60 d^{6}$ [52, Theorem 6.2].

(c) Describe the subvarieties of $\mathcal{M}_{d}^{N}$ corresponding to maps $f$ whose automorphism group Aut $(f)$ is nontrivial. See [173] for a detailed analysis for $N=1$ and 163 for some partial results for $N=d=2$. For example,

$$
\left\{f \in \mathcal{M}_{2}^{1} \cong \mathbb{A}^{2}: \operatorname{Aut}(f) \neq 1\right\}=\text { cuspidal cubic curve in } \mathbb{A}^{2} \text {, }
$$

with $\operatorname{Aut}(f) \cong \mathbb{Z} / 2 \mathbb{Z}$ except for the map $f$ at the cusp, for which $\operatorname{Aut}(f) \cong$ $\mathcal{S}_{3}$.

(d) Most of the spaces $\mathcal{M}_{d}^{N}$ are singular. Describe the singularity locus. The case $N=1$ is analyzed in 173 .

An arithmetic question closely related to dynamical moduli spaces is that of fields of definition versus field of moduli, a problem that also arises in the theory of abelian varieties.

Definition 10.7. Let $f \in \operatorname{End}_{d}^{N}(\overline{\mathbb{Q}})$, and let

$$
\pi: \operatorname{End}_{d}^{N}(\overline{\mathbb{Q}}) \longrightarrow \mathcal{M}_{d}^{N}(\overline{\mathbb{Q}})
$$

be the natural projection map. The field of moduli of $f$ is the field $\mathbb{Q}(\pi(f))$, i.e., the field generated by the coordinates of the point $\pi(f)$. A field of definition for $f$ is any field $L$ for which there is some $\varphi \in \operatorname{PGL}_{N+1}(\overline{\mathbb{Q}})$ such that $f^{\varphi} \in \operatorname{End}_{d}^{N}(L)$.

It is easy to see that every field of definition of $f$ contains its field of moduli, but there exist maps whose field of moduli is not a field of definition. For example, one can show that the field of moduli of the map $f(x)=i\left(x^{3}-1\right) /\left(x^{3}+1\right)$ is $\mathbb{Q}$, although $\mathbb{Q}$ is not a field of definition for $f$.

Definition 10.8. For $f \in \operatorname{End}_{d}^{N}(\overline{\mathbb{Q}})$, let $K_{f}$ denote the field of moduli of $f$, and define

$$
\mathrm{FOM} / \mathrm{FOD}(N, d):=\sup _{f \in \operatorname{End}_{d}^{N}(\overline{\mathbb{Q}})} \inf _{\begin{array}{l}
L \text { is a field of } \\
\text { definition of } f
\end{array}}\left[L: K_{f}\right] .
$$

In other words, $\operatorname{FOM} / \operatorname{FOD}(N, d)$ is the smallest integer $D$ such that every map in $\operatorname{End}_{d}^{N}(\overline{\mathbb{Q}})$ has a field of definition whose degree over its field of moduli is bounded by $D$. Of course, it is not clear a priori that $\operatorname{FOM} / \operatorname{FOD}(N, d)$ is finite. 


\section{Theorem 10.9.}

(a) For $N=1$ we have the exact values

$$
\mathrm{FOM} / \mathrm{FOD}(1, d)= \begin{cases}1 & \text { if } d \geq 2 \text { is even (Silverman, 1995, [211]), } \\ 2 & \text { if } d \geq 3 \text { is odd (Hidalgo, 2014, [110]). }\end{cases}
$$

(b) For all $N \geq 1$ and all $d \geq 2$, the quantity $\operatorname{FOM} / \operatorname{FOD}(N, d)$ is finite (DoyleSilverman, 2018, 63]).

The fact that the bound for $\mathrm{FOM} / \mathrm{FOD}(1, d)$ does not depend on $d$ suggests the following question.

Question 10.10. For a given $N \geq 1$, is

$$
\sup _{d \geq 2} \operatorname{FOM} / \operatorname{FOD}(N, d)<\infty ?
$$

If so, what is its value as a function of $N$ ?

Just as we did for dynamical modular curves in Section 9, we can define moduli spaces with level structure by specifying maps together with points or cycles of various shapes. For simplicity, we restrict our discussion to the case of a single periodic point.

Definition 10.11. Let $N \geq 1, n \geq 1$, and $d \geq 2$. We recall that $\operatorname{Per}_{n}^{*}(f)$ denotes the set of points of exact period $n$ for $f$, and we define

$$
\operatorname{End}_{d}^{N}[n]:=\left\{(f, P): f \in \operatorname{End}_{d}^{N} \text { and } P \in \operatorname{Per}_{n}^{*}(f)\right\} \subset \operatorname{End}_{d}^{N} \times \mathbb{P}^{N}
$$

to be the space that classifies maps with a marked point of exact period $n$. We define an action of $\varphi \in \mathrm{PGL}_{N+1}$ on $(f, P) \in \operatorname{End}_{d}^{N}[n]$ by the rule

$$
(f, P)^{\varphi}:=\left(f^{\varphi}, \varphi^{-1}(P)\right)
$$

and we let

$$
\mathcal{M}_{d}^{N}[n]:=\operatorname{End}_{d}^{n}[n] / \mathrm{PGL}_{N+1}
$$

be the associated quotient space. There is a natural map

$$
i_{n}: \mathcal{M}_{d}^{N}[n] \rightarrow \mathcal{M}_{d}^{N} \quad \text { induced by }(f, P) \mapsto f .
$$

Example 10.12. Let $N=1$ and $d=2$, so $\mathcal{M}_{2}^{1} \cong \mathbb{A}^{2}$. The set of polynomial maps $\left\{f_{c}(x):=x^{2}+c\right\}$ gives a curve $L$ in $\mathcal{M}_{2}^{1}$, and Theorem 9.5 says that for all $n \geq 1$, the inverse image curve $i_{n}^{-1}(L) \cong X_{1}^{\mathrm{dyn}}\left(f_{c} ; n\right)$ is irreducible, and that its genus and gonality go to infinity as $n \rightarrow \infty$. Similarly, the set of rational maps $\left\{f_{b}(x):=b x+x^{-1}\right\}$ gives a curve $C$ in $\mathcal{M}_{2}^{1}$, and Theorem 9.9 says that for all even $n \geq 2$, the inverse image curve $i_{n}^{-1}(C) \cong X_{1}^{\text {dyn }}\left(f_{c} ; n\right)$ has at least two irreducible components.

Dynamical modular curves associated to natural one-parameter families of maps may be reducible. The following conjecture, the first part of which was raised as a question by Poonen, says that there exists a uniform bound for the amount of reducibility as we add more level structure.

Conjecture 10.13. Let $N \geq 1$, let $d \geq 2$, and let $\Gamma \subset \mathcal{M}_{d}^{N}$ be an irreducible subvariety, e.g., $\Gamma$ could be an irreducible curve. For each $n \geq 1$, write $i_{n}^{-1}(\Gamma)$ as a union of irreducible varieties,

$$
i_{n}^{-1}(\Gamma)=\Gamma_{n, 1} \cup \Gamma_{n, 2} \cup \cdots \cup \Gamma_{n, \nu}, \quad \text { where } \nu=\nu(N, d, n ; \Gamma) .
$$


(a) (Poonen, 2012, [192]) The number of irreducible components of $i_{n}^{-1}(\Gamma)$ is bounded independently of $\Gamma$ and $n$, i.e., there is a constant $C_{1}(N, d)$ such that

$$
\sup _{\text {irreducible } \Gamma \subset \mathcal{M}_{d}^{N}} \nu(N, d, n ; \Gamma) \leq C_{1}(N, d) .
$$

(b) There is a constant $C_{2}(N, d)$ such that for all $n \geq C_{2}(N, d)$, all irreducible $\Gamma \subset \mathcal{M}_{d}^{N}$, and all $1 \leq j \leq \nu(N, d, n \Gamma)$, the variety $\Gamma_{n, j}$ is of general type 20

(c) The gonality (see Definition 9.4) of the components of $i_{n}^{-1}(\Gamma)$ satisfy

$$
\lim _{n \rightarrow \infty} \min _{1 \leq i \leq \nu(N, d, n ; \Gamma)} \operatorname{Gonality}\left(\Gamma_{n, i}\right)=\infty .
$$

(d) Let $\Gamma$ be an irreducible curve, so $i_{n}^{-1}(\Gamma)$ is a union of irreducible curves. Then

$$
\lim _{n \rightarrow \infty} \min _{1 \leq i \leq \nu(N, d, n ; \Gamma)} \operatorname{Genus}\left(\Gamma_{n, i}\right)=\infty .
$$

Question 10.14. In Conjecture 10.13(c), (d) give (accurate) lower bounds for the genera and gonalities of the components $\Gamma_{n, i}$ as $n \rightarrow \infty$ when $\Gamma$ is an irreducible curve.

\section{UNLIKELY INTERSECTIONS IN DYNAMICS}

In Sections 7 and 8 we discussed subvarieties containing a large number of preperiodic points or a large number of points in an orbit. An orbit $\mathcal{O}_{f}(P)$ consists of a countable number of points, as does the set $\operatorname{PrePer}(f)$ of preperiodic points, and the theme in those sections was that it is unlikely for these countable sets to have large intersection with a proper subvariety unless there is a geometric reason to expect the intersection to be large.

The study of unlikely intersections in arithmetic geometry has seen a surge of interest, and there are many analogous problems that can be formulated for dynamical systems. The guiding principle is that families of objects should intersect infrequently unless there is a geometric reason forcing the converse.

The following theorem, which is a dynamical analogue of a theorem of Masser and Zannier for elliptic curves [165, illustrates the type of result that one seeks. It it worth noting that the finiteness in Theorem 11.1 takes place in moduli space, i.e., in the $t$-space that parameterizes the quadratic polynomials $x^{2}+t$.

Theorem 11.1 (Baker-DeMarco, 2011, 9]). For $t \in \mathbb{C}$, let $f_{t}(x)=x^{2}+t$ be the usual quadratic polynomial. Let $a, b \in \mathbb{C}$ be complex numbers with $a^{2} \neq b^{2}$. Then

$$
\left\{t \in \mathbb{C}: a \in \operatorname{PrePer}\left(f_{t}\right) \text { and } b \in \operatorname{PrePer}\left(f_{t}\right)\right\} \text { is finite. }
$$

More generally, we might allow the points and the maps to vary with the parameter. So let $T$ be a nonsingular algebraic curve, and let

$$
a: T \longrightarrow \mathbb{P}^{1}, \quad b: T \longrightarrow \mathbb{P}^{1}, \quad f: T \longrightarrow \operatorname{End}_{d}^{1}
$$

be nonconstant algebraic maps. We view $a_{t}$ and $b_{t}$ as defining families of points parameterized by $t \in T$, and similarly we view $f$ as a family of rational maps $f_{t}: \mathbb{P}^{1} \rightarrow \mathbb{P}^{1}$ parameterized by $t \in T$.

\footnotetext{
${ }^{20}$ The precise definition of general type is somewhat technical, although for a smooth curve it simply means that the genus is at least 2 . In general, suffice it to say that general type implies that the geometry of $\Gamma_{n, j}$ is very complicated.
} 
The independence of $a$ and $b$ in Theorem 11.1 which we stated as $a^{2} \neq b^{2}$, is equivalent to saying that $f_{t}(a) \neq f_{t}(b)$. More generally, for varying families of maps and points, we want to define what it means for $a$ and $b$ to be $f$-dynamically related. For example, this will include the case that $f^{n} \circ a=f^{m} \circ b$ for some $n \geq 0$ and $m \geq 0$, but this is not the only situation. For example, if there is some $\varphi: T \rightarrow$ End $_{d}^{1}$ that commutes with $f$, i.e., $f_{t} \circ \varphi_{t}=\varphi_{t} \circ f_{t}$ for all $t \in T$, then $a$ and $\varphi \circ a$ are dynamically related for every $a$. Similarly, if $a_{t} \in \operatorname{PrePer}\left(f_{t}\right)$ for every $t \in T$, then $a$ is dynamically related to every $b$. The varied nature of these examples gives some justification for the technical nature of the following formal definition.

Definition 11.2. Let $T$ be a nonsingular algebraic curve, and let $a, b$, and $f$ be maps as in (11.1). Let $\overline{k(T)}$ be an algebraic closure of the function field of $T$ so we may view $a$ and $b$ as elements of $\mathbb{P}^{1}(\overline{k(T)})$, and we may view $f$ as a map $f: \mathbb{P}^{1}(\overline{k(T)}) \rightarrow \mathbb{P}^{1}(\overline{k(T)})$. Then we say that $a$ and $b$ are $f$-dynamically related if there is an algebraic curve

$$
C \subset\left(\mathbb{P}^{1} \times \mathbb{P}^{1}\right)(\overline{k(T)})
$$

satisfying

$$
(a, b) \in C \quad \text { and } \quad(f \times f)(C) \subseteq C .
$$

Conjecture 11.3 (Ghioca-Hsia-Tucker, 2013, 89]; DeMarco, 2016, [53]). Let T be an irreducible nonsingular algebraic curve, and let $a, b$, and $f$ be maps as in (11.1) for some $d \geq 2$ and with $k$ an algebraically closed field. Then the following are equivalent.

(a) The set $\left\{t \in T(k): a_{t}\right.$ and $b_{t}$ are both in $\left.\operatorname{PrePer}\left(f_{t}\right)\right\}$ is infinite.

(b) The maps $a$ and $b$ are $f$-dynamically related.

Using the theory of canonical heights, which is defined and discussed in Section 15, one can formulate a strengthened version of Conjecture 11.3. see Conjecture 15.4

We recall that $P \in \mathbb{P}^{1}$ is a critical point for a rational map $f: \mathbb{P}^{1} \rightarrow \mathbb{P}^{1}$ if $f$ fails to be locally one-to-one in a neighborhood of $P$, and that $f$ is said to be PCF if all of its critical points are preperiodic. PCF maps appeared briefly in Section 5 and will reappear in Section [15. As suggested by Table 1 in the Introduction, we view the collection of PCF maps in the moduli space $\mathcal{M}_{d}^{1}$ as being in some ways analogous to the collection of complex multiplication (CM) abelian varieties in the moduli space $\mathcal{A}_{g}$ of abelian varieties. In the setting of abelian varieties, there are famous conjectures of André and Oort which assert that a subvariety $X \subset \mathcal{A}_{g}$ contains a Zariski dense set of CM points if and only if there is an underlying geometric reason. In evocative terminology, the classical André-Oort conjecture is often phrased as saying that special points of $\mathcal{A}_{g}$ are Zariski dense in $X$ if and only if $X$ itself is special. This formulation would have an obvious dynamical analogue if only we knew exactly what it means for $X \subset \mathcal{M}_{d}^{1}$ to be a dynamically special subvariety!

In order to formulate a more precise conjecture, we return to the construction of the spaces $\operatorname{End}_{d}^{1}$ and $\mathcal{M}_{d}^{1}$ in Section 10. A map $f \in \operatorname{End}_{d}^{1}$ has exactly $2 d-2$ critical points, counted with multiplicities. We define

$$
\operatorname{End}_{d}^{\text {crit }}:=\left\{\left(f, P_{1}, \ldots, P_{2 d-2}\right): \begin{array}{l}
f \in \operatorname{End}_{d}^{1}, \text { and } P_{1}, \ldots, P_{2 d-2} \\
\text { are critical points of } f
\end{array}\right\}
$$


to be the space of maps with marked critical points. The group $\mathrm{PGL}_{2}$ acts as usual; cf. the construction of $\mathcal{M}_{d}^{N}[n]$ in Section [10, and one can construct the GIT quotient space $\mathcal{M}_{d}^{\text {crit }}:=\operatorname{End}_{d}^{\text {crit }} / \mathrm{PGL}_{2} 21$

Conjecture 11.4 (Dynamical André-Oort Conjecture). (Baker-DeMarco, $\approx 2011$, [8, 218]) Let $X \subseteq \mathcal{M}_{d}^{\text {crit }}$ be an algebraic subvariety such that the PCF maps in $X$ are Zariski dense in $X$. Then $X$ is cut out by critical orbit relations.

Formulas of the form $f^{n}\left(P_{i}\right)=f^{m}\left(P_{j}\right)$ define critical point relations, but other relations may arise from symmetries of $f$, and even subtler relations can come from hidden relations due to subdynamical systems. See [218, Remark 6.58] for an example due to Ingram. Although there is not, as yet, a complete description of what is meant by the phrase "critical orbit relations" in Conjecture 11.4, we note that Conjecture 11.3 implies the following precise conjecture for one-dimensional families.

Conjecture 11.5 (Dynamical André-Oort Conjecture for One-dimensional Families: Baker-DeMarco, 2013, 8]). Let T be a nonsingular algebraic curve, and let

$$
T \longrightarrow \mathcal{M}_{d}^{\text {crit }}, \quad t \longmapsto\left(f_{t}, P_{1, t}, \ldots, P_{2 d-2, t}\right)
$$

be a family of rational maps with marked critical points. Then the following are equivalent.

(a) There are infinitely many $t \in T$ such that $f_{t}$ is $P C F$.

(b) For every $i$ and $j$, the maps $P_{i}: T \rightarrow \mathbb{P}^{1}$ and $P_{j}: T \rightarrow \mathbb{P}^{1}$ are $f$-dynamically related.

Building on a number of earlier partial results $8,9,70,89,96$, Favre and Gauthier have recently announced a proof of Conjecture 11.5 for all families of polynomials, but the conjecture remains open for families of rational maps.

The next result, although it is not a special case of Conjecture 11.5 has a similar André-Oort flavor.

Theorem 11.6 (Ghioca-Krieger-Nguyen-Ye, 2017, 90]). Fix $d \geq 2$. Let $C \subset \mathbb{A}^{2}$ be an irreducible curve, and suppose that there are infinitely $(a, b) \in C(\mathbb{C})$ such that both of the maps $x^{d}+a$ and $x^{d}+b$ are PCF. Then one of following is true.

(1) $C=\left\{a=a_{0}\right\}$ and $x^{d}+a_{0}$ is PCF.

(2) $C=\left\{b=b_{0}\right\}$ and $x^{d}+b_{0}$ is PCF.

(3) $C=\{a=\zeta b\}$ for some $\zeta$ satisfying $\zeta^{d-1}=1$.

\section{GOOD REDUCTION OF MAPS AND ORBITS}

Let $p \in \mathbb{Z}$ be a prime. In this section we use tildes to denote the reduction modulo $p$ map,

$$
\mathbb{Z} \longrightarrow \mathbb{F}_{p}, \quad c \longmapsto \tilde{c}
$$

and if we need to specify the prime $p$, we write $\tilde{c} \bmod p$. Similarly, we define a reduction modulo $p$ map

$$
\mathbb{Z}\left[X_{0}, \ldots, X_{N}\right] \longrightarrow \mathbb{F}_{p}\left[X_{0}, \ldots, X_{N}\right], \quad F\left(X_{0}, \ldots, X_{N}\right) \longmapsto \tilde{F}\left(X_{0}, \ldots, X_{N}\right),
$$

obtained by reducing the coefficients of a polynomial.

\footnotetext{
${ }^{21} \mathrm{We}$ are being slightly imprecise here. It is easy to construct the GIT quotient moduli space of maps $f$ having $2 d-2$ distinct marked critical points, but one must be more careful when dealing with critical points that have higher multiplicity. See 62, for example, for a discussion.
} 
Definition 12.1. Let $P \in \mathbb{P}^{N}(\mathbb{Q})$. The reduction of $P$ modulo $p$, denoted $\tilde{P}$, is defined as follows. First choose homogeneous coordinates $P=\left[c_{0}, \ldots, c_{N}\right]$ for $P$ satisfying

and then set

$$
c_{0}, \ldots, c_{N} \in \mathbb{Z} \text { and } \operatorname{gcd}\left(c_{0}, \ldots, c_{N}\right)=1 \text {, }
$$

$$
\tilde{P}=\left[\tilde{c}_{0}, \ldots, \tilde{c}_{N}\right] \in \mathbb{P}^{N}\left(\mathbb{F}_{p}\right) .
$$

This gives a well-defined reduction modulo $p \operatorname{map} \mathbb{P}^{N}(\mathbb{Q}) \rightarrow \mathbb{P}^{N}\left(\mathbb{F}_{p}\right)$.

Definition 12.2. Let $f \in \operatorname{End}_{d}^{N}(\mathbb{Q})$, i.e., $f: \mathbb{P}^{N} \rightarrow \mathbb{P}^{N}$ is a morphism defined over $\mathbb{Q}$. The reduction of $f$ modulo $p$, denoted $\tilde{f}$, is defined as follows. First write $f$ in the form

$$
f=\left[f_{0}, \ldots, f_{N}\right] \quad \text { with } f_{0}, \ldots, f_{N} \in \mathbb{Z}\left[X_{0}, \ldots, X_{N}\right],
$$

and if necessary, divide $f_{0}, \ldots, f_{N}$ by an appropriate integer to ensure that the gcd of the collection of all of the coefficients of $f_{0}, \ldots, f_{N}$ is equal to 1 . Such an $f$ is said to be written in normalized form. We then set

$$
\tilde{f}=\left[\tilde{f}_{0}, \ldots, \tilde{f}_{N}\right] \quad \text { with } \quad \tilde{f}_{0}, \ldots, \tilde{f}_{N} \in \mathbb{F}_{p}\left[X_{0}, \ldots, X_{N}\right]
$$

where $\tilde{f}_{i}$ is obtained by reducing the coefficients of $f_{i}$ modulo $p$.

Remark 12.3. The fact that a map $f=\left[f_{0}, \ldots, f_{N}\right] \in \operatorname{End}_{d}^{N}(\mathbb{Q})$ has degree $d$ means that $f_{0}, \ldots, f_{N}$ are homogeneous polynomials of degree $d$ having no common factors in $\mathbb{Q}\left[X_{0}, \ldots, X_{N}\right]$. However, when we reduce modulo $p$, it may happen that the polynomials pick up a common factor. For example, $F=X^{2}+3 Y^{2}$ and $G=X^{2}+X Y$ have no common factors in $\mathbb{Q}[X, Y]$, but reducing modulo 3 , we find that $\tilde{F} \bmod 3=X^{2}$ and $\tilde{G} \bmod 3=X^{2}+X Y$ acquire a common factor of $X$ in $\mathbb{F}_{3}[X, Y]$. Thus $\tilde{f}(X, Y)=\left[X^{2}, X^{2}+X Y\right]=[X, X+Y]$ has degree 1 , which shows that reduction modulo $p$ does not necssarily respect the degree of a map.

Definition 12.4. Let $f: \mathbb{P}^{N} \rightarrow \mathbb{P}^{N}$ be a morphism defined over $\mathbb{Q}$, and let $p$ be a prime. The map $f$ has (naive 2 good reduction modulo $p$ if

$$
\operatorname{deg}(\tilde{f} \bmod p)=\operatorname{deg}(f) .
$$

The following elementary result illustrates the utility of good reduction. In words, it says that if $f$ has good reduction, then reduction commutes with both iteration and evaluation.

Proposition 12.5. Let $f: \mathbb{P}^{N} \rightarrow \mathbb{P}^{N}$ be a rational map defined over $\mathbb{Q}$, let $n \geq 1$, let $P \in \mathbb{P}^{N}(\mathbb{Q})$, and let $p$ be a prime of good reduction for $f$. Then $f^{n}$ has good reduction, and we have

$$
\widetilde{f^{n}}=\tilde{f}^{n} \quad \text { and } \widetilde{f^{n}(P)}=\tilde{f}^{n}(\tilde{P}) .
$$

Let $f$ have good reduction, and let $P \in \mathbb{P}^{N}(\mathbb{Q})$ be an $f$-periodic point of pe$\operatorname{riod} n$. It follows easily from Proposition 12.5 that $\tilde{P}$ is $\tilde{f}$-periodic and that its $\tilde{f}$-period divides $n$. It is possible to say much more. The following theorem is an amalgamation of results. We have stated it for maps of $\mathbb{P}^{N}$ defined over $\mathbb{Q}$, but it holds much more generally for self-maps of algebraic varieties over number fields; see especially [115.

\footnotetext{
${ }^{22}$ We discuss later why this definition is naive.
} 
Theorem 12.6 (Morton-Silverman, 1994/5, 179, 180; Pezda, 1994, 190; Zieve, 1996, 248; Hutz, 2009, [115). Let $f: \mathbb{P}^{N} \rightarrow \mathbb{P}^{N}$ be a morphism defined over $\mathbb{Q}$, let $p$ be a prime of good reduction for $f$, let $P \in \mathbb{P}^{N}(\mathbb{Q})$ be an $f$-periodic point of exact period $n$, and let $m$ be the exact period of the reduced point $\tilde{P} \in \mathbb{P}^{N}\left(\mathbb{F}_{p}\right)$ for the reduced map $\tilde{f}$. Then

$$
n=m \quad \text { or } \quad n=m r \quad \text { or } \quad n=m r p^{e},
$$

where $r$ divides $p^{N}-1$, and where there is an explicit upper bound for $e$. (For example, if $N=1$ and $p \geq 3$, then $e \leq 1$.)

Using the fact that $m$ in Theorem 12.6 is at most $\# \mathbb{P}^{N}\left(\mathbb{F}_{p}\right)$, we can use Theorem 12.6 to prove a semi-uniform version of the Dynamical Uniform Boundedness Conjecture (Conjecture 4.2) for periodic points. More precisely, the theorem gives a bound for \# $\operatorname{Per}\left(f, \mathbb{P}^{N}(K)\right)$ that depends only on $N, \operatorname{deg}(f),[K: \mathbb{Q}]$, and the number of primes at which $f$ has bad reduction. So in lieu of full uniform boundness, one might ask the following question.

Question 12.7. Find a nice function $C(N, d, D, s)$ so that every degree $d$ morphism $f: \mathbb{P}^{N} \rightarrow \mathbb{P}^{N}$ defined over a field $K$ of degree at most $D$ and having at most $s$ primes of bad reduction satisfies

$$
\text { \# } \operatorname{PrePer}\left(f, \mathbb{P}^{N}(K)\right) \leq C(N, d, D, s) .
$$

If one restricts to polynomial maps on $\mathbb{P}^{1}$, then Benedetto 31 proves that one can take $C_{\text {poly }}(1, d, D, s)$ equal to a mutiple of $d^{3}(D+s) \log (D+s)$, while Canci and Paladino [4] give a weaker bound for rational maps on $\mathbb{P}^{1}$.

We called our earlier definition of good reduction the naive definition. The reason it is naive is due to the fact that conjugating a map $f: \mathbb{P}^{N} \rightarrow \mathbb{P}^{N}$ by a linear transformation $\varphi \in \mathrm{PGL}_{N+1}$ gives a map $f^{\varphi}$ having the same dynamical behavior as $f$. But when we are reducing modulo $p$, conjugation by an element of $\mathrm{PGL}_{N+1}(\mathbb{Q})$ may turn good reduction into bad reduction, or vice versa.

Example 12.8. The map

$$
f(X, Y)=\left[p X^{2}+X Y, Y^{2}\right]
$$

has bad reduction at $p$, since $\tilde{f}=\left[X Y, Y^{2}\right]=[X, Y]$. But if we conjugate $f$ by the linear map $\varphi(X, Y)=[X, p Y]$, we find that

$$
\begin{array}{r}
f^{\varphi}(X, Y)=\varphi^{-1} \circ f \circ \varphi(X, Y)=\varphi^{-1} \circ f(X, p Y)=\varphi^{-1}\left(p X^{2}+p X Y, p^{2} Y^{2}\right) \\
=\left[p X^{2}+p X Y, p Y^{2}\right]=\left[X^{2}+X Y, Y^{2}\right] .
\end{array}
$$

Thus $f^{\varphi}$ has (naive) good reduction, so it would have been advisable to use $f^{\varphi}$ instead of $f$. This leads to a better definition.

Definition 12.9. Let $f: \mathbb{P}^{N} \rightarrow \mathbb{P}^{N}$ be a rational map defined over $\mathbb{Q}$, and let $p$ be a prime. We say that $f$ has good reduction modulo $p$ if there is a change of variables $\varphi \in \mathrm{PGL}_{N+1}(\mathbb{Q})$ such that the conjugate map $f^{\varphi}$ has good reduction modulo $p$.

Remark 12.10. There are algorithms due to Benedetto [32, Bruin-Molnar 42], Rumely [198, and Szpiro-Tepper-Williams 2232, using a variety of methods, that find a change of variables for a given map $f$ on $\mathbb{P}^{1}$ making the bad reduction as good as possible, and in particular determining if $f$ has good reduction. They play the role in arithmetic dynamics of a similar algorithm of Tate 234 that finds minimally bad equations for elliptic curves. 
A famous theorem of Shafarevich [216, Theorem IX.6.1] says that if $S$ is a finite set of primes in the ring of integers of a number field $K$, then there are only finitely many elliptic curves defined over $K$ having good reduction at all primes not in $S$. Shafarevich further conjectured, and Faltings 67. proved, the much deeper result that the same is true for abelian varieties of arbitrary fixed dimension. For those who are familiar with these theorems, it may come as a surprise that there are lots of maps $f: \mathbb{P}^{N} \rightarrow \mathbb{P}^{N}$ that have good reduction at every prime. For example, if $f: \mathbb{P}^{1} \rightarrow \mathbb{P}^{1}$ has the dehomogenized form

$$
f(x)=x^{d}+a_{1} x^{d-1}+\cdots+a_{d} \text { with } a_{1}, \ldots, a_{d} \in \mathbb{Z},
$$

then $f$ has good reduction at every prime, since it is clear that $\operatorname{deg}(\tilde{f} \bmod p)=d$ for all $p$. Thus a naive generalization of Shafarevich's conjecture to dynamical systems is false.

However, polynomial maps (12.1) form only a small part of the totality of rational maps on $\mathbb{P}^{1}$. Thus one might ask, within the collection of all dynamical systems, how widespread are the maps having good reduction outside some fixed finite set of places. As in Section 10, we parameterize degree $d$ dynamical systems on $\mathbb{P}^{N}$ by the points of the variety $\mathcal{M}_{d}^{N}$. Then for example, we have $\operatorname{dim} \mathcal{M}_{d}^{1}=2 d-2$, while the set of good reduction polynomial maps (12.1) in $\mathcal{M}_{d}^{1}$ lies on a subvariety of dimension $d-1$.

Definition 12.11. Let $N \geq 1$ and $d \geq 2$. The Shafarevich dimension for degree $d$ dynamical systems on $\mathbb{P}^{N}$, denoted $\operatorname{ShafDim}_{d}^{N}$, is the quantity

$$
\sup _{\substack{K \text { a number field } \\ S \text { a finite set of primes }}} \operatorname{dim} \overline{\left\{f \in \mathcal{M}_{d}^{N}(K): f \text { has good reduction at all primes not in } S\right\}},
$$

where the overline denotes the Zariski closure.

Good reduction polynomials (12.1) show that $\operatorname{ShafDim}_{d}^{1} \geq d-1$. The next result gives a small improvement, while suggesting a fundamental question.

Proposition 12.12. (Silverman, 2017, 208, Proposition 12]) The Shafarevich dimension for degree d dynamical systems on $\mathbb{P}^{1}$ satisfies

$$
\operatorname{ShafDim}_{2}^{1}=2, \quad \operatorname{ShafDim}_{3}^{1}=4, \quad \text { and } \quad \operatorname{ShafDim}_{d}^{1} \geq d+1 \quad \text { for all } d \geq 4 .
$$

Question 12.13. What is the exact value of $\operatorname{ShafDim}_{d}^{N}$ ? In particular, is it true that

$$
\operatorname{ShafDim}_{d}^{1}=d+1 \text { for all } d \geq 3 \text { ? }
$$

Remark 12.14. Petsche [187] shows that for certain codimension 3 families in $\mathcal{M}_{d}^{1}$, there are only finitely many maps having good reduction outside of $S$. We also mention that Petsche and Stout [188, more or less define the Shafarevich dimension ShafDim ${ }_{d}^{1}$ on $\mathbb{P}^{1}$ and ask if ShafDim ${ }_{d}^{1}=2 d-2$, i.e., they ask if good reduction maps are Zariski dense in $\mathcal{M}_{d}^{1}$. They prove that this is true for $d=2$.

Remark 12.15. Two maps $f_{1}, f_{2} \in \operatorname{End}_{d}^{N}(K)$ are said to be $K$-twists of one another if there is a $\bar{K}$-change of variables $\varphi \in \mathrm{PGL}_{N+1}(\bar{K})$ such that $f_{2}=f_{1}^{\varphi}$. We consider $f_{1}$ and $f_{2}$ to be $K$-equivalent if it is possible to find such a $\varphi$ in $\operatorname{PGL}_{N+1}(K)$. For example, the maps $f_{1}(x)=x^{3}$ and $f_{2}(x)=2 x^{3}$ are inequivalent $\mathbb{Q}$-twists of one another, since $f_{2}(x)=f_{1}^{\varphi}(x)$ for $\varphi(x)=\sqrt{2} x$. Stout [229] has proven that for a given $f \in \operatorname{End}_{d}^{N}(K)$, there are only finitely many equivalence classes of maps in $\operatorname{End}_{d}^{N}(K)$ that are $K$-twists of $f$ and have good reduction at all primes not in $S$. 
For any finite set of primes $S$, we have seen that there are infinitely many selfmaps $f$ of $\mathbb{P}^{N}$ having good reduction at all primes not in $S$. As noted earlier, this means that the natural dynamical analogue of the Shafarevich-Faltings finiteness theorem for abelian varieties is false. Various authors have attempted to rescue the finiteness by adding a condition that various parts of certain $f$-orbits have good reduction. In order to state a sharp result for maps of $\mathbb{P}^{1}$, we need a definition, which we state for general number fields.

Definition 12.16. Let $K$ be a number field, let $S$ be a finite set of primes of the ring of integers of $K$, and let $R_{S}$ be the ring of $S$-integers of $K$. For $n \geq 1$ and $d \geq 1$, define $\mathcal{G R}_{d}^{1}[n](K, S)$ to be the set of triples $(f, Y, X)$, where $f \in \operatorname{End}_{d}^{1}(K)$ and $Y \subseteq X \subset \mathbb{P}^{1}(K)$ are finite sets, satisfying the following conditions.

- $X=Y \cup f(Y)$.

- $X$ is $\operatorname{Gal}(\bar{K} / K)$-invariant.

- $\sum_{P \in Y} e_{f}(P)=n$, where $e_{f}(P)$ is the ramification index of $f$ at $P$.

- $f$ has good reduction at all primes not in $S$.

- The points in $X$ remain distinct when reduced modulo $\mathfrak{p}$ for every prime $\mathfrak{p} \notin S$.

There is a natural action of $\mathrm{PGL}_{2}\left(R_{S}\right)$ on $\mathcal{G R}_{d}^{1}[n](K, S)$, where $\mathrm{PGL}_{2}\left(R_{S}\right)$ is (essentially) the group of 2-by-2 matrices $\varphi$ with $\operatorname{det}(\varphi) \in R_{S}^{*}$. This action is given by the formula

$$
\varphi \cdot(f, Y, X)=\left(f^{\varphi}, \varphi^{-1}(Y), \varphi^{-1}(X)\right) .
$$

The following gives a strong Dynamical Shafarevich Theorem for $\mathbb{P}^{1}$ with orbit points (see also earlier work of Szpiro-Tucker, 2008, 2233]; Szpiro-West, 2017, 2231]; and Petsche-Stout, 2015, 188]).

Theorem 12.17 (Silverman, 2017, 208]). Let $K / \mathbb{Q}$ be a number field, let $S$ be a finite set of primes of $K$, and let $d \geq 2$. Then for all $n \geq 2 d+1$, the set

$$
\mathcal{G R}_{d}^{1}[n](K, S) / \mathrm{PGL}_{2}\left(R_{S}\right) \text { is finite. }
$$

Question 12.18. Is there a natural generalization of Theorem 12.17 to maps of $\mathbb{P}^{N}$ ? (We mention that the naive generalization is false; see [208, Section 8].)

It is not hard to construct examples showing that Theorem 12.17 is false for $n=$ $2 d$; see [208, Proposition 11]. However, if one further specifies the exact orbit structure of the map $f: Y \rightarrow X$, then some configurations with $n=2 d$ permit infinitely many good reduction triples, while other configurations allow only finitely many. Without giving precise definitions, which may be found in 62, 208, we informally say that an orbit structure is specified by a portrait $\mathcal{P}$, for example

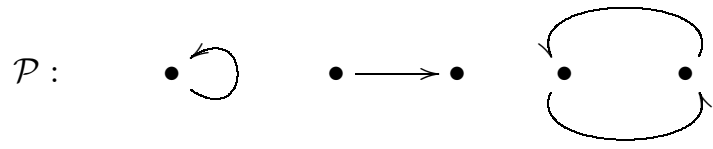

and we define the set $\mathcal{G R} \mathcal{R}_{d}^{1}[\mathcal{P}](K, S)$ to be the subset of an appropriate $\mathcal{G R}_{d}^{1}[n](K, S)$ for which $f: Y \rightarrow X$ models the portrait $\mathcal{P}$. Thus for the portrait (12.2), an element $(f, Y, X)$ of $\mathcal{G} \mathcal{R}_{d}^{1}[\mathcal{P}](K, S)$ consists of a good reduction map $f: \mathbb{P}^{1} \rightarrow \mathbb{P}^{1}$, a set $X$ containing five points that remain distinct modulo all primes not in $S$, and a subset $Y \subset X$ containing four points. Further, the points in $Y$ consist of a fixed 
point of $f$, a 2-cycle for $f$, and a fourth point that $f$ maps to the point in $X$ that is not in $Y$.

Question 12.19. Give criteria, in terms of an integer $d \geq 2$ and the geometry of a portrait $\mathcal{P}$, that distinguish between the following two situations.

(1) $\mathcal{G R}_{d}^{1}[\mathcal{P}](K, S) / \mathrm{PGL}_{2}\left(R_{S}\right)$ is finite for all number fields $K$ and all finite sets of primes $S$.

(2) $\mathcal{G R}_{d}^{1}[\mathcal{P}](K, S) / \mathrm{PGL}_{2}\left(R_{S}\right)$ is infinite for some number fields $K$ and some finite sets of primes $S$.

Remark 12.20. For a given portrait $\mathcal{P}$, one can define a Shafarevich dimension ShafDim ${ }_{d}^{1}[\mathcal{P}]$ associated to $\mathcal{G R}_{d}^{1}[\mathcal{P}]$. Then Questions 12.13 and 12.19 may be generalized by asking for a formula, or algorithm, to compute $\operatorname{ShafDim}_{d}^{1}[\mathcal{P}]$ in terms of $d$ and the geometry of $\mathcal{P}$. See [208] for details, as well as for a complete list of the values of $\operatorname{ShafDim}_{2}^{1}[\mathcal{P}]$ for the 35 portraits that contain at most four points and are allowable for degree 2 maps.

We close this section with another sort of dynamical good reduction problem. In Section 9 we discussed the dynatomic polynomial equation,

$$
Y_{1}^{\mathrm{dyn}}(n): \Phi_{n}(x, z)=0
$$

whose solutions $(\alpha, c)$ characterized points $\alpha$ of formal period $n$ for the quadratic polynomial $f_{c}(x)=x^{2}+c$. The curve $Y_{1}^{\mathrm{dyn}}(n)$ is an irreducible nonsingular plane curve, and as such, we can ask for which primes $p$ does the curve $Y_{1}^{\text {dyn }}(n)$ have bad reduction. Dynamically, this should correspond to some sort of intrinsic collapse among the periodic $n$-cycles of $f_{c}$. By way of analogy, we note that the classical elliptic modular curve $X_{1}(n)$ has bad reduction at exactly the primes dividing $n$, which is quite reasonable, since the $n$-torsion points on an elliptic curve collapse when reduced modulo $p$ if $p$ divides $n$. We might similarly expect that $Y_{1}^{\text {dyn }}(n)$ has bad reduction at the primes dividing $n$, but it also often has bad reduction at other sporadic-looking primes.

Morton [178, defines a discriminant $D_{n}$ whose prime divisors include all primes of bad reduction for $Y_{1}^{\mathrm{dyn}}(n)$, but recent work 60. shows that in many instances, this list is too large. For example, the curve $Y_{1}^{\text {dyn }}(5)$ has bad reduction only at 5 and 3701 , but the set of prime divisors of $D_{5}$ is $\{3,5,11,31,3701,4712,86131\}$. Similarly, although $D_{7}$ has many prime divisors, the dynatomic modular curve $Y_{1}^{\text {dyn }}(7)$ has bad reduction at exactly two primes,

\section{7 and 84562621221359775358188841672549561}

Theorem 12.21 (Doyle-Krieger-Obus-Pries-Rubinstein-Salzedo-West, 2018, [60]). Let $D_{n}$ be Morton's discriminant associated to the nth iterate of $x^{2}+c$; see 60, 178, for the precise definition of $D_{n}$.

(a) Let $n \geq 4$, and let $p$ be an odd prime divisor of $n$. Then $Y_{1}^{\mathrm{dyn}}(n)$ has bad reduction at $p$.

(b) Let $p$ be an odd prime divisor of $D_{n}$ such that $p^{2}$ does not divide $D_{n}$. Then $Y_{1}^{\mathrm{dyn}}(n)$ has bad reduction at $p$.

Theorem 12.21 is a small part of the material in 60], which considers more generally the polynomials $x^{m}+c$, analyzes good and bad reduction of both $Y_{1}^{\text {dyn }}(n)$ 
and $Y_{0}^{\mathrm{dyn}}(n)$, and proves in many cases that $\widetilde{Y_{0}^{\mathrm{dyn}}(n)} \bmod p$ is irreducible. However, we still lack an intrinsic explanation for large sporadic primes of bad reduction.

Question 12.22. Let $Y_{0}^{\mathrm{dyn}}(n)$ and $Y_{1}^{\mathrm{dyn}}(n)$ be the dynamical modular curves classifying points, respectively cycles, of formal period $n$ attached to the family of maps $x^{2}+c$, or $x^{m}+c$, or some other natural one-parameter family of rational maps on $\mathbb{P}^{1}$ such as $b x+x^{-1}$.

(a) Describe the primes of bad reduction for $Y_{0}^{\mathrm{dyn}}(n)$ and $Y_{1}^{\mathrm{dyn}}(n)$.

(b) Give a natural geometric/dynamical explanation for the sporadic primes of bad reduction, which we imprecisely define as primes not dividing $n$ and not dividing a finite set of bad primes associated to the family $f$.

(c) Describe the primes $p$ for which the reductions of $Y_{0}^{\mathrm{dyn}}(n)$ and/or $Y_{1}^{\mathrm{dyn}}(n)$ modulo $p$ are geometrically irreducible, e.g., for the latter, this is asking for the primes $p$ such that the polynomial $\Phi_{n}(x, z)$ does not factor in the $\operatorname{ring} \overline{\mathbb{F}}_{p}[x, z]$.

Note that part of Question 12.22(b) is to precisely define what it means for a bad reduction prime to be sporadic. For example, the results in 60] suggest that for $x^{m}+c$, the sporadic primes for $n$-periodic points are those that do not divide $2 \mathrm{~nm}$.

\section{Dynamical DEgREeS OF RATiOnAL MAPS}

We recall that a rational map $f: \mathbb{P}^{N} \rightarrow \mathbb{P}^{N}$ is described by a list of homogeneous polynomials $f_{0}, f_{1}, \ldots, f_{N} \in K\left[X_{0}, \ldots, X_{N}\right]$ of the same degree, with the proviso that any common factors of $f_{0}, \ldots, f_{N}$ are removed. The degree of $f$ is then the common degree of $f_{0}, \ldots, f_{N}$, and $f$ is dominant if $f_{0}, \ldots, f_{N}$ do not themselves satisfy a nontrivial polynomial relation.

In general, if $\operatorname{deg}(f)=d$, then one might expect that the degree of the $n$th iterate $f^{n}$ would satisfy $\operatorname{deg}\left(f^{n}\right)=d^{n}$. This is true if $f$ is a morphism, and in any case, we always have an inequality $\operatorname{deg}\left(f^{n}\right) \leq d^{n}$, but cancellation of common factors may cause the degree of $f^{n}$ to be smaller than expected.

Example 13.1. The degree 2 rational map $f: \mathbb{P}^{2} \rightarrow \mathbb{P}^{2}$ given by the formula $f(X, Y, Z)=\left[Y Z, X Y, Z^{2}\right]$ has second iterate

$$
f^{2}(X, Y, Z)=f\left(Y Z, X Y, Z^{2}\right)=\left[X Y Z^{2}, X Y^{2} Z, Z^{4}\right]=\left[X Y Z, X Y^{2}, Z^{3}\right] .
$$

Thus $\operatorname{deg}\left(f^{2}\right)=3<4$, since we have canceled a factor of $Z$. An easy induction shows that $\operatorname{deg}\left(f^{n}\right)$ equals the $(n+2)$-nd Fibonacci number.

It is an interesting problem to determine the degree sequence $\operatorname{deg}\left(f^{n}\right)$ of a rational map. The average growth is measured by the following quantity, which was defined independently by Arnold [5], Bellon-Viallet [20, and RussakovskiiShiffman [199.

Definition 13.2. Let $f: \mathbb{P}^{N} \rightarrow \mathbb{P}^{N}$ be a dominant rational map. The dynamical degree of $f$ is

$$
\delta_{f}:=\lim _{n \rightarrow \infty}\left(\operatorname{deg}\left(f^{n}\right)\right)^{1 / n} .
$$

The quantity $\log \left(\delta_{f}\right)$ is sometimes called the algebraic entropy of $f$. 
It is a nice exercise, using the inequality $\operatorname{deg}\left(f^{n+m}\right) \leq \operatorname{deg}\left(f^{n}\right) \operatorname{deg}\left(f^{m}\right)$, to prove that the limit defining $\delta_{f}$ converges. Note that we have $\operatorname{deg}\left(f^{n}\right) \approx \delta_{f}^{n}$ when $n$ is large. As an example, we note that the classical closed formula for Fibonacci numbers implies that the map in Example 13.1 has dynamical degree $\delta_{f}=\frac{1}{2}(1+$ $\sqrt{5}$ ), the so-called Golden Ratio.

Remark 13.3. We recall that a rational map $f=\left[f_{0}, f_{1}, \ldots, f_{N}\right]: \mathbb{P}^{N} \rightarrow \mathbb{P}^{N}$ is a morphism if there are no solutions to $f_{0}=f_{1}=\cdots=f_{N}=0$ in $\mathbb{P}^{N}(\bar{K})$. In this case, it is not hard to see that $\operatorname{deg}\left(f^{n}\right)=\operatorname{deg}(f)^{n}$ for all $n$, and hence $\delta_{f}=\operatorname{deg}(f)$. More generally, a rational map $f$ is said to be algebraically stable if $\operatorname{deg}\left(f^{n}\right)=$ $\operatorname{deg}(f)^{n}$ for all $n$, or equivalently if $\delta_{f}=\operatorname{deg}(f)$.

Based on examples such as Example 13.1, Bellon and Viallet conjectured that the sequence of degrees $\operatorname{deg}\left(f^{n}\right)$ for $n=1,2,3, \ldots$ should satisfy a linear recursion with constant coefficients. This turns out to be false.

Theorem 13.4 (Hasselblatt-Propp, 2007, 106]; Bedford-Kim, 2008, [14]). For every $N \geq 3$ there exist rational maps $f: \mathbb{P}^{N} \rightarrow \mathbb{P}^{N}$ for which the sequence of degrees $\operatorname{deg}\left(f^{n}\right)$ does not satisfy a linear recursion with constant coefficients.

Example 13.5. Hasselblatt and Propp provide the explicit example

$$
f: \mathbb{P}^{3} \rightarrow \mathbb{P}^{3}, \quad f(X, Y, Z, W)=\left[Y W, Z W, X^{2}, X W\right],
$$

whose degree sequence $2,3,4,6,9,12,17,25, \ldots$ does not satisfy a linear recurrence with constant coefficients. However, the construction of Hasselblatt and Propp does not provide a negative answer to the following question.

Question 13.6. Let $f: \mathbb{P}^{N} \rightarrow \mathbb{P}^{N}$ be a dominant rational map. Do there exist a finite collection of constant coefficient linear recursions

$$
L_{n}^{(1)}, L_{n}^{(2)}, \ldots, L_{n}^{(r)}
$$

and an index function $\iota: \mathbb{N} \rightarrow\{1,2, \ldots, r\}$ so that for all $n \geq 1$ we have

$$
\operatorname{deg}\left(f^{n}\right)=L_{n}^{\left(\iota_{n}\right)} ?
$$

And although the original Bellon-Viallet conjecture that the degree sequence is a linear recursion turns out to be false, the following intriguing weaker conjecture is still open.

Conjecture 13.7 (Integrality Conjecture: Bellon-Viallet, 1999, [20]). Let $f$ : $\mathbb{P}^{N} \rightarrow \mathbb{P}^{N}$ be a dominant rational map. Then the dynamical degree $\delta_{f}$ of $f$ is an algebraic integer, i.e., $\delta_{f}$ is the root of a monic polynomial having integer coefficients.

Remark 13.8. Complementing Theorem 13.4, there are nontrivial situations for which it is known that the degree sequence does satisfy a linear recursion. This is true, for example, for birational maps of surfaces (Diller-Favre, 2001, [57]) and for polynomial endomorphsims of $\mathbb{A}^{2}$ (Favre-Jonsson, 2011, 72]). For $\mathbb{P}^{2}$, Conjecture 13.7 is known when $f$ is a birationalm map (Diller-Favre, 2001, [57]) and when $f$ is a polynomial map (Favre-Jonsson, 2007, 71]).

Suppose that the polynomials defining $f: \mathbb{P}^{N} \rightarrow \mathbb{P}^{N}$ have integer coefficients. Then for each prime $p$, we can reduce the coefficients modulo $p$ to create a rational map $f_{p}: \mathbb{P}^{N} \rightarrow \mathbb{P}^{N}$ defined over the finite field $\mathbb{F}_{p}$. For all but finitely many 
primes $p$, the map $f_{p}$ will still be dominant, so we can compute its dynamical degree $\delta_{f_{p}}$. We clearly have $\delta_{f_{p}} \leq \delta_{f}$, but the inequality may be strict, since reducing modulo $p$ may remove some terms that allow us to cancel an extra factor. For example, $f(X, Y)=\left[3 X^{2}+X Y, Y^{2}\right]$ has degree 2, but $f_{3}(X, Y)=\left[X Y, Y^{2}\right]=$ $[X, Y]$ has degree 1 .

Theorem 13.9. (Xie, 2015, 242]) Let $f: \mathbb{P}^{2} \rightarrow \mathbb{P}^{2}$ be a dominant rational map defined by polynomials with integer coefficients. Then

$$
\lim _{p \rightarrow \infty} \delta_{f_{p}}=\delta_{f} .
$$

Lacking evidence, we pose the following as a question, rather than a conjecture.

Question 13.10. Does (13.1) hold for dominant rational maps $f: \mathbb{P}^{N} \rightarrow \mathbb{P}^{N}$ when $N \geq 3$ ?

Example 13.11. Xie [242, Section 5] provides the following example to show that the limit formula (13.1) cannot be strengthened to the statement that $\delta_{f_{p}}=\delta_{f}$ for all sufficiently large $p$. Let

$$
f=\left[X Y, X Y-2 Z^{2}, Y Z+3 Z^{2}\right] .
$$

Then Xie proves that $\delta_{f}=2$, but $\delta_{f_{p}}<2$ for all primes $p$. More precisely, for $p>2$, he shows that $\delta_{f_{p}}$ is the largest real root of the polynomial $\lambda^{\epsilon(p)}-2 \lambda^{\epsilon(p)-1}+1$, where $\epsilon(p)$ is the order of 2 in the multiplicative group $\mathbb{F}_{p}^{*}$.

It is interesting to investigate how dynamical degrees vary in families of maps.

Example 13.12. Consider the three-parameter family of rational maps

$$
f_{a, b, c}([X, Y, Z])=\left[X Y, X Y+a Z^{2}, b Y Z+c Z^{2}\right] .
$$

For most choices of $a, b, c$, we have $\delta_{f_{a, b, c}}=2$, but there are exceptions. It is proven in 222 that for $a, b, c \in \mathbb{C}$, we have $\delta_{f_{a, b, c}}<2$ if and only if there is a root of unity $\xi \in \mathbb{C}$ such that $c^{2}=\left(\xi+\xi^{-1}\right)^{2} a b$.

Conjecture 13.13. Let $f_{T}: \mathbb{P}^{N} \rightarrow \mathbb{P}^{N}$ be a family of dominant rational maps, depending rationally on a list of parameters $T=\left(T_{1}, \ldots, T_{k}\right)$. Then for all $\epsilon>0$, the set

$$
\left\{t \in \mathbb{C}^{k}: \delta\left(f_{t}\right) \leq \delta(f)-\epsilon\right\}
$$

is not Zariski dense in $\mathbb{C}^{k}$. In particular, for one-parameter families, i.e., $k=1$, the set (13.2) is finite.

One approach to proving Conjecture 13.13 would be to prove the following uniform lower bound for $\delta_{f}$, from which it is easy to deduce Conjecture 13.13 . see [222,242].

Conjecture 13.14. Let $N \geq 1$. There exists a constant $\gamma_{N}>0$ such that for all dominant rational maps $f: \mathbb{P}^{N} \rightarrow \mathbb{P}^{N}$, we have

$$
\delta(f) \geq \gamma_{N} \cdot \min _{0 \leq n<N} \frac{\operatorname{deg}\left(f^{n+1}\right)}{\operatorname{deg}\left(f^{n}\right)} .
$$

Note that the constant $\gamma_{N}$ is not allowed to depend on $f$.

Theorem 13.15 (Xie, 2015, 242]). For $N=2$, Conjecture 13.14, and thus also Conjecture 13.13, are true. 
The definition of $\delta_{f}$ says that the degree sequence $\operatorname{deg}\left(f^{n}\right)$ grows roughly like $\delta_{f}^{n}$, but this may not capture the true growth rate. For example, the map $f(X, Y, Z)=$ $\left[X^{d} Y, Y^{d} Z, Z^{d+1}\right]$ has $\delta_{f}=d$ and $\operatorname{deg}\left(f^{n}\right) \sim d^{-1} n \delta_{f}^{n}$. This leads to a refined degree question, posed in varying forms in [106, 220].

Question 13.16. Let $f: \mathbb{P}^{N} \rightarrow \mathbb{P}^{N}$ be a dominant rational map. Is it true that there exists an integer $\ell_{f}$ satisfying $0 \leq \ell_{f} \leq N$ such that 23

$$
\operatorname{deg}\left(f^{n}\right) \asymp n^{\ell_{f}} \delta_{f}^{n},
$$

where the implied constants may depend on $f$, but not on $n$ ?

Question 13.16 has an affirmative answer for monomial maps 76, 154, and for maps of surfaces if $f$ satisfies $\left(\delta_{f}^{(1)}\right)^{2}>\delta_{f}^{(2)}$ (Boucksom-Favre-Jonsson, 2008, [40]). If $f$ is a birational map, then it is known that the sequence of degrees $\operatorname{deg}\left(f^{n}\right)$ is bounded if and only if there is a subsequence of $n$ along which it is bounded, and that if $\operatorname{deg}\left(f^{n}\right)$ is unbounded, then there is a uniform lower bound for its growth rate (Cantat-Xie, 2018, [48]). (This result on birational maps holds more generally for any variety.)

Generalization to algebraic varieties. Let $X$ be a smooth projective variety of dimension $N$, and let $f: X \rightarrow X$ be a dominant rational map. Then $f$ induces a pullback map $f^{*}$ on the group $\operatorname{Pic}(X)$ of line bundles on $X$, but in general it is not true that $\left(f^{n}\right)^{*}$ is the $n$th iterate of $f^{*}$. Letting $\mathcal{L}$ be any ample invertible sheaf on $X$, the dynamical degree of $f$ is defined using intersection theory by the limit

$$
\delta_{f}:=\lim _{n \rightarrow \infty}\left(\left(f^{n}\right)^{*} \mathcal{L} \cdot \mathcal{L}^{N-1}\right)^{1 / n} .
$$

More generally, this $\delta_{f}$ is the first dynamical degree, and for $1 \leq i \leq N$, we define the $i$ th dynamical degree of $f$ by

$$
\delta_{f}^{(i)}:=\lim _{n \rightarrow \infty}\left(\left(\left(f^{n}\right)^{*} \mathcal{L}\right)^{i} \cdot \mathcal{L}^{N-i}\right)^{1 / n} .
$$

It was noted by Guedj 103 that the the sequence of dynamical degrees is concave, i.e., there is a $k$ such that

$$
\delta_{f}^{(1)} \leq \delta_{f}^{(2)} \leq \cdots \leq \delta_{f}^{(k)} \quad \text { and } \quad \delta_{f}^{(k)} \geq \delta_{f}^{(k+1)} \geq \cdots \geq \delta_{f}^{(N)} .
$$

For a description of the full sequence of dynamical degrees when $f$ is a monomial map, see Favre-Wulcan [76] and Lin [155]).

Question 13.17. Let $X$ be a smooth projective variety of dimension $N$, and let $f: X \rightarrow X$ be a dominant rational map. Is it true that the higher order dynamical degrees $\delta_{f}^{(i)}$ are algebraic integers for all $1 \leq i \leq N$ ?

\section{Arithmetic Degrees of orbits}

For a fixed dimension $N$, the degree of a rational map $f: \mathbb{P}^{N} \rightarrow \mathbb{P}^{N}$ is a measure of the complexity of $f$, since for example, it takes on the order of $d^{N}$ coefficients to specify $f$. In an analogous manner, the complexity of a list of integers is measured roughly by the number of bits it takes to specify the integers. The mathematical

\footnotetext{
${ }^{23}$ We recall that the notation $F(n) \asymp G(n)$ means that there are positive constants $c_{1}, c_{2}, c_{3}$, depending on $F$ and $G$, so that $c_{1} F(n) \leq G(n) \leq c_{2} F(n)$ for all $n \geq c_{3}$.
} 
term for this number theoretic complexity is height, which we now describe more precisely. For further details and proofs, see for example [112,143, 214,216.

Definition 14.1. Let $P=\left[a_{0}, a_{1}, \ldots, a_{N}\right] \in \mathbb{P}^{N}(\mathbb{Q})$, where we choose homogeneous coordinates for $P$ satisfying $a_{i} \in \mathbb{Z}$ and $\operatorname{gcd}\left(a_{0}, \ldots, a_{N}\right)=1$. Then the height of $P$ is

$$
h(P):=\log \max \left\{\left|a_{0}\right|,\left|a_{1}\right|, \ldots,\left|a_{N}\right|\right\} .
$$

There is a similar, but more complicated, definition for points $P \in \mathbb{P}^{N}(\overline{\mathbb{Q}})$ whose coordinates are algebraic numbers. But the intuition is that it takes on the order of $h(P)$ bits to describe the point $P$.

It is clear that for any $B$, there are only finitely many $P \in \mathbb{P}^{N}(\mathbb{Q})$ satisfying $h(P) \leq B$, and the same is true of $\mathbb{P}^{N}(K)$ for any number field $K$, and even for the union over all $K$ of a fixed degree over $\mathbb{Q}$. Further, for a rational map $f: \mathbb{P}^{N} \rightarrow \mathbb{P}^{N}$ of degree $d$, an elementary triangle inequality argument shows that there is a constant $A_{f}$ such that

$$
h(f(P)) \leq d \cdot h(P)+A_{f} \quad \text { for all } P \in \mathbb{P}^{N}(\overline{\mathbb{Q}}) \text { with } f(P) \text { defined. }
$$

If $f$ is a morphism, i.e., defined everywhere, a more elaborate argument using the Nullstellensatz can be used to prove that there is a complementary lower bound with another constant $B_{f}$,

$$
h(f(P)) \geq d \cdot h(P)-B_{f} \quad \text { for all } P \in \mathbb{P}^{N}(\overline{\mathbb{Q}}) .
$$

Applying (14.1) repeatedly, we see that the arithmetic complexity, i.e, the height of $f^{n}(P)$, is never much more than a multiple of $d^{n}$. By analogy with the motivation used to define dynamical degree, we measure the average growth rate of the arithmetic complexity of $f^{n}(P)$ by the following quantity, whose logarithm is a sort of arithmetic entropy of the orbit $\mathcal{O}_{f}(P)$.

Definition 14.2. Let $f: \mathbb{P}^{N}(\overline{\mathbb{Q}}) \rightarrow \mathbb{P}^{N}(\overline{\mathbb{Q}})$ be a dominant rational map. We recall that $\mathbb{P}_{f}^{N}(\overline{\mathbb{Q}})$ denotes the set of points $P \in \mathbb{P}^{N}(\overline{\mathbb{Q}})$ for which the entire forward orbit $\mathcal{O}_{f}(P)$ is well-defined. Then the arithmetic degree of the $f$-orbit of a point $P \in$ $\mathbb{P}_{f}^{N}(\overline{\mathbb{Q}})$ was defined in $[220$ ] to be the limit

$$
\alpha_{f}(P):=\lim _{n \rightarrow \infty} h\left(f^{n}(P)\right)^{1 / n} .
$$

Since it is not yet known in general that this limit exists, we also define the upper arithmetic degree using the limsup,

$$
\bar{\alpha}_{f}(P):=\limsup _{n \rightarrow \infty} h\left(f^{n}(P)\right)^{1 / n} .
$$

We note that Theorem 7.9 ensures that $\mathbb{P}_{f}^{N}(\overline{\mathbb{Q}})$ contains lots of wandering points, and for maps $f$ that do not fiber, Conjecture 7.6 says that there will even be lots of points in $\mathbb{P}_{f}^{N}(\overline{\mathbb{Q}})$ having Zariski dense orbits.

We know that $f^{n}$ is given by polynomials of degree roughly $\delta_{f}^{n}$. Combined with (14.1), this suggests that $h\left(f^{n}(P)\right)$ should be no larger than some fixed multiple of $\delta_{f}^{n}$. This is the intuition behind the following inequality, which is proven in [220]:

$$
\bar{\alpha}_{f}(P) \leq \delta_{f} .
$$

(But see the end of this section for the more difficult case when $\mathbb{P}^{N}$ is replaced by a general variety.) The inequality (14.4) quantifies the fact that the arithmetic 
complexity of an orbit is at most equal to the geometric complexity of the iterated map. Part $(\mathrm{d})$ of the next conjecture provides a natural geometric condition that implies equality.

Conjecture 14.3 (Arithmetic Degree Conjectures (on $\mathbb{P}^{N}$ ): Kawaguchi-Silverman, 2006, [134]; see also [136, 137]). Let $f: \mathbb{P}^{N} \rightarrow \mathbb{P}^{N}$ be a dominant rational map defined over $\overline{\mathbb{Q}}$.

(a) Let $P \in \mathbb{P}_{f}^{N}(\overline{\mathbb{Q}})$. The limit (14.3) defining $\alpha_{f}(P)$ exists.

(b) If the map $f$ is not fibered, as described in Definition 7.5, then the set $\left\{\alpha_{f}(P): P \in \mathbb{P}_{f}^{N}(\overline{\mathbb{Q}})\right\}$ is finite.

(c) Let $P \in \mathbb{P}_{f}^{N}(\overline{\mathbb{Q}})$. The arithmetic degree $\alpha_{f}(P)$ is an algebraic integer.

(d) Let $P \in \mathbb{P}_{f}^{N}(\overline{\mathbb{Q}})$ be a point whose orbit $\mathcal{O}_{f}(P)$ is Zariski dense in $\mathbb{P}^{N}$. Then $\alpha_{f}(P)=\delta_{f}$.

Conjecture 14.3 is known for monomial maps, which are maps $f=\left[f_{0}, \ldots, f_{N}\right]$ such that their coordinate functions $f_{i}$ are monomials [220, and parts of the conjecture are known in various other cases; see for example [130, 135, 137.

Remark 14.4. Conjecture14.3(b) applies only to nonfibered maps. This restriction, which is an addendum to the original conjecture, was prompted by the work of Lesieutre and Satriano [149] in which they construct a fibered map $f$ with infinitely many distinct arithmetic degrees $\alpha_{f}(P)$.

We may ask for more refined information regarding the possible growth rates of $h\left(f^{n}(P)\right)$. The following is an arithmetic analogue of Question 13.16

Question 14.5. Let $f: \mathbb{P}^{N} \rightarrow \mathbb{P}^{N}$ be a dominant rational map defined over $\overline{\mathbb{Q}}$, and let $P \in \mathbb{P}_{f}^{N}(\overline{\mathbb{Q}})$ be a point whose orbit $\mathcal{O}_{f}(P)$ is Zariski dense in $\mathbb{P}^{N}$. Is it true that there exist integers $k_{f}$ and $\ell_{f}$ with $0 \leq \ell_{f} \leq N$ such that

$$
h\left(f^{n}(P)\right) \asymp \delta_{f}^{n} \cdot n^{\ell_{f}} \cdot(\log n)^{k_{f}},
$$

where the implied constants depend on $f$ and $P$, but not on $n$ ? Further, if $\delta_{f}>1$, is it true that $k_{f}=0$ ?

If $f: \mathbb{P}^{N} \rightarrow \mathbb{P}^{N}$ is a morphism, then (14.5) holds in the stronger form $h\left(f^{n}(P)\right)=$ $\delta_{f}^{n} \hat{h}_{f}(P)+O(1)$; see Section [15. The situation is more complicated for general varieties. Sano 200 proves that if $f: X \rightarrow X$ is a morphism of a smooth projective variety defined over $\overline{\mathbb{Q}}$, and if $P \in X(\overline{\mathbb{Q}})$ satisfies $\alpha_{f}(P)>1$, then a version of (14.5) holds with $k_{f}=0$.

Example 14.6. To see that the $\log$ term in Question 14.5 may be necessary, consider the map $f: \mathbb{P}^{4} \rightarrow \mathbb{P}^{4}$ given by

$$
f(X, Y, Z, W)=\left[X(Y+Z), W(Y+Z), W Z, W^{2}\right] \quad \text { and } \quad P=[1,0,1,1] .
$$

One can compute $\operatorname{deg}\left(f^{n}\right)=n+1$, so $\delta_{f}=1$ and $\ell_{f}=1$, while $f^{n}(P)=[n !, n, 1,1]$, so $h\left(f^{n}(P)\right) \sim n \log (n)$. See [220, Example 17] for details.

We next seek to quantify the amount by which a rational map may decrease the height of a point. Let $f: \mathbb{P}^{N} \rightarrow \mathbb{P}^{N}$ be a dominant rational map defined over $\overline{\mathbb{Q}}$. The height expansion ratio of $f$ is

$$
\mu(f):=\sup _{\emptyset \neq U \subset \mathbb{P}^{N}} \liminf _{\substack{P \in U(\overline{\mathbb{Q}}) \\ h(P) \rightarrow \infty}} \frac{h(f(P))}{h(P)},
$$


where the sup is over all nonempty Zariski open subsets of $\mathbb{P}^{N}$. Thus $\mu(f)$ measures how much $f$ can reduce the heights of points on a large subset of $\mathbb{P}^{N}$. For example, from (14.1) we see that $\mu(f) \leq \operatorname{deg}(f)$, while (14.2) implies that if $f$ is a morphism, then $\mu(f)=\operatorname{deg}(f)$. It turns out that $\mu(f)$ cannot be arbitrarily small.

Theorem 14.7 (Silverman, 2011, 217]). We have

$$
\bar{\mu}_{d}\left(\mathbb{P}^{N}\right):=\inf _{\substack{f: \mathbb{P}^{N} \rightarrow \mathbb{P}^{N} \\ \operatorname{dominant} \\ \operatorname{deg}(f)=d}} \mu(f)>0 .
$$

It is known that $\bar{\mu}_{d}\left(\mathbb{P}^{N}\right) \leq d^{-(N-1)}$ for all $N \geq 2$ and all $d \geq 2$; see [217. Proposition 10] and [146]. This leads to the obvious question.

Question 14.8. What is the exact value of $\bar{\mu}_{d}\left(\mathbb{P}^{N}\right)$ ?

Generalization to algebraic varieties. In order to generalize Conjecture 14.3 to a projective variety $X$, we need an appropriate height function.

Definition 14.9. Let $X$ be a smooth projective variety, let $i: X \hookrightarrow \mathbb{P}^{N}$ be an embedding, and let $f: X \rightarrow X$ be a dominant rational map, with everything defined over $\overline{\mathbb{Q}}$. Then the arithmetic degree of the $f$-orbit of $P \in X_{f}(\overline{\mathbb{Q}})$ is

$$
\alpha_{f}(P):=\lim _{n \rightarrow \infty} h\left(i \circ f^{n}(P)\right)^{1 / n} .
$$

We write $\bar{\alpha}_{f}(P)$ for (14.6) with the limit replaced by limsup.

It is not hard to verify that $\alpha_{f}(P)$, if it exists, does not depend on the embedding $X$ in projective space. The main conjectures on arithmetic degrees carry over verbatim to the general case.

Conjecture 14.10 (Arithmetic Degree Conjectures: Kawaguchi-Silverman, 2006, 134, 136, 137]). Let $f: X \rightarrow X$ be a dominant rational map of a smooth projective variety $X$ defined over $\overline{\mathbb{Q}}$.

(a) Let $P \in X_{f}(\overline{\mathbb{Q}})$. The limit (14.6) defining $\alpha_{f}(P)$ exists.

(b) If the map $f$ is not fibered, as described in Definition 7.5, then the set $\left\{\alpha_{f}(P): P \in X_{f}(\overline{\mathbb{Q}})\right\}$ is finite.

(c) Let $P \in X_{f}(\overline{\mathbb{Q}})$. The arithmetic degree $\alpha_{f}(P)$ is an algebraic integer.

(d) Let $P \in X_{f}(\overline{\mathbb{Q}})$ whose orbit $\mathcal{O}_{f}(P)$ is Zariski dense in $X$. Then $\alpha_{f}(P)=\delta_{f}$.

The inequality

$$
\bar{\alpha}_{f}(P) \leq \delta_{f} \quad \text { for } P \in X_{f}(\overline{\mathbb{Q}})
$$

relating arithmetic and dynamical degrees is true in the more general setting of Conjecture 14.10, but the proof is no longer as elementary as it was for $X=\mathbb{P}^{N}$, even if we assume that $f$ is a morphism. See 136 for a proof of (14.7) when $f$ is a morphism, and see [167] for the general case of dominant rational maps.

Progress on Conjecture 14.10 is fragmentary. Parts (a), (b), and (c) are true when $f$ is a morphism [136]. The more difficult part (d) has been proven when $f$ is a monomial map [136, when $X$ is an abelian variety, i.e., when $X$ has the structure of an algebraic group [136, 221], and when $f$ is a morphism and either $X$ is surface [168, or $X$ is higher dimensional with additional structure [148. 


\section{CAnOnical heights}

We recall from Section 14 that the height $h(P)$ of a point $P \in \mathbb{P}^{N}(\overline{\mathbb{Q}})$ measures the arithmetic complexity of $P$, and that a degree $d$ morphism $f: \mathbb{P}^{N} \rightarrow \mathbb{P}^{N}$ has the effect, roughly, of multiplying the height by $d$. More precisely,

$$
h(f(P))=d h(P)+O(1) \text { for all } P \in \mathbb{P}^{N}(\overline{\mathbb{Q}}),
$$

where the big- $O$ bound depends on $f$, but is independent of $P$. This suggests that $h\left(f^{n}(P)\right)$ grows like $d^{n}$, and in Section 14 we took the limit of $h\left(f^{n}(P)\right)^{1 / n}$ to measure this growth. When $f$ is a morphism, we can do much better.

Definition 15.1. Let $f: \mathbb{P}^{N} \rightarrow \mathbb{P}^{N}$ be a morphism of degree $d$ defined over $\overline{\mathbb{Q}}$, and let $P \in \mathbb{P}^{N}(\overline{\mathbb{Q}})$. The (dynamical) canonical height of $P$ for the map $f$ is the quantity

$$
\hat{h}_{f}(P):=\lim _{n \rightarrow \infty} \frac{1}{d^{n}} h\left(f^{n}(P)\right) .
$$

The definition of $\hat{h}_{f}$ is modeled after Tate's construction of the canonical (NéronTate) height on abelian varieties. See [214, §3.4] for the telescoping sum proof that the limit (15.1) exists.

The dynamical canonical height has several agreeable properties:

$$
\begin{aligned}
\hat{h}_{f}(f(P)) & =d \hat{h}_{f}(P) . \\
\hat{h}_{f}(P) & =h(P)+O(1) . \\
\hat{h}_{f}(P)=0 & \Longleftrightarrow P \in \operatorname{PrePer}(f) .
\end{aligned}
$$

We note that $\hat{h}_{f}$ is uniquely determined by (15.2) and (15.3).

The classical Lehmer conjecture gives a lower bound for the height of algebraic numbers that are not roots of unity. It is the $N=1$ and $f(x)=x^{2}$ case of the following conjecture, which provides a quantitative converse to (15.4).

Conjecture 15.2 (Dynamical Lehmer Conjecture). Let $f: \mathbb{P}^{N} \rightarrow \mathbb{P}^{N}$ be a morphism of degree $d \geq 2$ defined over a number field $K$. There is a constant $C(f)>0$ such that for all $P \in \mathbb{P}^{N}(\overline{\mathbb{Q}})$ that are wandering for $f$, we hav 24

$$
\hat{h}_{f}(P) \geq \frac{C(f)}{[K(P): K]} .
$$

Temporarily writing $D_{K}(P):=[K(P): K]$, Dobrowolski [58, proved a lower bound of the form $\hat{h}_{f}(P) \geq C(f, \epsilon) D_{K}(P)^{-1}\left(\log \log D_{K}(P) / \log D_{K}(P)\right)^{6+\epsilon}$, and Laurent 145 proved a similar result for Lattès maps that have CM. For non-CM Lattès maps, Masser [166] proved a result of the form $\hat{h}_{f}(P) \geq C(f, \epsilon) D_{K}(P)^{3+\epsilon}$. However, as far as the authors are aware, if $f(x) \in K(x)$ is not associated to an endomorphism of an algebraic group, there are no lower bounds for $\hat{h}_{f}(P)$ that decay slower than exponentially in $D_{K}(P)$.

For maps on $\mathbb{P}^{1}$ associated endomorphisms of algebraic groups, e.g., $f(x)=x^{d}$ or a Lattès map, there are lower bounds for $\hat{h}_{f}(P)$ that decay.

In order to state the next conjecture, we need a way to measure the arithmetic complexity of a rational map $f: \mathbb{P}^{N} \rightarrow \mathbb{P}^{N}$ defined over $\overline{\mathbb{Q}}$. It is not enough to simply use the coefficients of the polynomials defining $f$, since as we saw in

\footnotetext{
${ }^{24}$ We recall from Section 3 that $K(P)$ is the field generated by the coordinates of $P$.
} 
Section [10, changing coordinates on $\mathbb{P}^{N}$ has the effect of drastically changing the coefficients of $f$ without affecting the underlying dynamical system. The solution is to view $f$ as a point $\langle f\rangle$ in $\mathcal{M}_{d}^{N}$, the space of maps up to change-of-coordinates. The space $\mathcal{M}_{d}^{N}$ is itself an algebraic variety, so we fix an embedding

$$
\iota: \mathcal{M}_{d}^{N} \longleftrightarrow \mathbb{P}^{L},
$$

and then we define the moduli height of $f$ to be the height of the associated point in projective space,

$$
h_{\mathcal{M}}(f):=h(\iota(\langle f\rangle)) .
$$

One can show that any two such heights are commensurable, in the sense that if $h_{\mathcal{M}}^{\prime}$ corresponds to some other embedding $\iota^{\prime}$ of $\mathcal{M}_{d}^{N}$, then there are positive constants $c_{1}, c_{2}, c_{3}, c_{4}$ such that

$$
c_{1} h_{\mathcal{M}}(f)-c_{2} \leq h_{\mathcal{M}}^{\prime}(f) \leq c_{3} h_{\mathcal{M}}(f)+c_{4} \quad \text { for all } f \in \mathcal{M}_{d}^{N}(\overline{\mathbb{Q}}) .
$$

Informally, the next conjecture says that the arithmetic complexity of a wandering orbit is always larger than the arithmetic complexity of the map. It is a generalization of a conjecture that Serge Lang formulated for elliptic curves [142], and it provides a different quantitative converse to (15.4).

Conjecture 15.3 (Dynamical Lang Height Conjecture). Let $N \geq 1, D \geq 1$, and $d \geq 2$. There are positive constants $c_{1}(D, N, d)$ and $c_{2}(D, N, d)$ such that for all number fields $K / \mathbb{Q}$ with $[K: \mathbb{Q}] \leq D$, all degree d morphisms $f: \mathbb{P}^{N} \rightarrow \mathbb{P}^{N}$ defined over $K$, and all points $P \in \mathbb{P}^{N}(K)$ whose orbit $\mathcal{O}_{f}(P)$ is Zariski dense in $\mathbb{P}^{N}$, we have

$$
\hat{h}_{f}(P) \geq c_{1}(D, N, d) h_{\mathcal{M}}(f)+c_{2}(D, N, d) .
$$

See [34, 35] for numerical evidence supporting Conjecture 15.3 in the case $N=$ $D=1$ and $d \in\{2,3\}$, including conjectural values for the constants. We also note that the Zariski density assumption in Conjecture 15.3 is necessary. For example, the map $f=\left[X^{2}, Y^{2}, \gamma Z^{2}\right]$ has height roughly $h(\gamma)$, while the $f$-orbit of the point $P=[\alpha, 1,0]$ has canonical height $\hat{h}_{f}(P)=h(\alpha)$, so we can make $h_{\mathcal{M}}(f)$ arbitrarily large while $\hat{h}_{f}(P)$ remains bounded. Ingram [118, and Looper [159] have proven versions of Conjecture 15.3 for polynomial maps $f$ of $\mathbb{P}^{1}$, where the constants depend also on the number of primes of bad reduction of $f$.

The following generalization of Conjecture 11.3 is a dynamical version of a conjecture of Bogomolov concerning points of small height on subvarieties of tori and abelian varieties. Bogomolov's original conjectures were proven by Ullmo [238, and Zhang 245.

Conjecture 15.4 (Dynamical Bogomolov Conjecture). Let $T, a, b$, and $f$ be maps as in (11.1) for some $d \geq 2$, with all maps defined over $\overline{\mathbb{Q}}$. Then the following are equivalent.

(a) For every $\epsilon>0$, the set $\left\{t \in T(\overline{\mathbb{Q}}): \hat{h}_{f_{t}}\left(a_{t}\right) \leq \epsilon\right.$ and $\left.\hat{h}_{f_{t}}\left(b_{t}\right) \leq \epsilon\right\}$ is infinite.

(b) The maps $a$ and $b$ are $f$-dynamically related.

The dynamics of a rational map $f: \mathbb{P}^{1} \rightarrow \mathbb{P}^{1}$ is greatly influenced by the orbits of its critical points. We write $\operatorname{Crit}(f)$ for the set of critical points of $f$ in $\mathbb{P}^{1}$, counted with appropriate multiplicities. 
Definition 15.5. Let $f: \mathbb{P}^{1} \rightarrow \mathbb{P}^{1}$ be a rational map of degree at least 2 defined over $\overline{\mathbb{Q}}$. The critical height of $f$ is

$$
\hat{h}^{\text {crit }}(f):=\sum_{P \in \operatorname{Crit}(f)} \hat{h}_{f}(P) .
$$

We observe from (15.4) that $\hat{h}^{\text {crit }}(f)=0$ if and only if every critical point of $f$ is preperiodic, i.e., if and only if $f$ is PCF. In order to state the next result, which provides an interpretation of $\hat{h}^{\text {crit }}$ and was conjectured by Silverman circa 2010, we need a somewhat technical definition. However, the only part of the definition that is required in order to understand Theorem 15.7 is that most maps are not Lattès maps.

Definition 15.6. For each integer $m$ and each elliptic curve $E / \mathbb{C}$, we construct a rational map $f_{E, m}$ by choosing a Weierstrass equation $y^{2}=x^{3}+A x+B$ for $E$ and requiring $f_{E, m}$ to satisfy the equation

$$
f_{E, m}(x(P))=x(m P) \quad \text { for all } P \in E(\mathbb{C}) .
$$

The map $f_{E, m}$ has degree $m^{2}$ and is called a (flexible) Lattès map. The collection of all Lattès maps in $\mathcal{M}_{m^{2}}^{1}$ forms a one-dimensional family, so most maps are not Lattès maps, since $\operatorname{dim} \mathcal{M}_{d}^{1}=2 d-2$. Lattès maps have many special properties; in particular, every Lattès map is PCF. For further information about Lattès maps, see for example [176] or [214, Chapter 6].

Theorem 15.7. (Ingram, 2016, 121) Let $d \geq 2$. There are positive constants $c_{1}, c_{2}, c_{3}, c_{4}$, depending only on $d$, such that for all non-Lattès degree $d$ rational maps $f: \mathbb{P}^{1} \rightarrow \mathbb{P}^{1}$ defined over $\overline{\mathbb{Q}}$, we have

$$
c_{1} h_{\mathcal{M}}(f)-c_{2} \leq \hat{h}^{\mathrm{crit}}(f) \leq c_{3} h_{\mathcal{M}}(f)+c_{4} .
$$

We remark that the easier upper bound part of Theorem 15.7 had been proven earlier by Silverman [218, Theorem 6.31]. We also note that an important corollary of Theorem 15.7 is that outside of the Lattès locus, the set of PCF maps in $\mathcal{M}_{d}^{1}$ is a set of bounded height. This weaker result had been proven earlier by Benedetto, Ingram, Jones, and Levy [22]. See also [84 for a recent quantitative version of Theorem 15.7

Question 15.8. Is there a natural generalization of Theorem 15.7 for morphisms $f: \mathbb{P}^{N} \rightarrow \mathbb{P}^{N}$ ? The moduli height $h_{\mathcal{M}}$ was defined earlier for all $\mathcal{M}_{d}^{N}$, but it is not at all clear what should take the place of the critical height when $N \geq 2$.

Generalization to algebraic varieties. In this subsection we assume that the reader is familiar with various standard terms from algebraic geometry.

Let $X / \overline{\mathbb{Q}}$ be a smooth projective variety, let $f: X \rightarrow X$ be a $\overline{\mathbb{Q}}$-morphism, and let $D \in \operatorname{Div}_{\overline{\mathbb{Q}}}(X) \otimes \mathbb{R}$ be an $\mathbb{R}$-linear sum of divisors on $X$. One can associate to the divisor class of $D$ an equivalence class of height functions $h_{D}: X(\overline{\mathbb{Q}}) \rightarrow \mathbb{R}$, well-defined up to bounded functions; see for example [112,143].

Definition 15.9. Suppose that there is a real number $\lambda>1$ with the property that

$$
f^{*} D \sim \lambda D \quad \text { in } \operatorname{Pic}_{\overline{\mathbb{Q}}}(X) \otimes \mathbb{R}
$$


Then the dynamical canonical height of $P$ for the map $f$ and divisor (class) $D$ is the quantity

$$
\hat{h}_{f, D}(P):=\lim _{n \rightarrow \infty} \frac{1}{\lambda^{n}} h\left(f^{n}(P)\right) .
$$

It is known that the limit (15.6) exists [46], and that it satisfies

$$
\hat{h}_{f, D}(f(P))=\lambda \hat{h}_{f, D}(P) \text { and } \hat{h}_{f, D}(P)=h_{D}(P)+O(1) .
$$

Further,

$$
D \text { is ample } \Longrightarrow \hat{h}_{f, D}(P) \geq 0 \text { and } \quad \hat{h}_{f, D}(P)=0 \Longleftrightarrow P \in \operatorname{PrePer}(f) .
$$

Unfortunately, not every map admits an ample divisor satisfying $f^{*} D \sim \lambda D$, but we can find a nice divisor if we relax the ampleness condition to instead allow $D$ to be numerically effective (nef), and we relax the linear equivalence (15.5) to be an algebraic equivalence.

Proposition 15.10. Let $f: X \rightarrow X$ be a finite endomorphism of a smooth variety, let $\delta_{f}$ be the associated dynamical degree, and suppose that $\delta_{f}>1$.

(a) [137, Remark 29] There exists a nonzero nef divisor class $\theta_{f} \in \mathrm{NS}(X) \otimes \mathbb{R}$ with the property that

$$
f^{*} \theta_{f} \equiv \delta_{f} \theta_{f} \quad \text { in } \mathrm{NS}(X) \otimes \mathbb{R} .
$$

(b) [137, Theorem 5] Let $\theta_{f} \in \mathrm{NS}(X) \otimes \mathbb{R}$ be as in (a). Then the canonical height limit (15.6) with $D \equiv \theta_{f}$ and $\lambda=\delta_{f}$ exists. Denoting the limit by $\hat{h}_{f, \theta_{f}}(P)$, for any ample divisor $H$ on $X$, this nef canonical height satisfies

$$
\hat{h}_{f, \theta_{f}} \circ f=\delta_{f} \hat{h}_{f, \theta_{f}} \quad \text { and } \quad \hat{h}_{f, \theta_{f}}=h_{\theta_{f}}+O\left(\sqrt{h_{H}}\right) .
$$

Question 15.11. Let $X, f, \theta_{f}$, and $\hat{h}_{f, \theta_{f}}$ be as in Proposition 15.10.

(a) Is it true that $\hat{h}_{f, \theta_{f}}(P) \geq 0$ for all $P \in X(\overline{\mathbb{Q}})$ ?

(b) Describe the set of points

$$
\left\{P \in X(\overline{\mathbb{Q}}): \hat{h}_{f, \theta_{f}}(P) \leq 0\right\},
$$

beyond the obvious fact that it contains $\operatorname{PrePer}(f)$.

Remark 15.12. Here are three cases where the answer to Question 15.11 is known.

(1) If $\theta_{f}$ is ample, then it is easy to see that $\hat{h}_{f, \theta_{f}} \geq 0$ and that the set (15.8) equals $\operatorname{PrePer}(f)$.

(2) Let $X$ be an abelian variety, and let $f$ be an isogeny. Then $\hat{h}_{f, \theta_{f}} \geq 0$, and there is a proper abelian subvariety $Y \subsetneq X$ such that the set (15.8) in (b) is equal to the set of translates $Y+X_{\text {tors }}$; see [136.

(3) Let $X$ be a K3 surface with $\operatorname{rank} \mathrm{NS}(X)=2$, and let $f: X \rightarrow X$ be an automorphism of infinite order obtained by composing noncommuting involutions. Then $\hat{h}_{f, \theta_{f}} \geq 0$, and the set (15.8) equals PrePer $(f)$; see [209].

Even less is known for rational maps that are not morphisms. The following was suggested by Mattias Jonsson.

\footnotetext{
${ }^{25}$ A divisor is nef (numerically effective) if it lies in the closure of the ample cone in $\mathrm{NS}(X) \otimes \mathbb{R}$. Equivalently, the divisor $D$ is nef if for every ample divisor $H$ and every $n \geq 1$, the divisor $n D+H$ is ample. Thus, nonample nef divisors are on the edge of being ample.
} 
Question 15.13. Let $X$ be a smooth projective variety of dimension $N$, let $f$ : $X \rightarrow X$ be a dominant rational map, and suppose that the higher order dynamical degrees $\delta_{f}^{(1)}, \ldots, \delta_{f}^{(N)}$ defined earlier (13.3) form a strictly log concave sequence, i.e., they satisfy $\left(\delta_{f}^{(i)}\right)^{2}>\delta_{f}^{(i-1)} \delta_{f}^{(i+1)}$ for all $1 \leq i \leq N-1$. Also let $D$ be an ample divisor.

(a) Is it true that $\operatorname{deg}_{D}\left(f^{n}\right):=\left(f^{n}\right)^{*} D \cdot D^{N-1} \asymp\left(\delta_{f}^{(1)}\right)^{n}$ ?

(b) Suppose that $X, f$, and $D$ are defined over $\overline{\mathbb{Q}}$, and let $P \in X_{f}(\overline{\mathbb{Q}})$. Does the limit

$$
\hat{h}_{f, D}(P):=\lim _{n \rightarrow \infty}\left(\delta_{f}^{(1)}\right)^{-n} h_{D}\left(f^{n}(P)\right)
$$

converge? If so, is it independent of $D$ up to a multiplicative constant?

(c) Assuming that the limit in (b) exists, describe the set of $P \in X(\overline{\mathbb{Q}})$ satisfying $\hat{h}_{f, D}(P)=0$, beyond the fact that it contains $\operatorname{PrePer}(f)$.

For $\operatorname{dim}(X)=2$, the answer to Question 15.13(a) is yes [40, and for birational $f$ there are partial results for (b) and (c) in [129].

\section{VARIATION OF THE CANONICAL HEIGHT}

Conjecture 15.3 asks for the smallest positive value of the canonical height $\hat{h}_{f}(P)$ as $f$ varies over all degree $d$ morphisms $f: \mathbb{P}^{N} \rightarrow \mathbb{P}^{N}$ and $P$ varies over all $f$ wandering points. Questions about the variation of the canonical height become easier if we require that the maps and points vary in an algebraic family. Thus let $B$ be a quasi-projective variety, let $f_{t}: \mathbb{P}^{N} \rightarrow \mathbb{P}^{N}$ be a family of morphisms parameterized by $t \in B$, and let $P: B \rightarrow \mathbb{P}^{N}$ be a morphism, so we may view $P_{t}$ as being a family of points parameterized by $B$. It is then natural to ask about the behavior of the function

$$
B(\bar{K}) \longrightarrow \mathbb{R}, \quad t \longmapsto \hat{h}_{f_{t}}\left(P_{t}\right),
$$

or to relate it to other natural functions on $B$.

Alternatively, we may view $f$ as an endomorphism of $\mathbb{P}^{N}$ defined over the function field $K(B)$ of $B$, and then $P$ is a point in $\mathbb{P}^{N}(K(B))$. In the case that $B$ is a curve, each element of $\beta \in K(B)$ defines a map $\beta: B \rightarrow \mathbb{P}^{1}$. We define the height $h(\beta)$ to be the degree of the map $\beta$. We can similarly define the height of a point $P \in \mathbb{P}^{N}(K(B))$, and by taking limits, we obtain a canonical height $\hat{h}_{f}(P)$ for $P$ relative to the map $f$. We also define a normalized height on $B(\overline{\mathbb{Q}})$ by fixing a nonconstant rational map $\beta: B \rightarrow \mathbb{P}^{1}$ and defining

$$
h_{B}: B(\overline{\mathbb{Q}}) \longrightarrow \mathbb{R}, \quad h_{B}(t):=\frac{1}{\operatorname{deg} \beta} h(\beta(t)) .
$$

More generally, for every divisor $D \in \operatorname{Div}(B) \otimes \mathbb{R}$, one can define an associated Weil height function $h_{B, D}: B(\overline{\mathbb{Q}}) \rightarrow \mathbb{R}$, well-defined up to $O(1)$; see [112,143, for details.

Generalizing an earlier result of Silverman for families of abelian varieties, Call and Silverman proved the following result relating three different height functions associated to a one-dimensional family of maps and points.

Theorem 16.1 (Call-Silverman, 1993, [46, Theorem 4.1]). Let $B$ be a smooth curve, and let $f: \mathbb{P}^{N} \rightarrow \mathbb{P}^{N}$ and $P: B \rightarrow \mathbb{P}^{N}$ be a family of maps and a family of 
points parameterized by $B$. Then

$$
\lim _{\substack{t \in B(\overline{\mathbb{Q}}) \\ h_{B}(t) \rightarrow \infty}} \frac{\hat{h}_{f_{t}}\left(P_{t}\right)}{h_{B}(t)}=\hat{h}_{f}(P) .
$$

Theorem 16.1 is an asymptotic formula for a ratio of heights. To what extent can it be strengthened? If $f$ is a family of Lattès maps, Tate 235] proved that one can associate to $f$ and $P$ a divisor $E_{f, P} \in \operatorname{Div}(B) \otimes \mathbb{Q}$ such that

$$
\hat{h}_{f_{t}}\left(P_{t}\right)=h_{B, E_{f, P}}(t)+O(1) .
$$

Thus the map $t \mapsto \hat{h}_{f_{t}}\left(P_{t}\right)$ is a Weil height on the base curve $B$. In particular, if $B \cong \mathbb{P}^{1}$, then any two divisors of the same degree give the same height, up to $O(1)$, so in this case Tate's result for Lattès maps implies that

$$
\hat{h}_{f_{t}}\left(P_{t}\right)=\hat{h}_{f}(P) h(t)+O(1) .
$$

For an arbitrary base curve $B$, there is a weaker estimate in which the $O(1)$ is replaced by $O\left(\sqrt{h_{B}(t)}\right)$.

In general, let $f_{t}: \mathbb{P}^{N} \rightarrow \mathbb{P}^{N}$ be a family of morphisms parameterized by a nonsingular variety $B$, and let $P: B \rightarrow \mathbb{P}^{N}$ be a morphism giving a family of points parameterized by $B$. We define a divisor associated to $f$ and $P$ by choosing a hyperplane $H$ in $\mathbb{P}^{N}$ and taking the limit

$$
E_{f, P}:=\lim _{n \rightarrow \infty} \frac{1}{d^{n}}\left(f^{n} \circ P\right)^{*} H \in \operatorname{Div}(B) \otimes \mathbb{R} .
$$

The limit should exist, with the height $h_{B, E}$ on $B$ associated to $E$ not depending on the choice of $H$, up to the usual $O(1)$.

Conjecture 16.2. Let $f_{t}: \mathbb{P}^{N} \rightarrow \mathbb{P}^{N}$ be a family of morphisms parameterized by a nonsingular curve $B$, let $P: B \rightarrow \mathbb{P}^{N}$ be a morphism, and let $E_{f, B}$ be the divisor defined by (16.2). Then

$$
\hat{h}_{f_{t}}\left(P_{t}\right)=h_{B, E_{f, B}}(t)+O(1) \text { as } t \text { ranges over } B(\bar{K}) \text {. }
$$

More generally, one might extend Conjecture 16.2 to a base $B$ of higher dimension, noting that there is no guarantee that $E_{f, B}$ will be ample, and indeed, in the case of constant families it is the zero divisor.

Some progress on Conjecture 16.2 has been made, including for the following type of map.

Definition 16.3. A morphism $f: \mathbb{P}^{N} \rightarrow \mathbb{P}^{N}$ is a regular polynomial endomorphism (RPE) if there is a hyperplane $H \subset \mathbb{P}^{N}$ satisfying $f^{-1}(H)=H$. Equivalently, let $f_{0}: \mathbb{A}^{N} \rightarrow \mathbb{A}^{N}$ be a polynomial map whose extension $f: \mathbb{P}^{N} \rightarrow \mathbb{P}^{N}$ is a morphism. Then $f$ is an RPE.

A hyperplane $H \subset \mathbb{P}^{N}$ is isomorphic to $\mathbb{P}^{N-1}$, so restricting an RPE $f$ to its invariant hyperplane $H$ gives a morphism $\tilde{f}: \mathbb{P}^{N-1} \rightarrow \mathbb{P}^{N-1}$, and thus defines a point $\langle\tilde{f}\rangle \in \mathcal{M}_{d}^{N-1}$. We say that a family $f_{t}: \mathbb{P}^{N} \rightarrow \mathbb{P}^{N}$ of RPEs parameterized by $t \in B$ is fibral if $\left\langle\tilde{f}_{t}\right\rangle$ is constant as $t$ varies over the parameter space $B$. For example, all polynomials $f(x) \in \bar{K}[x]$ are RPEs of $\mathbb{P}^{1}$, and in this case one can show that every family of RPEs is fibral. 
Theorem 16.4 (Ingram [19, 120]). Conjecture 16.2 is true for fibral families of regular polynomial endomorphisms of $\mathbb{P}^{N}$, and in this case the divisor $E_{f, P}$ is in $\operatorname{Div}(B) \otimes \mathbb{Q}$.

Although the restriction to fibral families may seem strong, it is noted in 120 that extending Theorem 16.4 to arbitrary families of RPEs would be enough to extend it to arbitrary families of endomorphisms.

For a more refined conjecture, we can decompose the canonical height $\hat{h}_{f}$ associated to a morphism $f: \mathbb{P}^{N} \rightarrow \mathbb{P}^{N}$ into a sum of canonical local heights,

$$
\hat{h}_{f}=\sum_{v} \hat{\lambda}_{f, v}
$$

where the sum is over equivalence classes of absolute values $v$ on the field $K$, and $\hat{\lambda}_{f, v}$ is a function $\mathbb{P}^{N}\left(K_{v}\right) \rightarrow \mathbb{R} \cup\{\infty\}$ on the points of $\mathbb{P}^{N}$ defined over the $v$-completion $K_{v}$ of $K$. Roughly speaking, the local canonical height of a point $P \in \mathbb{P}^{N}\left(K_{v}\right)$ is characterized by

(16.3) $\hat{\lambda}_{f, v}(P)=-\log (v$-adic distance from $P$ to the hyperplane at $\infty)+O(1)$

and a transformation formula for the difference $\hat{\lambda}_{f, v}(f(P))-(\operatorname{deg} f) \hat{\lambda}_{f, v}(P)$. See [46] for details.

Question 16.5. Given a family of maps $f: \mathbb{P}^{N} \rightarrow \mathbb{P}^{N}$ and a family of points $P$, parameterized by $B$, describe the map

$$
B\left(K_{v}\right) \longrightarrow \mathbb{R} \cup\{\infty\}, \quad t \longmapsto \hat{\lambda}_{f_{t}, v}\left(P_{t}\right) .
$$

In particular, to what extent is it a Weil function associated to some divisor in $\operatorname{Div}(B) \otimes \mathbb{R}$ ?

The following result gives progress on this problem, contingent on a technical condition.

Theorem 16.6 (Mavraki-Ye [169]). Let $f_{t}: \mathbb{P}^{1} \rightarrow \mathbb{P}^{1}$ be a family of morphisms parameterized by a nonsingular curve $B \cong \mathbb{P}^{1}$, let $P: B \rightarrow \mathbb{P}^{1}$ be a morphism, and let $D$ be a divisor of degree 1 on $B$. Suppose further that the pair $(f, P)$ is quasi-adelic. Then

$$
\hat{h}_{f_{t}}\left(P_{t}\right)=\hat{h}_{f}(P) h_{B, D}(t)+O(1) .
$$

We refer the reader to [169] for the somewhat technical definition of quasi-adelic, which has to do with the continuity, or more generally summability, properties of certain canonical measures on Berkovich $\mathbb{P}^{1}$.

Conjecture 16.2 suggests another natural question. It turns out that in Tate's formula (16.1) and in Theorem 16.4, the divisor $E_{f, P} \in \operatorname{Div}(B) \otimes \mathbb{R}$ is in fact an element of $\operatorname{Div}(B) \otimes \mathbb{Q}$. The former follows from the existence of the Néron model for elliptic curves, the latter from the existence of Böttcher coordinates for polynomial endomorphisms. These imply in these cases that the canonical height $\hat{h}_{f}(P)$ of $P$ over the function field of $B$ is a rational number.

Question 16.7. Let $K(B)$ be the function field of an algebraic curve defined over an algebraically closed field $K$ of characteristic 0 , let $f: \mathbb{P}^{N} \rightarrow \mathbb{P}^{N}$ be a morphism of degree $d \geq 2$ defined over the field $K(B)$, and let $P \in \mathbb{P}^{N}(K(B))$. Is the canonical height $\hat{h}_{f}(P)$ always in $\mathbb{Q}$ ? 
Question 16.7 is open, even for $N=1$, but it may be contrasted with the following result for local canonical heights over the completion of $K(B)$.

Theorem 16.8 (DeMarco-Ghioca [54]). Let $K(B)$ be as in Question 16.7, let $v$ be a valuation of $K(B)$, let $K(B)_{v}$ be a completion of $K(B)$ at $v$, and let $\widehat{K(B)}$, be a minimal completion of an algebraic closure of $K(B)_{v}$, so in particular $v\left(\widehat{K(B)_{v}}\right)=$ $\mathbb{Q}$. Then for every $d \geq 2$, there exists a rational function $f: \mathbb{P}^{1} \rightarrow \mathbb{P}^{1}$ of degree $d$ and a point $P \in \mathbb{P}^{1}\left(\widehat{K(B)_{v}}\right)$ such that the local canonical height $\hat{\lambda}_{f, v}(P)$ is irrational.

We note that the value of the local canonical height depends on various choices, but the statement of Theorem 16.8 is independent of these choices. We also note that Theorem 16.8 is a result about points defined over $\widehat{K(B)}$, so it does not seem helpful for studying Question 16.7, which is about points defined over $K(B)$. We will see a similar dichotomy in the next section when we discuss non-archimedean wandering domains; cf. Theorems 17.4 and 17.5 and Conjecture 17.6.

\section{7. $p$-ADIC AND NON-ARCHIMEDEAN DYNAMICS}

As noted in Section 1, the study of dynamics over global fields leads naturally to questions about dynamics over local fields. In addition, the rich theory of complex dynamics on the one hand provides a model for a theory of $p$-adic dynamics, and on the other hand raises the question of how much of the archimedean theory generalizes to non-archimedean fields. Moreover, besides $p$-adic fields, there are questions in complex dynamics whose solutions use dynamics over Laurent series fields such as $\mathbb{C}((t))$, which are non-archimedean, but not $p$-adic. Thus, we consider the following general setting.

Let $\mathbb{C}_{v}$ be an algebraically closed field that is complete with respect to an absolute value $|\cdot|_{v}$ satisfying the non-archimedean triangle inequality,

$$
|a+b|_{v} \leq \max \left\{|a|_{v},|b|_{v}\right\} \text { for all } a, b \in \mathbb{C}_{v} .
$$

For example, for any prime number $p$, the field $\mathbb{C}_{p}$ is the completion of an algebraic closure of the $p$-adic field $\mathbb{Q}_{p}$. We set the additional notation

$$
\mathcal{O}_{v}:=\left\{a \in \mathbb{C}_{v}:|a|_{v} \leq 1\right\} \text { and } \mathcal{M}_{v}:=\left\{a \in \mathbb{C}_{v}:|a|_{v}<1\right\}
$$

for, respectively, the ring of integers and the maximal ideal for $\mathbb{C}_{v}$.

The projective line $\mathbb{P}^{1}\left(\mathbb{C}_{v}\right)$ over $\mathbb{C}_{v}$ is totally disconnected and not locally compact, but it can be embedded in the Berkovich projective line $\mathrm{P}_{\text {Berk }}^{1}$, which is a compact, Hausdorff, and path-connected topological space that contains $\mathbb{P}^{1}\left(\mathbb{C}_{v}\right)$ as a dense subset. We briefly describe some of the salient features of $P_{\text {Berk }}^{1}$ and its endomorphisms, and we refer the reader to [11, Chapters 1-2], [33, Chapters 6-7], or [214, Section 5.10] for further details.

The points of $\mathrm{P}_{\text {Berk }}^{1}$ that lie in $\mathbb{P}^{1}\left(\mathbb{C}_{v}\right)$ are said to be of Type I. The points of Type II and III in $\mathrm{P}_{\text {Berk }}^{1}$ correspond to closed disks in $\mathbb{C}_{v}$. Type II points correspond to rational disks, which are disks whose radius is in $\left|\mathbb{C}_{v}^{\times}\right|_{v}$, while Type III points correspond to irrational disks, which are disks whose radius is not in $\left|\mathbb{C}_{v}^{\times}\right|_{v}$. Finally, there are Type IV points that will play only a minor role in this survey 26 The Type II point $\zeta(0,1)$ corresponding to the closed unit disk $\bar{D}(0,1) \subset \mathbb{C}_{v}$ is called the Gauss point of $\mathrm{P}_{\text {Berk }}^{1}$.

\footnotetext{
${ }^{26}$ Type IV points correspond to decreasing chains $D_{1} \supset D_{2} \supset \cdots$ of disks in $\mathbb{C}_{v}$ with empty intersection. They are needed to ensure that $\mathrm{P}_{\text {Berk }}^{1}$ is compact.
} 
A rational function $f(z) \in \mathbb{C}_{v}(z)$ defines a map $f: \mathbb{P}^{1}\left(\mathbb{C}_{v}\right) \rightarrow \mathbb{P}^{1}\left(\mathbb{C}_{v}\right)$, and this map extends continuously to a function

$$
f: \mathrm{P}_{\text {Berk }}^{1} \rightarrow \mathrm{P}_{\text {Berk }}^{1}
$$

Each point $\zeta \in \mathrm{P}_{\text {Berk }}^{1}$ maps to its image $f(\zeta)$ with a local degree, or multiplicity, which is an integer between 1 and $\operatorname{deg}(f)$. If the local degree of $f$ at $\zeta$ is divisible by the residue characteristic of $\mathbb{C}_{v}$, we say that $\zeta$ is wild.

For a Type I point $\zeta$, the image $f(\zeta)$ is defined using the map $f: \mathbb{P}^{1}\left(\mathbb{C}_{v}\right) \rightarrow$ $\mathbb{P}^{1}\left(\mathbb{C}_{v}\right)$, and the local degree at $\zeta$ is the usual multiplicity (or ramification index) of $f$ at $\zeta$. To describe how $f$ acts on points of Types II and III, we note that if $D_{1} \subset \mathbb{C}_{v}$ is a disk, then the image $f\left(D_{1}\right)$ is either a disk $D_{2} \subset \mathbb{C}_{v}$, the complement $\mathbb{P}^{1}\left(\mathbb{C}_{v}\right) \backslash D_{2}$ of a disk, or all of $\mathbb{P}^{1}\left(\mathbb{C}_{v}\right)$. In the first two cases, the action of $f$ on $\mathrm{P}_{\text {Berk }}^{1}$ maps the point $\zeta_{1} \in \mathrm{P}_{\text {Berk }}^{1}$ corresponding to $D_{1}$ to the point $\zeta_{2} \in \mathrm{P}_{\text {Berk }}^{1}$ corresponding to $D_{2}$. In particular, if $D_{1}$ and $D_{2}$ are closed disks with $D_{2}=f\left(D_{1}\right)$, then the local degree of $f$ at $\zeta_{1}$ is $\# f^{-1}(\zeta)$ for any point $\zeta \in D_{2}$.

Let $f(z) \in \mathbb{C}_{v}(z)$, and let $a \in \mathbb{P}^{1}\left(\mathbb{C}_{v}\right)$ be an $f$-periodic point of exact period $n$. For ease of exposition, we assume that $\infty \notin \mathcal{O}_{f}(a) 27$ Then the multiplier of $f$ at a is the quantity

$$
\lambda_{f}(a)=\left(f^{n}\right)^{\prime}(a)=\prod_{i=0}^{n-1} f^{\prime}\left(f^{i}(a)\right) .
$$

We say that $a$ is

- attracting if $\left|\lambda_{f}(a)\right|_{v}<1$,

- indifferent, or neutral, if $\left|\lambda_{f}(a)\right|_{v}=1$, or

- repelling if $\left|\lambda_{f}(a)\right|_{v}>1$.

Periodic points of Types II, III, and IV have multipliers that are positive integers; more precisely, the multiplier of such a periodic point $\zeta$ is defined to be the local degree of $f^{n}$ at $\zeta$, where $n$ is the period of $\zeta$ under $f$. Periodic points of Types III and IV necessarily have multiplier 1 , because any rational function fixing a point $\zeta$ of Type III or IV must act as a homeomorphism on a neighborhood of $\zeta$.

The multiplier of a Type II point is more interesting and is computed as follows. Suppose that $\zeta=\zeta(a, r)$ is the point corresponding to the rational closed disk $\bar{D}(a, r)$, where $a \in \mathbb{C}_{v}$ and $r \in\left|\mathbb{C}_{v}^{\times}\right|_{v}$. Replacing $f$ by $\phi^{-1} \circ f \circ \phi$ for an appropriate $\phi(z)=c z+a$, we may assume $\zeta=\zeta(0,1)$ is the Gauss point. Write $f^{n}=g / h$ as a quotient of relatively prime polynomials $g(z), h(z) \in \mathcal{O}_{v}[z]$ such that at least one coefficient $u$ of $g$ or $h$ satisfies $|u|_{v}=1$. Then we can reduce the coefficients of $g$ and $h$ modulo $\mathcal{M}_{v}$ to obtain a rational function $\bar{g}(z) / \bar{h}(z) \in\left(\mathcal{O}_{v} / \mathcal{M}_{v}\right)(z)$ with coefficients in the residue field. The assumption that $f^{n}$ fixes the Gauss point implies that $\bar{g} / \bar{h}$ is nonconstant, and then the multiplier of $f$ at $\zeta$ is the degree of the rational function $\bar{g} / \bar{h}$. We say that a periodic point $\zeta$ of Type II, III, or IV is indifferent if its multiplier is 1 and is repelling if its multiplier is at least 2. (Points of Types II, III, and IV are never attracting.)

\footnotetext{
${ }^{27}$ The multiplier $\lambda_{f}(a)$ is invariant under change of coordinates, so if $\infty \in \mathcal{O}_{f}(a)$, we can compute $\lambda_{f}(a)$ by first changing coordinates.
} 
The (Berkovich) Fatou set of $f(z) \in \mathbb{C}_{v}(z)$ is the set $\mathcal{F}_{f}$ of those points $\zeta \in \mathrm{P}_{\text {Berk }}^{1}$ which have a neighborhood $U$ such that

$$
\mathrm{P}_{\text {Berk }}^{1} \backslash\left(\bigcup_{n \geq 0} f^{n}(U)\right) \quad \text { is infinite. }
$$

The (Berkovich) Julia set of $f$ is the complement $\mathcal{J}_{f}=\mathrm{P}_{\text {Berk }}^{1} \backslash \mathcal{F}_{f}$. For alternative equivalent definitions, see for example [11, Section 10.5]. The Fatou and Julia sets are both invariant under $f$, i.e.,

$$
f\left(\mathcal{F}_{f}\right)=f^{-1}\left(\mathcal{F}_{f}\right)=\mathcal{F}_{f} \quad \text { and } \quad f\left(\mathcal{J}_{f}\right)=f^{-1}\left(\mathcal{J}_{f}\right)=\mathcal{J}_{f}
$$

For any $n \geq 1$, the Fatou set of $f^{n}$ coincides with the Fatou set of $f$, and similarly for the Julia set.

All repelling periodic points lie in the Julia set, while the Fatou set contains all attracting periodic points, all indifferent periodic points of Types I, III, and IV, and most of the Type II indifferent points. However, if the residue field of $\mathbb{C}_{v}$ is not algebraic over a finite field, e.g., if $\mathbb{C}_{v}$ has residue characteristic 0 , then some Type II indifferent periodic points may lie in the Julia set. By way of contrast, in classical complex dynamics, most indifferent periodic points lie in the Julia set. The Berkovich Fatou set is always nonempty because $f$ necessarily has a nonrepelling fixed point of Type I; see [26, Corollary 1.3]. The Julia set is also nonempty because $f$ necessarily has a repelling fixed point of Type I or II; see [197, Théorème B]. However, the Julia set may contain no Type I points, i.e., it is possible to have $\mathcal{J}_{f} \cap \mathbb{P}^{1}\left(\mathbb{C}_{v}\right)=\emptyset$. This happens, for example, if $f$ has good reduction; cf. Section 12 .

It is true that the repelling periodic points are dense in the Julia set 33 . Section 12.3], but if we restrict our attention to $\mathbb{P}^{1}\left(\mathbb{C}_{v}\right)$, i.e., to the Type I points, then things are more complicated. We pose a conjecture.

Conjecture 17.1. Let $f(z) \in \mathbb{C}_{v}(z)$ be a rational function of degree at least 2 , and let $\mathcal{J}_{f} \subset \mathrm{P}_{\text {Berk }}^{1}$ be the Julia set of $f$. Then $\mathcal{J}_{f} \cap \mathbb{P}^{1}\left(\mathbb{C}_{v}\right)$ is equal to the closure in $\mathbb{P}^{1}\left(\mathbb{C}_{v}\right)$ of the set of Type I repelling periodic points of $f$.

Hsia 113 proved that $\mathcal{J}_{f} \cap \mathbb{P}^{1}\left(\mathbb{C}_{v}\right)$ is contained in the closure of all Type I periodic points, but in contrast to the situation over $\mathbb{C}$, a rational map over $\mathbb{C}_{v}$ may have infinitely many nonrepelling periodic points. On the other hand, Bézivin [37] proved that if $f$ has at least one Type I repelling periodic point, then Conjecture 17.1 is true.

Let $U$ be a connected component of $\mathcal{F}_{f}$. Then the image $f(U)$ is also a connected component of $\mathcal{F}_{f}$. Further, there is an integer $m \in\{1, \ldots, d\}$ such that every point in $f(U)$ has exactly $m$ preimages in $U$, counted with multiplicity; we say that $f$ is $m$-to- 1 on $U$. A periodic component $U$ of period $n \geq 1$ is said to be

- attracting if $f^{n}$ is $m$-to- 1 on $U$ with $m \geq 2$;

- indifferent if $f^{n}$ is 1-to-1 on $U$.

Rivera-Letelier proved the following classification theorem over $\mathbb{C}_{p}$, and his proof can be extended to arbitrary $\mathbb{C}_{v}$ [33. Chapter 9]. To state it, we define a domain 
of Cantor type 28 to be an increasing union $\bigcup_{j \geq 1} U_{j}$, where each $U_{j}$ is the complement in $\mathrm{P}_{\text {Berk }}^{1}$ of finitely many disjoint closed Berkovich disks $V_{j, i}$, with each $V_{j, i}$ containing at least two of the disks $V_{j+1, \ell}$.

Theorem 17.2 (Rivera-Letelier, 2000, [196]). Let $f(z) \in \mathbb{C}_{v}(z)$ be a rational function of degree at least 2, and let $U \subseteq \mathcal{F}_{f}$ be a periodic Fatou component of period $n \geq 1$.

(a) If $U$ is an attracting component, then there is a Type I attracting periodic point $x \in \mathcal{F}_{f}$ such that all points of $U$ are attracted to $x$ under iteration of $f^{n}$. Moreover, $U$ is either a rational open disk or a domain of Cantor type.

(b) If $U$ is an indifferent component, then $U$ is a region of the form

$$
U=\mathrm{P}_{\text {Berk }}^{1} \backslash\left(V_{1} \cup \cdots \cup V_{N}\right),
$$

where $V_{1}, \ldots, V_{N}$ are disjoint rational closed disks. The $N$ boundary points of $U$ are all periodic under $f^{n}$.

In the same paper, Rivera-Letelier proved that a map $f(z) \in \mathbb{C}_{v}(z)$ of degree $d \geq$ 2 can have at most $d-1$ cycles of attracting periodic components that are of Cantor type. However, if there are wild points $\zeta \in \mathrm{P}_{\text {Berk }}^{1}$, i.e., points at which the local degree of $f$ is divisible by the residue characteristic, then there may be infinitely many attracting components that are disks. For example, the Gauss point is fixed and wild for the map $f(z)=z^{p} \in \mathbb{C}_{p}(z)$, and every periodic residue class $D(a, 1)$ is an attracting component. By contrast, if there are no wild points in $\mathrm{P}_{\text {Berk }}^{1}$, for example if the residue characteristic is 0 or strictly greater than $d$, then there are at most $2 d-2$ cycles of attracting components; see [22, Corollary 1.6].

There can also be infinitely many indifferent periodic disks. For example, if $f(z)=z^{d}$, and if the residue characteristic $p$ does not divide $d$, then every periodic residue class $D(a, 1)$, besides the ones at 0 and $\infty$, is indifferent. Rivera-Letelier showed that $f$ can have many cycles of nondisk indifferent components 196. Proposition 6.4], but his construction increases the degree of $f$ as it increases the number of such cycles, suggesting the following question.

Question 17.3. Let $f(z) \in \mathbb{C}_{v}(z)$ with $\operatorname{deg} f=d \geq 2$. Can $f$ have infinitely many periodic cycles of indifferent components that are not disks? If not, is there a bound in terms of $d$ for the number of such cycles? Do the answers depend on the field $\mathbb{C}_{v}$ ?

A component of $\mathcal{F}_{f}$ that is not preperiodic is said to be a wandering domain. A famous theorem of Sullivan 230] in complex dynamics says that the Fatou set of a rational function $f(z) \in \mathbb{C}(z)$ has no wandering domains in $\mathbb{P}^{1}(\mathbb{C})$. The nonarchimedean situation is more complicated. The following result first appeared for $p$-adic fields, but its proof generalizes to function fields of positive characteristic under stronger hypotheses. To state it, we say that a point $b$ is recurrent under $f$ if $b$ is itself an accumulation point of its forward orbit $\mathcal{O}_{f}(b)$.

Theorem 17.4 (Benedetto, 2000, 25]). Let $K$ be a locally compact subfield of $\mathbb{C}_{v}$, and suppose that the algebraic closure $\bar{K}$ of $K$ is dense in $\mathbb{C}_{v}$. Let $p \geq 2$ be the

\footnotetext{
${ }^{28}$ The name comes from the fact that the boundary of such a domain is homeomorphic to the Cantor set.
} 
residue characteristic of $\mathbb{C}_{v}$, and let $f(z) \in K(z)$ be a rational function of degree at least 2. Suppose that one of the following two conditions holds.

(a) char $K=0$, and there are no wild recurrent critical points of Type $I$ in $\mathcal{J}_{f}$.

(b) char $K=p$, and there are no wild critical points of Type I in $\mathcal{J}_{f}$.

Then $f$ has no wandering domains.

The residue field of a locally compact non-archimedean field is necessarily finite. Thus Theorem 17.4(a) applies to $p$-adic fields, and Theorem 17.4(b) applies to function fields over finite fields. However, if $f(z) \in \mathbb{C}_{v}(z)$ is not required to be defined over a locally compact subfield of $\mathbb{C}_{v}$, then $f$ may have wandering domains, as in the following explicit result. The construction relies heavily on both wild behavior, i.e., maps that are locally $m$-to- 1 with $p \mid m$, and the fact that $\mathbb{C}_{v}$ is not locally compact.

Theorem 17.5 (Benedetto, 2002 and 2006, 28,30). Suppose that $\mathbb{C}_{v}$ has residue characteristic $p \geq 2$. Then for any $b \in \mathbb{C}_{v}$ with $|b|_{v}>1$ and any $\epsilon>0$, there exists $a \in \mathbb{C}_{v}$ with $|a-b|_{v}<\epsilon$ such that the Fatou set of the polynomial

$$
f(z)=(1-a) z^{p+1}+a z^{p}
$$

has a wandering domain.

The contrasting conclusions of Theorems 17.4 and 17.5 suggest the following conjecture.

Conjecture 17.6 ([25]). Let $K$ be a locally compact subfield of $\mathbb{C}_{v}$, and suppose that the algebraic closure $\bar{K}$ of $K$ is dense in $\mathbb{C}_{v}$. Let $f(z) \in K(z)$ be a rational function of degree at least 2 . Then $f$ has no wandering domains.

In contrast to the locally compact case, if the residue field of $\mathbb{C}_{v}$ is not an algebraic extension of a finite field, then it turns out that there are wandering domains attached to every periodic point of Type II. For example, the map $f(z)=$ $z^{d}$ fixes the Gauss point, and every open disk of the form $U=D(b, 1)$ with $|b|=1$ satisfies $\phi^{n}(U)=D\left(b^{d^{n}}, 1\right)$. If $\mathbb{C}_{v}$ is of residue characteristic 0 , then some such disks $U$ contain no roots of unity, e.g., for $b=2$, and in fact, $U$ is a wandering domain of $\mathcal{F}_{f}$. Such wandering domains are said to lie in the attracting basin of the associated (Julia) periodic point of Type II. However, it appears that no other wandering domains are possible in residue characteristic 0.

Theorem 17.7. Let $f(z) \in \mathbb{C}_{v}(z)$ be a rational function of degree $d \geq 2$, and let $p$ be the residue characteristic of $\mathbb{C}_{v}$. Suppose that at least one of the following conditions holds.

(a) (Benedetto, 2005, 29, Theorem B]) The function $f$ is defined over a discretely valued subfield $K$ of $\mathbb{C}_{v}$ whose algebraic closure $\bar{K}$ is dense in $\mathbb{C}_{v}$, and either $p=0$ or $p>d$.

(b) (Trucco, 2014, 237]) The function $f$ is a polynomial, i.e., $f(z) \in \mathbb{C}_{v}[z]$, and $p=0$.

Then every wandering domain of $f$ lies in the attracting basin of a Type II Julia periodic point.

The condition in Theorem 17.7 (a) that $\bar{K}$ is dense in $\mathbb{C}_{v}$ cannot be removed, because if $\left|\bar{K}^{\times}\right|_{v} \subsetneq\left|\mathbb{C}_{v}^{\times}\right|_{v}$, then any Lattès map arising from an elliptic curve 
having multiplicative reduction will have wandering domains defined over $\mathbb{C}_{v}$, but which correspond to Type III points over the completion of $\bar{K}$.

The conditions on the residue characteristic in Theorem 17.7 are used mainly to eliminate the possibility of wild behavior. Thus we are led to the following conjecture, which applies to maps defined over the $p$-adic field $\mathbb{C}_{p}$ and maps defined over non-archimedean function fields of arbitrary characteristic.

Conjecture 17.8. Let $K \subset \mathbb{C}_{v}$ be a discretely valued subfield whose algebraic closure $\bar{K}$ is dense in $\mathbb{C}_{v}$. Let $p \geq 0$ be the residue characteristic of $\mathbb{C}_{v}$, and let $f(z) \in \mathbb{C}_{v}(z)$ be a rational function of degree at least 2 . Suppose that there are no points $\zeta \in \mathrm{P}_{\text {Berk }}^{1}$ at which $f$ has local degree divisible by $p$. Then every wandering domain of $f$ lies in the attracting basin of a Type II Julia periodic point.

Remark 17.9. Another important tool used in studying non-archimedean dynamics is measure theory. Given $f(z) \in \mathbb{C}_{v}(z)$, there is a naturally associated Borel probability measure $\mu_{f}$ on $\mathrm{P}_{\text {Berk }}^{1}$ called the canonical measure or invariant measure or equilibrium measure for $f$. Constructed independently by Baker and Rumely [10, Chambert-Loir [50], and Favre and Rivera-Letelier [73, 74], the measure $\mu_{f}$ has many useful properties. In particular, its support is precisely the Julia set $\mathcal{J}_{f}$, and it is invariant under $f$, i.e., $\mu_{f}\left(f^{-1}(E)\right)=\mu_{f}(E)$ for every Borel subset $E$ of $\mathrm{P}_{\text {Berk }}^{1}$. The existence of $\mu_{f}$ permits a meaningful study of ergodic theory, Lyapunov exponents, and more, in the context of non-archimedean dynamics. In addition, if $f$ is defined over a global field $K$, then the measures $\mu_{f, v}$ attached to the different absolute values $v$ on $K$ are closely related to the local canonical height functions (16.3) discussed in Section 15. A deeper discussion of the equilibrium measure and open questions surrounding it would unfortunately take us too far afield in this survey; we refer the interested reader to [11, Section 10.1] or [33, Chapter 13] for more information.

Remark 17.10. As mentioned in the introduction, there is a thriving study of ergodic theory and entropy over $p$-adic fields dating back to at least a 1975 paper of Oselies and Zieschang [185]. For recent work on $p$-adic ergodic theory for rational maps, see the paper [75] of Favre and Rivera-Letelier, and for a discussion of $p$-adic entropy, see the paper [156] of Lind and Ward.

\section{DyNAMICS OVER FINITE FIELDS}

Projective space $\mathbb{P}^{N}\left(\mathbb{F}_{q}\right)$ over a finite field $\mathbb{F}_{q}$ contains only finitely many points, precisely $\# \mathbb{P}^{N}\left(\mathbb{F}_{q}\right)=\left(q^{N+1}-1\right) /(q-1)$, so a morphism $f: \mathbb{P}^{N} \rightarrow \mathbb{P}^{N}$ defined over $\mathbb{F}_{q}$ is a self-map of the finite set $\mathbb{P}^{N}\left(\mathbb{F}_{q}\right)$. Thus every point is preperiodic, but it is interesting to study the subset of points that are periodic.

Definition 18.1. Let $S$ be a (finite) set. We write

$$
\begin{aligned}
& \operatorname{End}_{\text {Set }}(S)=\text { the collection of set maps } S \rightarrow S \text {, } \\
& \operatorname{Autset}_{\text {( }}(S)=\text { the collection of set bijections } S \rightarrow S \text {, } \\
& \operatorname{Per}(f, S)=\text { the set of periodic points for } f \in \operatorname{End}_{\text {Set }}(S) \\
& =\left\{P \in S: f^{n}(P)=P \text { for some } n \geq 1\right\} \text {. }
\end{aligned}
$$

We note that a map $f \in \operatorname{End}_{\text {Set }}(S)$ gives $S$ the structure of a directed graph $\Gamma_{f}$ whose vertices are the element of $S$ and such that there is an arrow from $P$ to $f(P)$ for each $P \in S$. 
If we let $f$ range over $\operatorname{End}_{\text {Set }}(S)$ or $\operatorname{Aut}_{\text {Set }}(S)$ and let $\# S \rightarrow \infty$, then there are known statistics for \# $\operatorname{Per}(f, S)$ and for various natural quantities associated to the graph $\Gamma_{f}$ such as its number of components, its largest component, its largest cycle, and many others. See for example [80, 105, 141]. In particular, Flajolet and Odlyzko [80] analyze a large number of properties for average random maps, and we may ask whether similar statistics hold if we restrict our inquiry to maps defined by polynomials.

Question 18.2 (Vague Motivating Question). To what extent do polynomial maps behave like random set maps?

In this section we briefly discuss some potential answers to this question, where we note that there are many sorts of averages that one might consider. For example, we can average over maps defined over a given field $\mathbb{F}_{q}$, or we can fix a map defined over $\mathbb{F}_{q}$ and ask what happens to the points in $\mathbb{P}^{N}\left(\mathbb{F}_{q^{n}}\right)$ as $n \rightarrow \infty$, or we can fix a map defined over $\mathbb{Q}$ and average over its $\bmod p$ reductions.

It is important to note that the collection of degree $d$ morphisms $f: \mathbb{P}^{N}\left(\mathbb{F}_{q}\right) \rightarrow$ $\mathbb{P}^{N}\left(\mathbb{F}_{q}\right)$ is generally only a small part of the collection of set maps $\operatorname{End}_{\text {Set }}\left(\mathbb{P}^{N}\left(\mathbb{F}_{q}\right)\right)$. For example, the inverse image of a point $P \in \mathbb{P}^{N}\left(\mathbb{F}_{q}\right)$ satisfies $\# f^{-1}(P) \leq \operatorname{deg}(f)$, and further, for a given $1 \leq k \leq \operatorname{deg}(f)$, the "probability" that $\# f^{-1}(P)=k$ may depend on $k$ in subtle ways; see [19].

We have already discussed in Section 12 the situation where we start with a morphism $f: \mathbb{P}^{N} \rightarrow \mathbb{P}^{N}$ defined over $\mathbb{Q}$ and a point $P \in \mathbb{P}^{N}(\mathbb{Q})$, and we reduce modulo various primes $p$ and study the orbit of the reduced point $\tilde{P}$ for the reduced map $\tilde{f}$. In particular, Propostion [12.5 says that iteration and reduction modulo $p$ interact nicely with one another for all but finitely many primes $p$. If $P$ is $f$ wandering in $\mathbb{P}^{N}(\mathbb{Q})$, it is interesting to ask how $\mathcal{O}_{\tilde{f}}(\tilde{P} \bmod p)$ varies as we vary the prime $p$. For example, is it usually large, as predicted by the following conjecture 29 where we refer the reader to Definition 3.1 for the definition of the (natural) density of a set of primes.

\section{Conjecture 18.3.}

(a) Let $f: \mathbb{P}^{N} \rightarrow \mathbb{P}^{N}$ be a morphism of degree $d \geq 2$ defined over $\mathbb{Q}$, and let $P \in \mathbb{P}^{N}(\mathbb{Q})$ be a point whose $f$-orbit $\mathcal{O}_{f}(P)$ is Zariski dense in $\mathbb{P}^{N}$. Then for every $\epsilon>0$, the set of primes

$$
\left\{p: \# \mathcal{O}_{\tilde{f}}(\tilde{P} \bmod p) \geq p^{(1 / 2-\epsilon) N}\right\}
$$

is a set of density 1 .

(b) Let $f: \mathbb{A}^{N} \rightarrow \mathbb{A}^{N}$ be a polynomial automorphism defined over $\mathbb{Q}$, i.e., the map $f$ is defined by polynomials, and it has an inverse defined by polynomials. Let $P \in \mathbb{A}^{N}(\mathbb{Q})$ be a point whose f-orbit $\mathcal{O}_{f}(P)$ is Zariski dense in $\mathbb{A}^{N}$. Then for every $\epsilon>0$, the set of primes

$$
\left\{p: \# \mathcal{O}_{\tilde{f}}(\tilde{P} \bmod p) \geq p^{(1-\epsilon) N}\right\}
$$

is a set of density 1.

Only much weaker estimates are known. Here is a typical result; see also [51,215] for generalizations involving parameterized families of collections of maps.

\footnotetext{
${ }^{29}$ This conjecture should probably be viewed as a provocation.
} 
Theorem 18.4. [1, Akbary-Ghioca (2009)] Let $\epsilon>0$.

(a) Let $f: \mathbb{P}^{N} \rightarrow \mathbb{P}^{N}$ be an endomorphism defined over $\mathbb{Q}$. Then the set of primes

$$
\left\{p: \# \mathcal{O}_{\tilde{f}}(\tilde{P} \bmod p) \geq \frac{\log (p)}{\operatorname{deg}(f)+\epsilon}\right\}
$$

is a set of density 1 .

(b) Let $f: \mathbb{A}^{N} \rightarrow \mathbb{A}^{N}$ be a polynomial automorphism defined over $\mathbb{Q}$. Then the set of primes

$$
\left\{p: \# \mathcal{O}_{\tilde{f}}(\tilde{P} \bmod p) \geq p^{1 / 2-\epsilon}\right\}
$$

is a set of density 1 .

In the context of Conjecture 18.3(a), one might ask instead for a density 1 conclusion for primes whose orbits are smaller than $p^{(1 / 2+\epsilon) N}$. As explained in [107, it seems unlikely that such a result holds in general, so we pose the problem of characterizing those maps for which there is such a result.

Question 18.5. Let $f: \mathbb{P}^{N} \rightarrow \mathbb{P}^{N}$ be a morphism of degree $d \geq 2$ defined over $\mathbb{Q}$, and let $P \in \mathbb{P}^{N}(\mathbb{Q})$ be a point whose $f$-orbit $\mathcal{O}_{f}(P)$ is Zariski dense in $\mathbb{P}^{N}$. Under what conditions on $f$ is it true that for every $\epsilon>0$, the set of primes

$$
\left\{p: \# \mathcal{O}_{\tilde{f}}(\tilde{P} \bmod p) \leq p^{(1 / 2+\epsilon) N}\right\}
$$

is a set of density 1 ?

There are a handful of published results giving upper bounds for the size of the orbit $\mathcal{O}_{\tilde{f}}(\tilde{P} \bmod p)$, including [107, 131,206]. To illustrate, we quote an easily stated corollary from one of these papers.

Theorem 18.6 (Heath-Brown, 2017, [107]). Let $f(x)=a x^{2}+b$ with $a, b \in \mathbb{Z}$ positive integers. There is a constant $C(a, b)$ such that for every $\alpha \in \mathbb{Z}$ and every odd prime $p$,

$$
\# \mathcal{O}_{\tilde{f}}(\tilde{\alpha} \bmod p) \leq C(a, b) \frac{p}{\log \log p} .
$$

We next consider the graph $\Gamma_{f}$ induced by the action of $f$ on the points of $\mathbb{P}^{N}$. For any graph $\Gamma$, we write

$$
\operatorname{Comp}(\Gamma)=\# \text { of components in the graph } \Gamma \text {. }
$$

Kruskal [141] proved that the average value of $\operatorname{Comp}(\Gamma)$ for random maps of a set $S$ with $n$ elements is given by

$$
\operatorname{Comp}_{\text {avg }}(n):=\frac{1}{n^{n}} \sum_{f \in \operatorname{Endset~}_{\text {set }}(S)} \operatorname{Comp}\left(\Gamma_{f}\right)=\frac{1}{2} \log n+C+o(1),
$$

with an explicit value for $C$. This suggests a natural question.

Question 18.7. Fix an integer $N \geq 1$ and a prime power $q$. Is it true that

$$
\frac{1}{\# \operatorname{End}_{d}^{N}\left(\mathbb{F}_{q}\right)} \sum_{f \in \operatorname{End}_{d}^{N}\left(\mathbb{F}_{q}\right)} \operatorname{Comp}\left(\Gamma_{f}\right)=\operatorname{Comp}_{\text {avg }}\left(\# \mathbb{P}^{N}\left(\mathbb{F}_{q}\right)\right)+O(1) \quad \text { as } d \rightarrow \infty \text { ? }
$$


One could, in Question 18.7, ask for a stronger result with $o(1)$, or for a weaker result with $\sim$ or $\asymp$ in place of $=$. And rather than taking endomorphisms of $\mathbb{P}^{N}$, one could instead average over endomorphisms of $\mathbb{A}^{N}$, i.e., over polynomial self-maps of $\mathbb{F}_{q}^{N}$.

Flynn and Garton 82 prove that if $N=1$ and $d \geq \sqrt{q}$, then the average in Question 18.7 is greater than $\frac{1}{2} q-4$. See also [19] for an affirmative answer to Question 18.7 for $N=1$ assuming an independence hypothesis. For material on the connectivity of the graphs $\Gamma_{x^{2}+c}$ as $c$ ranges over $\mathbb{F}_{q}$, see [138]; and for results on the number of nonisomorphic graphs $\Gamma_{f}$ as $f$ ranges over polynomials in $\mathbb{F}_{q}[x]$ of fixed degree, see 164 .

Rather than averaging over all maps in $\operatorname{End}_{d}^{N}\left(\mathbb{F}_{q}\right)$, we might instead fix a map defined over a number field and look at the components of the graphs that we obtain by reducing modulo different primes. This leads to the following question.

Question 18.8. Let $f: \mathbb{P}^{N} \rightarrow \mathbb{P}^{N}$ be a morphism of degree $d \geq 2$ defined over $\mathbb{Q}$. Is it true that for every $\epsilon>0$, the set of primes

$$
\left\{p: \operatorname{Comp}\left(\Gamma_{\tilde{f} \bmod p}\right) \leq \operatorname{Comp}_{\text {avg }}\left(p^{(1-\epsilon) N}\right)\right\}
$$

is a set of density 0 ? Similarly with $\mathbb{A}^{N}$ in place of $\mathbb{P}^{N}$ ? For which maps $f$ can one obtain stronger results, e.g., $\operatorname{Comp}\left(\Gamma_{\tilde{f} \bmod p}\right) \asymp \operatorname{Comp}_{\text {avg }}\left(p^{N}\right)$ as $p \rightarrow \infty$ ?

For our next problem, we start with an (irreducible) polynomial $f(x) \in \mathbb{F}_{q}[x]$ and ask how the iterates $f^{n}(x)$ factor in $\mathbb{F}_{q}[x]$. It turns out to be useful to introduce a supplementary polynomial $g(x) \in \mathbb{F}_{q}[x]$ and study the factorization of $g\left(f^{n}(x)\right)$. In this generality, very little is known, or even specifically conjectured, so our next question is necessarily somewhat vague.

Question 18.9. Let $f(x) \in \mathbb{F}_{q}[x]$ be an (irreducible) polynomial, let $g(x) \in \mathbb{F}_{q}[x]$, and for each $n \geq 1$, factor $g\left(f^{n}(x)\right)$ in $\mathbb{F}_{q}[x]$ as

$$
g\left(f^{n}(x)\right)=p_{1}(x)^{e_{1}} p_{2}(x)^{e_{2}} \cdots p_{r}(x)^{e_{r}}
$$

with $p_{i}(x)$ irreducible in $\mathbb{F}_{q}[x]$.

(a) Describe the behavior, as $n \rightarrow \infty$, of quantities such as

$$
r, \quad \sum_{i=1}^{r} e_{i}, \quad \max _{1 \leq i \leq r} e_{i}, \quad \max _{1 \leq i \leq r} \operatorname{deg} p_{i}(x) .
$$

(b) Describe or predict the factorization of $g\left(f^{n+1}(x)\right)$ from a given factorization of $g\left(f^{n}(x)\right)$.

(c) Answer the analogous questions for the iterates of a rational function $f(x) \in$ $\mathbb{F}_{q}(x)$. Note that the exponents $e_{1}, \ldots, e_{r}$ may now be both positive and negative, and hence it may be more natural to study $\sum\left|e_{i}\right|$ instead of $\sum e_{i}$, and $\max \left|e_{i}\right|$ instead of $\max e_{i}$.

Under the assumptions that $q$ is odd, that $f(x)$ is quadratic, that $g(x)$ is irreducible of even degree, and that every iterate $f^{n}(x)$ is separable, Boston, Goksel, Jones, and Xia 98, 126] describe a conjectural multistage Markov model to explain the factorization of successive values of $g\left(f^{n}(x)\right)$. See also [100] for estimates for the number and degree of the irreducible factors of iterates of $f(x) \in \mathbb{F}_{q}[x]$, and see Section 19 for a further discussion of factorization of iterates over both finite fields and number fields. 
We might also look at orbits of dominant rational maps $f: \mathbb{P}^{N} \rightarrow \mathbb{P}^{N}$ over finite fields, although just as in Section 7 , we need to avoid points $P \in \mathbb{P}^{N}\left(\mathbb{F}_{q}\right)$ whose orbit lands in the indeterminacy locus $I(f)$ of $f$. We recall our general notation $\mathbb{P}_{f}^{N}(K)$ for the set of points $P \in \mathbb{P}^{N}(K)$ for which $f^{n}(P) \notin I(f)$ for all $n \geq 1$.

Conjecture 18.10. Let $f: \mathbb{P}^{N} \rightarrow \mathbb{P}^{N}$ be a dominant rational map defined over $\mathbb{F}_{q}$.

(a) $\mathbb{P}_{f}^{N}\left(\overline{\mathbb{F}}_{q}\right)$ is Zariski dense in $\mathbb{P}^{N}$.

(b) $\lim _{k \rightarrow \infty} \# \mathbb{P}_{f}^{N}\left(\mathbb{F}_{q^{k}}\right) / \# \mathbb{P}^{N}\left(\mathbb{F}_{q^{k}}\right)=1$.

Remark 18.11. We note that (b) implies (a) in Conjecture 18.10 Assuming that the conjecture is true, we may ask for a more accurate estimate for the ratio in (b). Thus let $m=\operatorname{codim} I(f)$. Then a rough probabilistic model suggests that for most (all?) maps $f$ and all $\epsilon>0$, there should be a constant $C(f, \epsilon)>0$ so that for all $k \geq 1$,

$$
1-C q^{-\left(m-\frac{1}{2}+\epsilon\right) k} \leq \# \mathbb{P}_{f}^{N}\left(\mathbb{F}_{q^{k}}\right) / \# \mathbb{P}^{N}\left(\mathbb{F}_{q^{k}}\right) \leq 1-C q^{-\left(m-\frac{1}{2}-\epsilon\right) k} .
$$

Remark 18.12. There is also the subject of permutation polynomials, which are polynomials $f(x) \in \mathbb{F}_{q}[x]$ having the property that the map $f: \mathbb{F}_{q} \rightarrow \mathbb{F}_{q}$ is a bijection. Since they act as permutations of $\mathbb{F}_{q}$, one might expect that their dynamics mirrors that of random automorphisms, rather than random endomorphisms. In any case, the study of permutation polynomials is a field in its own right, with many interesting open questions; see for example, [151,152] and [153, Chapter 7].

\section{IRREDUCIBILTy AND STABILITY OF ITERATES}

Let $K$ be a number field, and let $f(x) \in K(x)$ be a rational function of degree at least 2. Write the $n$th iterate of $f(x)$ as $f^{n}(x)=F_{n}(x) / G_{n}(x)$ with relatively prime polynomials $F_{n}(x), G_{n}(x) \in K[x]$. Then for $\alpha \in K$, we see that the polynomial

$$
F_{n}(x)-\alpha G_{n}(x) \text { is the numerator of the rational function } f^{n}(x)-\alpha .
$$

Definition 19.1. The pair $(f, \alpha)$ is said to be stable over $K$ if for every $n \geq 1$, the polynomial $F_{n}(x)-\alpha G_{n}(x)$ is irreducible in $K[x]$.

We observe that stability is not preserved under field extension 30 since for example $(f, \alpha)$ is not stable over the field $K\left(f^{-1}(\alpha)\right)$. This prompts the following more resilient version of stability, as described in [123, 127.

Definition 19.2. The pair $(f, \alpha)$ is eventually stable over $K$ if there is an $m \geq 0$ such that for every $\beta \in f^{-m}(\alpha)$, the pair $(f, \beta)$ is stable over $K(\beta)$. This is equivalent [127, Proposition 2.1(e)] to the existence of a bound $C(f, \alpha)$ such that the following holds:

For every $n \geq 1$, the polynomial $F_{n}(x)-\alpha G_{n}(x)$ is a product of at most $C(f, \alpha)$ irreducible factors in $K[x]$, counted with multiplicity.

\footnotetext{
${ }^{30}$ One might say that "stability is not very stable!" More seriously, there are two notions of stability in play, one being stability for varying $n$, the other for varying base field.
} 
Example 19.3. Let $f(x)=x^{2}+\frac{1}{48}$. Then initially the number of factors grows,

$$
\begin{aligned}
& f^{2}(x)=\left(x^{2}-\frac{1}{2} x+\frac{7}{48}\right)\left(x^{2}+\frac{1}{2} x+\frac{7}{48}\right) \\
& f^{3}(x)=\left(x^{2}-\frac{1}{2} x+\frac{19}{48}\right)\left(x^{2}+\frac{1}{2} x+\frac{19}{48}\right)\left(x^{4}-\frac{11}{24} x^{2}+\frac{313}{2304}\right) .
\end{aligned}
$$

Further, for all $n \geq 3$ one can show that $f^{n}(x)$ has exactly three irreducible factors in $\mathbb{Q}[x]$, so in (19.1) we can take $C\left(x^{2}+\frac{1}{48}, 0\right)=3$; see [55]. In particular, $\left(x^{2}+\frac{1}{48}, 0\right)$ is eventually stable over $\mathbb{Q}$.

Conjecture 19.4 (Jones-Levy, 2017, 127, Conjecture 1.2]). Let $K$ be a number field, let $f(x) \in K(x)$ be a rational function of degree at least 2 , and let $\alpha \in K$ be a point that is not periodic for $f$. Then $(f, \alpha)$ is eventually stable over $K$.

One might be bold and ask if there is a uniform bound for the eventual stability predicted by Conjecture 19.4 but some care is needed, since it is not hard to see that for any $k \geq 1$, the constant $C\left(f, f^{k}(\alpha)\right)$ in Definition 19.2 must be at least $k$.

Question 19.5. Let $K$ be a number field, let $f \in \operatorname{End}_{d}^{1}(K)$, and let $\alpha \in K$ have the property that $f^{-1}(\alpha) \cap \mathbb{P}^{1}(K)=\emptyset$. Is there a constant $\kappa(d, K)$ so that the statement (19.1) in Definition 19.2 is true with $C(f, \alpha)=\kappa(d, K)$ ? In particular, may we take $\kappa(2, \mathbb{Q})=3$ (cf. Example 19.3)? Might the same statement be true for a constant $\kappa$ depending only on $d$ and $[K: \mathbb{Q}]$ ?

Remark 19.6. Sookdeo 225] has shown that Conjecture19.4 for $\alpha$ wandering follows from the dynamical Lehmer conjecture (Conjecture 15.2).

Remark 19.7. We note that stability is closely related to the arboreal representation of the pair $(f, \alpha)$; cf. Section 5 . Thus $(f, \alpha)$ is stable over $K$ if and only if for every $n \geq 1$ we have

$$
\# f^{-n}(\alpha)=(\operatorname{deg} f)^{n} \text { and } \operatorname{Gal}(\bar{K} / K) \text { acts transitively on } f^{-n}(\alpha) \text {. }
$$

Eventual stability has a similar interpretation since it is defined in terms of stability of the pairs $(f, \beta)$ with $\beta \in f^{-m}(\alpha)$.

There are various equivalent ways to extend the notion of stability to finite endomorphisms of higher dimensional varieties such as $\mathbb{P}^{N}$. We give one such definition. Let $X \subsetneq \mathbb{P}^{N}$ be a proper subvariety of dimension $r$ that is defined over $K$, e.g., $X$ could be a point or a hypersurface. For any endomorphism $f \in \operatorname{End}_{d}^{N}(K)$, the pullback of $X$ has the form

$$
f^{*} X=\sum_{\substack{Y / K \subset \mathbb{P}^{N}, \operatorname{dim}(Y)=r \\ Y \text { irreducible over } K}} m(f, X ; Y) Y,
$$

i.e., $f^{*} X$ is a formal sum of $K$-irreducible $r$-dimensional subvarieties of $\mathbb{P}^{N}$ with multiplicities $m(f, X ; Y) \in \mathbb{Z}$.

Definition 19.8. Let $X \subsetneq \mathbb{P}^{N}$ be an $r$-dimensional subvariety defined over $K$, and let $f: \mathbb{P}^{N} \rightarrow \mathbb{P}^{N}$ be an endomorphism defined over $K$. The pair $(f, X)$ is eventually stable over $K$ if there is a constant $C(f, X)$ such that

$$
\sup _{\substack { n \geq 1 \\
\begin{subarray}{c}{Y / K \subset \mathbb{P}^{N}, \operatorname{dim}(Y)=r \\
Y \text { irreducible over } K{ n \geq 1 \\
\begin{subarray} { c } { Y / K \subset \mathbb { P } ^ { N } , \operatorname { d i m } ( Y ) = r \\
Y \text { irreducible over } K } }\end{subarray}} m\left(f^{n}, X ; Y\right) \leq C(f, X) .
$$


The pair $(f, X)$ is said to be geometrically eventually stable if it is eventually stable over $\bar{K}$, an algebraic closure of $K$. Geometric eventual stability essentially never happens if $\operatorname{dim}(X)=0$, but should be common for $\operatorname{dim}(X) \geq 1$.

Conjecture 19.9. Let $K$ be a number field, let $f: \mathbb{P}^{N} \rightarrow \mathbb{P}^{N}$ be an endomorphism of degree at least 2 defined over $K$, and let $P \in \mathbb{P}^{N}(K)$ be a point that is not periodic for $f$. Then $(f, P)$ is eventually stable over $K$.

Question 19.10. Let $K$ be a number field, let $f: \mathbb{P}^{N} \rightarrow \mathbb{P}^{N}$ be an endomorphism of degree at least 2 defined over $K$, and let $X \subsetneq \mathbb{P}^{N}$ be a subvariety that is defined over $K$ and irreducible over $K$.

(a) If the iterated forward orbit $\bigcup_{n \geq 1} f^{n}(X)$ of $X$ is Zariski dense in $\mathbb{P}^{N}$, is it true that $(f, X)$ is eventually stable over $K$ ?

(b) If the iterated forward orbit is Zariski dense and $\operatorname{dim}(X) \geq 1$, is it true that $(f, X)$ is geometrically eventually stable?

(c) If $\operatorname{dim}(X) \geq 1$, is eventual stability over $K$ equivalent to geometric eventual stability?

The question of (eventual) stability, or lack thereof, for maps over finite fields has also attracted attention. We restrict our attention to $\mathbb{P}^{1}$, and via an affine change of coordinates $f(x+\alpha)-\alpha$, we note that it suffices to discuss stability with base point 0 . An initial question is to determine the number of stable rational maps or polynomials.

Question 19.11. We recall that $\operatorname{End}_{d}^{1}(K)$ denotes the set of degree $d$ rational functions $f(x) \in K(x)$, and we write $\operatorname{Poly}_{d}^{1}(K)$ for the set of degree $d$ polynomials $f(x) \in K[x]$. We define

$$
\begin{aligned}
\operatorname{Poly}_{d}^{1}\left(\mathbb{F}_{q}\right)^{\text {stab }} & :=\left\{f \in \operatorname{Poly}_{d}^{1}\left(\mathbb{F}_{q}\right):(f, 0) \text { is stable over } \mathbb{F}_{q}\right\}, \\
\operatorname{Rat}_{d}^{1}\left(\mathbb{F}_{q}\right)^{\text {stab }} & :=\left\{f \in \operatorname{Rat}_{d}^{1}\left(\mathbb{F}_{q}\right):(f, 0) \text { is stable over } \mathbb{F}_{q}\right\},
\end{aligned}
$$

to be the indicated sets of $\mathbb{F}_{q}$-stable maps. For fixed $d \geq 2$, how does the size of the sets $\operatorname{Poly}_{d}^{1}\left(\mathbb{F}_{q}\right)^{\text {stab }}$ and $\operatorname{Rat}_{d}^{1}\left(\mathbb{F}_{q}\right)^{\text {stab }}$ grow as $q \rightarrow \infty$ ? Analogous questions apply for the sets of eventually stable maps, and for maps of $\mathbb{A}^{N}$ or $\mathbb{P}^{N}$ with $N \geq 2$.

Very little is known about Question 19.11, For polynomials of degree 2, Gomez and Nicolás 99 prove that there is an absolute constant $C$ so that

$$
\frac{1}{4}(q-1)^{2} \leq \# \operatorname{Poly}_{2}^{1}\left(\mathbb{F}_{q}\right)^{\text {stab }} \leq C q^{5 / 2} \log q .
$$

Note that the upper bound is nontrivial, since

$$
\operatorname{Poly}_{2}^{1}\left(\mathbb{F}_{q}\right)=\left\{a x^{2}+b x+c: a \in \mathbb{F}_{q}^{*}, b, c \in \mathbb{F}_{q}\right\}
$$

has roughly $q^{3}$ elements.

In a somewhat different vein, for a given finite set of polynomials $F \subset \mathbb{F}_{q}[x]$, let $\mathcal{C}(F)$ denote the set of all compositions of the elements of $F$, where each $f(x) \in F$ may be used arbitrarily many times. If $F$ is a set of monic quadratic polynomials, Ferraguti, Micheli, and Schnyder [78] give necessary and sufficient conditions for $\mathcal{C}(F)$ to consist entirely of irreducible polynomials, and they show [79] that the set of irreducible polynomials in $\mathcal{C}(F)$ has the structure of a regular language. Further, Heath-Brown and Micheli [108] prove that for every $k \geq 1$ there exists a set $F$ containing $k$ distinct quadratic polynomials such that $\mathcal{C}(F)$ consists entirely of irreducible polynomials. 
In those cases that $f(x)$ is not eventually stable, it is an interesting problem to describe the limiting distribution of the decomposition of $f^{n}(x)$ into irreducible factors. See Question 18.9 and the subsequent material in Section 18 for a brief discussion.

We might instead fix a map in $\mathbb{Q}(x)$, reduce it modulo $p$, and consider its stability over $\mathbb{F}_{p}$.

Question 19.12. Let $f(x) \in \mathbb{Q}(x)$ be a rational function of degree $d \geq 2$, and for each prime $p$, let $\tilde{f}_{p}(x) \in \mathbb{F}_{p}(x)$ denote its reduction modulo $p$; see Section 12 .

(a) For which $f(x)$ is it true that the set

$$
\left\{p: \tilde{f}_{p}(x) \text { is stable over } \mathbb{F}_{p}\right\}
$$

is finite?

(b) For which $f(x)$ is it true that the set

$$
\left\{p: \tilde{f}_{p}(x) \text { is eventually stable over } \mathbb{F}_{p}\right\}
$$

is finite?

As a specific example, Jones [124, Conjecture 6.3] has conjectured that $x^{2}+1$ is stable over $\mathbb{F}_{p}$ if and only if $p=3$, i.e., for $f(x)=x^{2}+1$, the set (19.3) is $\{3\}$. Ferraguti 77] has shown that under some Galois-theoretic assumptions, the set of stable primes (19.3) has density 0 . Working more generally over number fields, for most PCF maps the set (19.3) is infinite [124, Theoram 6.1], and indeed one might guess that the set (19.4) is infinite for all quadratic PCF maps.

\section{Primes, PRIME DIVISORS, AND PRIMITIVE DIVISORS IN ORBITS}

The study of primes appearing in sequences is a recurring theme in number theory, ranging from Dirichlet's theorem on primes in arithmetic progressions to the conjectural existence of infinitely many primes in the sequences $n^{2}+1$ and $2^{n}-1$. It is thus natural to look for primes appearing in orbits. A classical example is provided by the orbit of 3 under the map $f(x)=x^{2}-2 x+2$, which leads to the sequence of Fermat numbers

$$
f^{n}(3)=2^{2^{n}}+1
$$

In this case it is known that $f^{n}(3)$ is prime for $0 \leq n \leq 4$ and that $f^{n}(3)$ is composite for $5 \leq n \leq 32$.

Remark 20.1. We give a heuristic calculation to estimate the number of primes in an orbit. Let $f(x) \in \mathbb{Z}[x]$ have degree $d \geq 2$, and let $\alpha \in \mathbb{Z}$ be a wandering point for $f$. Then as described in Section 15, the canonical height $\hat{h}_{f}(\alpha)$ is strictly positive, which shows that $\log \left|f^{n}(\alpha)\right|=d^{n} \hat{h}_{f}(\alpha)+O(1)$ grows quite rapidly. If we assume that the integers $f^{n}(\alpha)$ in the orbit of $\alpha$ are random and independent in an appropriate sense, then the prime number theorem says that the probability 
that $\left|f^{n}(\alpha)\right|$ is prime is roughly $1 / \log \left|f^{n}(\alpha)\right|$. This leads to the estimate

$$
\begin{aligned}
\#\left\{n \geq 0:\left|f^{n}(\alpha)\right| \text { is prime }\right\} & \approx \sum_{n \geq 0} \operatorname{Prob}\left(\left|f^{n}(\alpha)\right| \text { is prime }\right) \\
& \approx \sum_{n \geq 0} \frac{1}{\log \left|f^{n}(\alpha)\right|} \\
& \approx \sum_{n \geq 0} \frac{1}{d^{n} \hat{h}_{f}(\alpha)+O(1)}<\infty .
\end{aligned}
$$

The convergence of this series suggests that $\mathcal{O}_{f}(\alpha)$ should contain only finitely many primes. However - and this is a major caveat - our random and independent assumption has some definite flaws. For example, the Fermat numbers (20.1) are pairwise relatively prime, and their prime divisors have congruence constraints.

Conjecture 20.2. Let $f(x) \in \mathbb{Z}[x]$ be a polynomial of degree at least 2 , and let $\alpha \in$ $\mathbb{Z}$ be a wandering point for $f$. Then $\mathcal{O}_{f}(\alpha)$ contains only finitely many primes.

More generally, let $K$ be a number field. For each prime ideal $\mathfrak{p}$ of the ring of integers of $K$, let $\operatorname{ord}_{\mathfrak{p}}: K^{*} \rightarrow \mathbb{Z}$ be the associated normalized valuation, and let $\operatorname{ord}_{\mathfrak{p}}^{+}(\alpha)=\max \left\{\operatorname{ord}_{\mathfrak{p}}(\alpha), 0\right\}$. Then we have the following natural generalization of Conjecture 20.2 .

Conjecture 20.3. Let $K$ be a number field, let $f(x) \in K(x)$ be a rational function of degree at least 2 , let $\alpha \in K$ be a wandering point for $f$, let $\beta \in K$, and let $c$ be a constant. Then the set

$$
\left\{n \geq 0: \sum_{\mathfrak{p}} \operatorname{ord}_{\mathfrak{p}}^{+}\left(f^{n}(\alpha)-\beta\right) \leq c\right\}
$$

is finite.

Conjectures 20.2 and 20.3 suggest that orbits contain very few primes, but even for sequences such as $n^{2}+1$ and $2^{n}-1$, the existence of infinitely many primes is still conjectural. However, there are many situations, ranging from logic to group theory to dynamics, where it suffices to know that most terms in the sequence are divisible by a new prime not dividing any previous term. These considerations prompt the following definitions.

Definition 20.4. Let $\mathcal{A}=\left(a_{1}, a_{2}, a_{3}, \ldots\right)$ be a sequence of integers. The suppor 31 of $\mathcal{A}$ is the set of primes

$$
\operatorname{Support}(\mathcal{A}):=\left\{\text { primes } p: p \text { divides at least one nonzero } a_{n}\right\} \text {. }
$$

Definition 20.5. A prime $p$ is said to be a primitive prime divisor of $a_{n}$ if $p$ divides $a_{n}$ and $p$ does not divide $a_{k}$ for all $k<n$ with $a_{k} \neq 0$. The Zsigmondy set of $\mathcal{A}$ is the set of indices whose terms do not have a primitive prime divisor,

$$
\operatorname{Zsig}(\mathcal{A}):=\left\{n: a_{n} \neq 0 \text { and } a_{n} \text { has no primitive prime divisors }\right\} .
$$

More generally, if $\mathcal{A}$ is a sequence of rational numbers, then $\operatorname{Support}(\mathcal{A})$ and $\operatorname{Zsig}(\mathcal{A})$ are defined, respectively, to be the support and the Zsigmondy set of the sequence of numerators of $a_{1}, a_{2}, \ldots$.

\footnotetext{
${ }^{31}$ The fancy reason for the name "support" is that a nonzero integer a may be viewed as the function on $\operatorname{Spec}(\mathbb{Z})$ that sends the prime ideal $(p) \in \operatorname{Spec}(\mathbb{Z})$ to the value $\bar{a} \in \mathbb{Z} / p \mathbb{Z}$. The support of $a$ is then the set of points of $\operatorname{Spec}(\mathbb{Z})$ at which $a$ vanishes.
} 
Example 20.6. Let $\alpha \in \mathbb{Q}$ be a rational number with $\alpha \notin\{0, \pm 1\}$. In 1892 Zsigmondy [249], generalizing an earlier weaker result of Bang, proved that

$$
\text { Zsig }\left(\left(\alpha^{n}-1\right)_{n \geq 1}\right) \subseteq\{1,2,3,6\} .
$$

Carmichael 49 proved in 1913 that the Zsigmondy set of the Fibonacci sequence is $\{1,2,6,12\}$, and recently, Bilu, Hanrot, and Voutier [38 generalized all of these old results by showing that if $\mathcal{A}$ is a Lucas sequence or a Lehmer sequence, then $\operatorname{Zsig}(\mathcal{A}) \subseteq\{1,2, \ldots, 30\}$.

Let $f(x) \in \mathbb{Q}(x)$ be a rational function of degree at least 2 , and let $\alpha \in \mathbb{Q}$ be an $f$-wandering point. Then for any $\beta \in \mathbb{Q}$, we would like to understand the support and the Zsigmondy set of the dynamically defined sequence

$$
\mathcal{A}(f, \alpha, \beta):=\left(f^{n}(\alpha)-\beta\right)_{n \geq 1} .
$$

Theorem 20.7 (Ingram-Silverman, 2009, [122). Let $f(x) \in \mathbb{Q}(x)$ be a rational function of degree at least 2 , let $\alpha \in \mathbb{Q}$ be an $f$-wandering point, and let $\beta \in \mathbb{Q}$ be an $f$-preperiodic point. Assume further that for all $k \geq 1$, the equation $f^{k}(x)=\beta$ has at least one complex root other than $x=\beta$. Then the Zsigmondy set of $\mathcal{A}(f, \alpha, \beta)$ is finite.

If the point $\beta$ in Theorem 20.7 is allowed to be an $f$-wandering point, then the problem becomes much more difficult. In 2013 Krieger [139] has shown that for all $d \geq 2$ and all $c \in \mathbb{Q}$ with $c \notin\{0,-1,-2\}$,

$$
\# \operatorname{Zsig}\left(\mathcal{A}\left(x^{d}+c, 0,0\right)\right) \leq 23 .
$$

Notice that the point 0 is a critical wandering point of $x^{d}+c$. There are stronger conditional results if one assumes the $A B C$-conjecture [102, 158, or Vojta's conjecture 219. See also 64 for a proof that the Zsigmondy set of a sequence of the form $\left(f^{n}(\alpha)-f^{n-1}(\alpha)\right)_{n \geq 1}$ is finite.

Conjecture 20.8. Let $f(x) \in \mathbb{Q}(x)$ be a rational function of degree at least 2 , and let $\alpha, \beta \in \mathbb{Q}$ be $f$-wandering points. Then the Zsigmondy set of $\mathcal{A}(f, \alpha, \beta)$ is finite.

Conjecture 20.8 implies, in particular, that there are infinitely many primes dividing at least one term in the sequence $\mathcal{A}(f, \alpha, \beta)$. Could it happen that every prime divides at least one term? Or is the set of such primes relatively sparse?

There is a large literature studying the density of the support of sequences such as $a^{n}-1$ and the Fibonacci sequence. In the dynamical setting, it is interesting to study the density of the support of orbits, as in the next theorem, which generalizes an earlier result of Odoni [183] for $f(x)=x^{2}-x+1$. (We refer the reader to Definition 3.1 for the definition of the density of a set of primes.)

Theorem 20.9 (Jones, 2008, 123). Let $\alpha \in \mathbb{Z}$. Then

$$
\begin{aligned}
\operatorname{Density}\left(\operatorname{Support}\left(\mathcal{A}\left(x^{2}+c, \alpha, 0\right)\right)\right) & =0 & & \text { for all } c \in \mathbb{Z} \backslash\{-1\}, \\
\operatorname{Density}\left(\operatorname{Support}\left(\mathcal{A}\left(x^{2}-c x+c, \alpha, 0\right)\right)\right) & =0 & & \text { for all } c \in \mathbb{Z} .
\end{aligned}
$$

See 104 for a generalization of Theorem 20.9 to polynomials of the form $x^{d}+c$ over number fields and function fields. But before one guesses that dynamical densities are always zero, we note $([101,123])$

$$
\begin{aligned}
\operatorname{Density}\left(\operatorname{Support}\left(\mathcal{A}\left(x^{2}, q, q\right)\right)\right) & =1 / 3 \text { for all primes } q \geq 3, \\
\operatorname{Density}\left(\operatorname{Support}\left(\mathcal{A}\left(x^{2}-2,16,16\right)\right)\right) & =7 / 24 .
\end{aligned}
$$


As the following conjecture shows, it is no coincidence that these examples use $x^{2}$ and $x^{2}-2$, since they are PCF polynomials.

Conjecture 20.10 (Jones, 2008, [123, Conjecture 4.7]). Let $f(x) \in \mathbb{Z}[x]$ be a monic polynomial of degree 2 , and assume that $f(x)$ is stable 32 and that its critical point is wandering. Then

$$
\operatorname{Density}(\operatorname{Support}(\mathcal{A}(f, \alpha, 0)))=0 \quad \text { for all } \alpha \in \mathbb{Z} \text {. }
$$

Question 20.11. Give a concise characterization, analogous to that given in Conjecture 20.10 for maps satisfying $\operatorname{deg}(f)=2$, which ensures that $f(x) \in \mathbb{Q}(x)$ and $\alpha, \beta \in \mathbb{Q}$ satisfy

$$
\operatorname{Density}(\operatorname{Support}(\mathcal{A}(f, \alpha, \beta)))=0 \text {. }
$$

\section{INTEGRAL POINTS IN ORBITS}

Let $f(x) \in \mathbb{Q}(x)$ be a rational function of one variable, and let $\alpha \in \mathbb{Q}$ be a rational starting point. A natural number theoretic question is to ask whether the orbit $\mathcal{O}_{f}(\alpha)$ contains infinitely many integers. In some cases, for example $f(x) \in \mathbb{Z}[x]$ and $\alpha \in \mathbb{Z}$, the entire orbit consists of integers. Similarly, for the map $f(x)=1 / x^{2}$ and $\alpha \geq 2$ an integer, every other entry in the orbit is an integer, which simply reflects the fact that $f^{2}(x)=x^{4}$ is a polynomial. The following proposition is well-known to dynamicists. For a generalization to endomorphisms of $\mathbb{P}^{N}$, see [83, Proposition 4.2] and [214, Exercise 7.16], and for a characteristic $p$ version, see [212.

Proposition 21.1. Let $f(x) \in \mathbb{C}(x)$ be a rational function of degree $d \geq 2$ such that there is an $n \geq 1$ with $f^{n}(x) \in \mathbb{C}[x]$. Then already $f^{2}(x) \in \mathbb{C}[x]$.

We start with an arithmetic analogue of Proposition 21.1 which is at the same time a dynamical analogue of Siegel's theorem that curves of genus at least 1 have only finitely many integral points.

Theorem 21.2 (Silverman, 1993, [210]). Let $f(x) \in \mathbb{Q}(x)$ be a rational function of degree at least 2 , and assume that $f^{2}(x)$ is not a polynomial. Then for all $\alpha \in \mathbb{Q}$, the orbit $\mathcal{O}_{f}(\alpha)$ contains only finitely many integers.

A strong version of Siegel's theorem, alluded to earlier, puts a limit on the allowable nonintegrality of points on curves. Here is a dynamical analogue.

Theorem 21.3 (Dynamical Siegel Theorem for $\mathbb{P}^{1}$ : Silverman, 1993, 210]). Let $f(x) \in \mathbb{Q}(x)$ be a rational function of degree at least 2 , and assume that both $f^{2}(x)$ and $f^{2}\left(x^{-1}\right)^{-1}$ are not polynomials. Let $\alpha \in \mathbb{Q}$ be a wandering point for $f$, and write $f^{n}(\alpha)$ as a fraction

$$
f^{n}(\alpha)=A_{n} / B_{n} \quad \text { with } A_{n}, B_{n} \in \mathbb{Z} \text { and } \operatorname{gcd}\left(A_{n}, B_{n}\right)=1 .
$$

Then

$$
\lim _{n \rightarrow \infty} \frac{\log \left|A_{n}\right|}{\log \left|B_{n}\right|}=1
$$

\footnotetext{
${ }^{32}$ We recall from Section 19 that $f(x)$ is stable if every iterate $f^{n}(x)$ is irreducible in $\mathbb{Q}[x]$.
} 
In words, Theorem 21.3 says that the numerator and the denominator of $f^{n}(\alpha)$ have roughly the same number of digits when $n$ is large.

Lang formulated a conjectural generalization of Siegel's theorem to integral points on affine subvarieties of abelian varieties, and Faltings 68, 69. proved Lang's conjecture. We give a conjectural dynamical analogue for $\mathbb{P}^{N}$. We say that a point $P \in \mathbb{P}^{N}(\mathbb{Q})$ is an integral point (relative to the hyperplane $x_{0}=0$ ) if it has homogeneous coordinates of the form

$$
P=\left[1, a_{1}, a_{2}, \ldots, a_{N}\right] \quad \text { with } a_{1}, \ldots, a_{N} \in \mathbb{Z} .
$$

Conjecture 21.4 (Dynamical Lang-Siegel Conjecture for $\mathbb{P}^{N}$ ). Let $f: \mathbb{P}^{N} \rightarrow \mathbb{P}^{N}$ be a morphism of degree $d \geq 2$ defined over $\mathbb{Q}$, and assume that for all $1 \leq n \leq$ $N+1$, the first coordinate function of $f^{n}$ is not a constant multiple of $X_{0}^{d^{n}}$. Let $P \in \mathbb{P}^{N}(\mathbb{Q})$. Then $\mathcal{O}_{f}(P)$ contains only finitely many points that are integeral relative to the hyperplane $x_{0}=0$. More precisely, assume that $P$ is wandering, and for each $n \geq 1$, write

$$
\begin{aligned}
f^{n}(P)=\left[A_{0}(n),\right. & \left.A_{1}(n), \ldots, A_{N}(n)\right] \\
& \text { with } A_{0}(n), \ldots, A_{N}(n) \in \mathbb{Z} \text { and } \operatorname{gcd}\left(A_{0}(n), \ldots, A_{N}(n)\right)=1 .
\end{aligned}
$$

Then

$$
\lim _{n \rightarrow \infty} \frac{\log \left|A_{0}(n)\right|}{\log \max \left\{\left|A_{0}(n)\right|, \ldots,\left|A_{N}(n)\right|\right\}}=1 .
$$

Remark 21.5. All of the theorems/conjectures in this section can be proven/stated for number fields $K$ and for points that are integral in rings of $S$-integers of $K$, but we have restricted our attention to $\mathbb{Q}$ in order to keep the notation and the technicalities to a minimum.

\section{LOCAL-GLOBAL QUESTIONS IN DYNAMICS}

An enduring theme in mathematics is the use of local data to characterize global phenomena. In number theory, local data can mean information modulo $m$ for all, or at least most, integers $m$. The Chinese remainder theorem reduces studying solutions modulo $m$ to studying solutions modulo prime powers, and the latter are best described in terms of solutions in the field of $p$-adic numbers $\mathbb{Q}_{p}$.

For example, a classical theorem of Legendre says that for $A, B, C \in \mathbb{Z}$, the equation

$$
A X^{2}+B Y^{2}+C Z^{2}=0
$$

has a nontrivial solution in integers if and only if it has a nontrivial solution in $\mathbb{Q}_{p}$ for every prime $p$ and a nontrivial solution in $\mathbb{R}$. A modern example of a localglobal principle is the conjecture of Birch and Swinnerton-Dyer, which describes the $\mathbb{Q}$-rational points on an elliptic curve in terms of its $\mathbb{R}$ points and its $\mathbb{Q}_{p}$ points as $p$ ranges over all primes.

There are various natural ways in which one might formulate local-global problems in arithmetic dynamics. For example, to what extent does the existence of $\mathbb{Q}_{p}$ and $\mathbb{R}$ periodic points force there to be $\mathbb{Q}$ periodic points? The following result, although covering only a few cases, gives a flavor for what we have in mind. (See also 12,13 .) 
Theorem 22.1 (Krumm, 2016, 140]). Let $f(x)=a x^{2}+b x+c \in \mathbb{Q}[x]$ be a quadratic polynomial. Let $n \in\{1,2,3\}$. Suppose that for every prime $p$, the polynomial $f(x)$ has a $\mathbb{Q}_{p}$-rational periodic point of exact period $n$. Then $f(x)$ has a $\mathbb{Q}$-rational periodic point of exact period $n$. Further, for $n=4$ and, under an additional assumption, for $n=5$, there exist infinitely many primes $p$ such that $f(x)$ does not have a $\mathbb{Q}_{p}$-rational periodic point of exact period $n$.

This may be compared with Theorem 4.4

We formulate the next question over $\mathbb{Q}$, but it is easily generalized to number fields.

Question 22.2. Let $f: \mathbb{P}^{N} \rightarrow \mathbb{P}^{N}$ be a morphism of degree $d$ defined over $\mathbb{Q}$.

(a) For which $n \geq 1$ is it true that $f$ has a point of exact period $n$ in $\mathbb{P}^{N}(\mathbb{Q})$ if and only if $f$ has a point of exact period $n$ in $\mathbb{P}^{N}(\mathbb{R})$ and in $\mathbb{P}^{N}\left(\mathbb{Q}_{p}\right)$ for all primes $p$ ?

(b) For which $n \geq 1$ is it true that there are infinitely many primes $p$ such that the map $f$ has no points of exact period $n$ in $\mathbb{P}^{N}\left(\mathbb{Q}_{p}\right)$ ? Is this true for all $n \geq n_{0}(N, d)$ ?

More generally, we may ask the same questions for preperiodic points of type $(m, n)$, i.e., points with tails of exact length $m$ and periods of exact length $n$.

One can also formulate local-global questions related to the Dynamical MordellLang Conjecture (Conjecture 7.1). We state the next conjecture using the topology of the adele ring of a number field $K$. This ring amalgamates all of the archimedean and $p$-adic data of $K$. The conjecture says, roughly, that $p$-adic data associated to the orbit of a point suffices to pin down the orbit.

Conjecture 22.3 (Dynamical Brauer-Manin Conjecture: Hsia-Silverman, 2009, [114]). Let $K$ be a number field, let $X / K$ be a variety, let $f: X \rightarrow X$ be a morphism defined over $K$, let $Y \subset X$ be a subvariety defined over $K$, and let $P \in X(K)$. Let $\mathcal{A}_{K}$ be the ring of adeles of $K$, and for any subset $S$ of $X(K)$, let $\mathcal{C}(S)$ denote the adelic closure of $S$ in $X\left(\mathcal{A}_{K}\right)$. Then

$$
\mathcal{C}\left(Y \cap \mathcal{O}_{f}(P)\right)=Y\left(\mathcal{A}_{K}\right) \cap \mathcal{C}\left(\mathcal{O}_{f}(P)\right) .
$$

Classically, a Diophantine equation

$$
F\left(x_{1}, \ldots, x_{n}\right)=0, \quad \text { where } F \in \mathbb{Z}\left[x_{1}, \ldots, x_{n}\right],
$$

is said to have a Brauer obstruction to $\mathbb{Q}$-rational solutions if either it has no solutions in $\mathbb{R}$ or if there exists a prime $p$ such that the equation has no solutions in $\mathbb{Q}_{p}$. When there is no Brauer obstruction, Manin devised a fancier test for the nonexistence of solutions. This has led to a considerable amount of research studying for which equations the lack of a Brauer-Manin obstruction suffices to ensure the existence of a $\mathbb{Q}$-rational solution. Conjecture 22.3 is a dynamical analogue of Scharaschkin's 202] geometric formulation of the Brauer-Manin obstruction for curves. Conjecture 22.3 is known for $X=\mathbb{P}^{1}$ and for various other special cases; see [4,224].

Here is another local-global formulation of dynamical Mordell-Lang conjecture. We state it for polynomials, but it would be interesting to formulate it for rational functions, or even for morphisms on $\mathbb{P}^{N}$.

Question 22.4. Let $K$ be a number field, let $f(x), g(x) \in K[x]$ be polynomials of degree at least 2 , and let $a, b \in K$. 
(a) Suppose that for all but finitely many primes $\mathfrak{p}$, the mod $\mathfrak{p}$ orbits of $a$ and $b$ satisfy

$$
\mathcal{O}_{\tilde{f}}(\tilde{a} \bmod \mathfrak{p}) \cap \mathcal{O}_{\tilde{g}}(\tilde{b} \bmod \mathfrak{p}) \neq \emptyset
$$

Is it true that true that $f$ and $g$ have the same complex Julia sets? 33 If the answer is affirmative, does it suffice to assume that (22.1) holds for a set of primes of density 1 ?

(b) Fix some $\epsilon>0$, and suppose that the intersection (22.1) has at least $\mathrm{Np}^{\epsilon}$ elements for infinitely many primes $\mathfrak{p}$. Is it true that true that $f$ and $g$ have the same complex Julia sets?

\section{ACKNOWLEDGMENTS}

The authors would like to thank Bryna Kra and Steven J. Miller for suggesting that this article be written, and Eric Bedford, Laura DeMarco, Mattias Jonsson, Juan Rivera-Letelier, Karou Sano, and Igor Shparlinski for helpful advice. They would also like to express their appreciation to the referee for their extensive list of comments and corrections.

\section{ABOUt THE AUTHORS}

Robert Benedetto is professor of mathematics at Amherst College. His research interests include arithmetic dynamics with a focus on non-archimedean dynamics.

Patrick Ingram is assistant professor at York University. His research interests include arithmetic dynamics, arithmetic geometry, and diophantine approximation.

Rafe Jones is associate professor of mathematics at Carleton College. His research interests include arithmetic dynamics with a focus on Galois-theoretic aspects.

Michelle Manes is professor of mathematics at the University of Hawaii at Manoa and a program officer at the National Science Foundation. Her primary research interests are in arithmetic geometry with a focus on arithmetic dynamics.

Joseph H. Silverman is professor of mathematics at Brown University. He is a recipient of an AMS Steele Prize and is a fellow of the AMS. His research interests include arithmetic geometry, arithmetic dynamics, and cryptography.

Thomas Tucker is professor of mathematics at University of Rochester. His research interests include arithmetic dynamics and diophantine geometry.

\section{REFERENCES}

[1] Amir Akbary and Dragos Ghioca, Periods of orbits modulo primes, J. Number Theory 129 (2009), no. 11, 2831-2842, DOI 10.1016/j.jnt.2009.03.007. MR2549537

[2] E. Amerik, F. Bogomolov, and M. Rovinsky, Remarks on endomorphisms and rational points, Compos. Math. 147 (2011), no. 6, 1819-1842, DOI 10.1112/S0010437X11005537. MR.2862064

[3] Ekaterina Amerik, Existence of non-preperiodic algebraic points for a rational self-map of infinite order, Math. Res. Lett. 18 (2011), no. 2, 251-256, DOI 10.4310/MRL.2011.v18.n2.a5. MR2784670

[4] Ekaterina Amerik, Pär Kurlberg, Khoa D. Nguyen, Adam Towsley, Bianca Viray, and José Felipe Voloch, Evidence for the dynamical Brauer-Manin criterion, Exp. Math. 25 (2016), no. 1, 54-65, DOI 10.1080/10586458.2015.1056889. MR3424832

\footnotetext{
${ }^{33}$ Aside from power maps and Chebyshev polynomials, having the same Julia set is more or less equivalent to $f$ and $g$ having a common iterate, up to symmetries of the Julia set.
} 
[5] V. I. Arnol'd, Dynamics of complexity of intersections, Bol. Soc. Brasil. Mat. (N.S.) 21 (1990), no. 1, 1-10, DOI 10.1007/BF01236277. MR 1139553

[6] D. K. Arrowsmith and F. Vivaldi, Some p-adic representations of the Smale horseshoe, Phys. Lett. A 176 (1993), no. 5, 292-294, DOI 10.1016/0375-9601(93)90920-U. MR.1218804

[7] David K. Arrowsmith and Franco Vivaldi, Geometry of p-adic Siegel discs, Phys. D 71 (1994), no. 1-2, 222-236, DOI 10.1016/0167-2789(94)90191-0. MR1264116

[8] Matthew Baker and Laura De Marco, Special curves and postcritically finite polynomials, Forum Math. Pi 1 (2013), e3, 35, DOI 10.1017/fmp.2013.2. MR3141413

[9] Matthew Baker and Laura DeMarco, Preperiodic points and unlikely intersections, Duke Math. J. 159 (2011), no. 1, 1-29, DOI 10.1215/00127094-1384773. MR2817647

[10] Matthew H. Baker and Robert Rumely, Equidistribution of small points, rational dynamics, and potential theory (English, with English and French summaries), Ann. Inst. Fourier (Grenoble) 56 (2006), no. 3, 625-688. MR2244226

[11] Matthew Baker and Robert Rumely, Potential theory and dynamics on the Berkovich projective line, Mathematical Surveys and Monographs, vol. 159, American Mathematical Society, Providence, RI, 2010. MR2599526

[12] Stefan Barańczuk, On a dynamical local-global principle in Mordell-Weil type groups, Expo. Math. 35 (2017), no. 2, 206-211, DOI 10.1016/j.exmath.2016.07.001. MR3654074

[13] Boris Bartolome, Yuri Bilu, and Florian Luca, On the exponential local-global principle, Acta Arith. 159 (2013), no. 2, 101-111, DOI 10.4064/aa159-2-1. MR.3062909

[14] Eric Bedford and Kyounghee Kim, Linear recurrences in the degree sequences of monomial mappings, Ergodic Theory Dynam. Systems 28 (2008), no. 5, 1369-1375, DOI 10.1017/S0143385708000242. MR2449533

[15] J. P. Bell, D. Ghioca, and T. J. Tucker, The dynamical Mordell-Lang problem for étale maps, Amer. J. Math. 132 (2010), no. 6, 1655-1675. MR2766180

[16] J. P. Bell, D. Ghioca, and T. J. Tucker, Applications of p-adic analysis to Burnside's problem and Zhang's conjecture, arXiv:1310.5775 (2013).

[17] Jason P. Bell, A generalised Skolem-Mahler-Lech theorem for affine varieties, J. London Math. Soc. (2) 73 (2006), no. 2, 367-379, DOI 10.1112/S002461070602268X. MR2225492

[18] Jason P. Bell, Dragos Ghioca, and Thomas J. Tucker, The dynamical Mordell-Lang conjecture, Mathematical Surveys and Monographs, vol. 210, American Mathematical Society, Providence, RI, 2016. MR3468757

[19] Elisa Bellah, Derek Garton, Erin Tannenbaum, and Noah Walton, A probabilistic heuristic for counting components of functional graphs of polynomials over finite fields, Involve 11 (2018), no. 1, 169-179, DOI 10.2140/involve.2018.11.169. MR.3681355

[20] M. P. Bellon and C.-M. Viallet, Algebraic entropy, Comm. Math. Phys. 204 (1999), no. 2, 425-437, DOI 10.1007/s002200050652. MR1704282

[21] Shahar Ben-Menahem, p-adic iterations, TAUP 1627-88, Tel-Aviv University, preprint (1988).

[22] Robert Benedetto, Patrick Ingram, Rafe Jones, and Alon Levy, Attracting cycles in p-adic dynamics and height bounds for postcritically finite maps, Duke Math. J. 163 (2014), no. 13, 2325-2356, DOI 10.1215/00127094-2804674. MR.3265554

[23] Robert Benedetto and Jamie Juul, Odoni's conjecture for number fields, arXiv:1803.01987 (2018).

[24] Robert L. Benedetto, Fatou components inp-adic dynamics, ProQuest LLC, Ann Arbor, MI, 1998. Thesis (Ph.D.)-Brown University. MR2697379

[25] Robert L. Benedetto, $p$-adic dynamics and Sullivan's no wandering domains theorem, Compositio Math. 122 (2000), no. 3, 281-298, DOI 10.1023/A:1002067315057. MR1781331

[26] Robert L. Benedetto, Hyperbolic maps in p-adic dynamics, Ergodic Theory Dynam. Systems 21 (2001), no. 1, 1-11, DOI 10.1017/S0143385701001043. MR 1826658

[27] Robert L. Benedetto, Reduction, dynamics, and Julia sets of rational functions, J. Number Theory 86 (2001), no. 2, 175-195, DOI 10.1006/jnth.2000.2577. MR.1813109

[28] Robert L. Benedetto, Examples of wandering domains in p-adic polynomial dynamics (English, with English and French summaries), C. R. Math. Acad. Sci. Paris 335 (2002), no. 7, 615-620. MR.1941304

[29] Robert L. Benedetto, Wandering domains and nontrivial reduction in non-Archimedean dynamics, Illinois J. Math. 49 (2005), no. 1, 167-193. MR2157374 
[30] Robert L. Benedetto, Wandering domains in non-Archimedean polynomial dynamics, Bull. London Math. Soc. 38 (2006), no. 6, 937-950, DOI 10.1112/S0024609306019126. MR2285248

[31] Robert L. Benedetto, Preperiodic points of polynomials over global fields, J. Reine Angew. Math. 608 (2007), 123-153, DOI 10.1515/CRELLE.2007.055. MR2339471

[32] Robert L. Benedetto, A criterion for potentially good reduction in non-archimedean dynamics, Acta Arith. 165 (2014), no. 3, 251-256, DOI 10.4064/aa165-3-4. MR3263950

[33] Robert L. Benedetto, Dynamics in one non-archimedean variable, Graduate Studies in Mathematics, vol. 198, American Mathematical Society, Providence, RI, 2019.

[34] Robert L. Benedetto, Ruqian Chen, Trevor Hyde, Yordanka Kovacheva, and Colin White, Small dynamical heights for quadratic polynomials and rational functions, Exp. Math. 23 (2014), no. 4, 433-447, DOI 10.1080/10586458.2014.938203. MR.3277939

[35] Robert L. Benedetto, Benjamin Dickman, Sasha Joseph, Benjamin Krause, Daniel Rubin, and Xinwen Zhou, Computing points of small height for cubic polynomials, Involve 2 (2009), no. 1, 37-64, DOI 10.2140/involve.2009.2.37. MR2501344

[36] Robert L. Benedetto, Dragos Ghioca, Pär Kurlberg, and Thomas J. Tucker, A case of the dynamical Mordell-Lang conjecture, Math. Ann. 352 (2012), no. 1, 1-26, DOI 10.1007/s00208010-0621-4. With an appendix by Umberto Zannier. MR 2885573

[37] Jean-Paul Bézivin, Sur les points périodiques des applications rationnelles en dynamique ultramétrique (French), Acta Arith. 100 (2001), no. 1, 63-74, DOI 10.4064/aa100-1-5. MR 1864626

[38] Yu. Bilu, G. Hanrot, and P. M. Voutier, Existence of primitive divisors of Lucas and Lehmer numbers, J. Reine Angew. Math. 539 (2001), 75-122, DOI 10.1515/crll.2001.080. With an appendix by M. Mignotte. MR 1863855

[39] Jérémy Blanc, Jung Kyu Canci, and Noam D. Elkies, Moduli spaces of quadratic rational maps with a marked periodic point of small order, Int. Math. Res. Not. IMRN 23 (2015), 12459-12489, DOI 10.1093/imrn/rnv063. MR3431627

[40] Sébastien Boucksom, Charles Favre, and Mattias Jonsson, Degree growth of meromorphic surface maps, Duke Math. J. 141 (2008), no. 3, 519-538, DOI 10.1215/00127094-2007-004. MR:2387430

[41] T. Bousch, Sur quelques problèmes de dynamique holomorphe. PhD thesis, Université de Paris-Sud, Centre d'Orsay, 1992.

[42] Nils Bruin and Alexander Molnar, Minimal models for rational functions in a dynamical setting, LMS J. Comput. Math. 15 (2012), 400-417, DOI 10.1112/S1461157012001131. MR 3015733

[43] Xavier Buff, On postcritically finite unicritical polynomials, https://www.math.univtoulouse.fr/ buff/Preprints/Gleason/Gleason.pdf (2017).

[44] Xavier Buff, A.L. Epstein, and Sarah Koch, Irreducibility and postcritically finite unicritical polynomials, arXiv.1806.11221 (2018).

[45] Jordan Cahn, Rafe Jones, and Jacob Spear, Powers in orbits of rational functions: cases of an arithmetic dynamical Mordell-Lang conjecture, arXiv:1512.03085 (2015).

[46] Gregory S. Call and Joseph H. Silverman, Canonical heights on varieties with morphisms, Compositio Math. 89 (1993), no. 2, 163-205. MR.1255693

[47] Jung Kyu Canci and Laura Paladino, Preperiodic points for rational functions defined over a global field in terms of good reduction, Proc. Amer. Math. Soc. 144 (2016), no. 12, 51415158, DOI 10.1090/proc/13096. MR3556260

[48] Serge Cantat and Junyi Xie, On degrees of birational mappings, arXiv:1802.08470 (2018).

[49] R. D. Carmichael, On the numerical factors of the arithmetic forms $\alpha^{n} \pm \beta^{n}$, Ann. of Math. (2) 15 (1913/14), no. 1-4, 30-48, DOI 10.2307/1967797. MR1502458

[50] Antoine Chambert-Loir, Mesures et équidistribution sur les espaces de Berkovich (French), J. Reine Angew. Math. 595 (2006), 215-235, DOI 10.1515/CRELLE.2006.049. MR2244803

[51] Mei-Chu Chang, Carlos D'Andrea, Alina Ostafe, Igor E. Shparlinski, and Martín Sombra, Orbits of polynomial dynamical systems modulo primes, Proc. Amer. Math. Soc. 146 (2018), no. 5, 2015-2025, DOI 10.1090/proc/13904. MR.3767353

[52] João Alberto de Faria and Benjamin Hutz, Automorphism groups and invariant theory on $\mathbb{P}^{N}$, J. Algebra Appl. 17 (2018), no. 9, 1850162, 38, DOI 10.1142/S0219498818501621. $\operatorname{MR} 3846410$ 
[53] Laura DeMarco, Bifurcations, intersections, and heights, Algebra Number Theory 10 (2016), no. 5, 1031-1056, DOI 10.2140/ant.2016.10.1031. MR3531361

[54] Laura DeMarco and Dragos Ghioca, Rationality of dynamical canonical height (to appear in Ergodic Theory Dynam. Systems), arXiv:1602.05614 (2018).

[55] David DeMark, Wade Hindes, Rafe Jones, Moses Misplon, and Michael Stoneman, Eventually stable quadratic polynomials over $\mathbb{Q}$, arXiv:1902.09220 (2019).

[56] Laurent Denis, Géométrie et suites récurrentes (French, with English and French summaries), Bull. Soc. Math. France 122 (1994), no. 1, 13-27. MR.1259107

[57] J. Diller and C. Favre, Dynamics of bimeromorphic maps of surfaces, Amer. J. Math. 123 (2001), no. 6, 1135-1169. MR.1867314

[58] E. Dobrowolski, On a question of Lehmer and the number of irreducible factors of a polynomial, Acta Arith. 34 (1979), no. 4, 391-401, DOI 10.4064/aa-34-4-391-401. MR543210

[59] John R. Doyle, Dynamical modular curves for quadratic polynomial maps (to appear Trans. Amer. Math. Soc.), https://doi.org/10.1090/tran/7474 (2018).

[60] John R. Doyle, Holly Krieger, Andrew Obus, Rachel Pries, Simon Rubinstein-Salzedo, and Lloyd W. West, Reduction of dynatomic curves (to appear Ergodic Theory Dynam. Systems), https://doi.org/10.1017/etds.2017.140 (2018).

[61] John R. Doyle and Bjorn Poonen, Gonality of dynatomic curves and strong uniform boundedness of preperiodic points, arXiv:1711.04233 (2017).

[62] John R. Doyle and Joseph H. Silverman, Moduli spaces for dynamcial systems with portraits, arXiv:1812.09936 2018 .

[63] John R. Doyle and Joseph H. Silverman, A uniform field-of-definition/field-of-moduli bound for dynamical systems on $\mathbb{P}^{N}$, J. Number Theory 195 (2019), 1-22, DOI 10.1016/j.jnt.2018.08.011. MR3867431

[64] Xander Faber and Andrew Granville, Prime factors of dynamical sequences, J. Reine Angew. Math. 661 (2011), 189-214, DOI 10.1515/CRELLE.2011.081. MR2863906

[65] Najmuddin Fakhruddin, Boundedness results for periodic points on algebraic varieties, Proc. Indian Acad. Sci. Math. Sci. 111 (2001), no. 2, 173-178, DOI 10.1007/BF02829589. MR 1836365

[66] Najmuddin Fakhruddin, Questions on self maps of algebraic varieties, J. Ramanujan Math. Soc. 18 (2003), no. 2, 109-122. MR.1995861

[67] G. Faltings, Endlichkeitssätze für abelsche Varietäten über Zahlkörpern (German), Invent. Math. 73 (1983), no. 3, 349-366, DOI 10.1007/BF01388432. MR718935

[68] Gerd Faltings, Diophantine approximation on abelian varieties, Ann. of Math. (2) 133 (1991), no. 3, 549-576, DOI 10.2307/2944319. MR 1109353

[69] Gerd Faltings, The general case of S. Lang's conjecture, Barsotti Symposium in Algebraic Geometry (Abano Terme, 1991), Perspect. Math., vol. 15, Academic Press, San Diego, CA, 1994, pp. 175-182. MR 1307396

[70] Charles Favre and Thomas Gauthier, Classification of special curves in the space of cubic polynomials, Int. Math. Res. Not. IMRN 2 (2018), 362-411, DOI 10.1093/imrn/rnw245. MR.3801434

[71] Charles Favre and Mattias Jonsson, Eigenvaluations (English, with English and French summaries), Ann. Sci. École Norm. Sup. (4) 40 (2007), no. 2, 309-349, DOI 10.1016/j.ansens.2007.01.002. MR2339287

[72] Charles Favre and Mattias Jonsson, Dynamical compactifications of $\mathbf{C}^{2}$, Ann. of Math. (2) 173 (2011), no. 1, 211-248, DOI 10.4007/annals.2011.173.1.6. MR.2753603

[73] Charles Favre and Juan Rivera-Letelier, Théorème d'équidistribution de Brolin en dynamique p-adique (French, with English and French summaries), C. R. Math. Acad. Sci. Paris 339 (2004), no. 4, 271-276, DOI 10.1016/j.crma.2004.06.023. MR2092012

[74] Charles Favre and Juan Rivera-Letelier, Équidistribution quantitative des points de petite hauteur sur la droite projective, Math. Ann., 335 (2006), 311-361; Corrigendum, Math. Ann. 339 (2007), 799-801.

[75] Charles Favre and Juan Rivera-Letelier, Théorie ergodique des fractions rationnelles sur un corps ultramétrique (French, with English and French summaries), Proc. Lond. Math. Soc. (3) 100 (2010), no. 1, 116-154, DOI 10.1112/plms/pdp022. MR2578470

[76] Charles Favre and Elizabeth Wulcan, Degree growth of monomial maps and McMullen's polytope algebra, Indiana Univ. Math. J. 61 (2012), no. 2, 493-524, DOI 10.1512/iumj.2012.61.4555. MR 3043585 
[77] Andrea Ferraguti, The set of stable primes for polynomial sequences with large Galois group, Proc. Amer. Math. Soc. 146 (2018), no. 7, 2773-2784, DOI 10.1090/proc/13958. MR.3787342

[78] Andrea Ferraguti, Giacomo Micheli, and Reto Schnyder, On sets of irreducible polynomials closed by composition, Arithmetic of finite fields, Lecture Notes in Comput. Sci., vol. 10064, Springer, Cham, 2016, pp. 77-83. MR3649090

[79] Andrea Ferraguti, Giacomo Micheli, and Reto Schnyder, Irreducible compositions of degree two polynomials over finite fields have regular structure, Q. J. Math. 69 (2018), no. 3, 1089-1099, DOI 10.1093/qmath/hay015. MR.3859225

[80] Philippe Flajolet and Andrew M. Odlyzko, Random mapping statistics, Advances in cryptology-EUROCRYPT '89 (Houthalen, 1989), Lecture Notes in Comput. Sci., vol. 434, Springer, Berlin, 1990, pp. 329-354, DOI 10.1007/3-540-46885-4_34. MR1083961

[81] E. V. Flynn, Bjorn Poonen, and Edward F. Schaefer, Cycles of quadratic polynomials and rational points on a genus-2 curve, Duke Math. J. 90 (1997), no. 3, 435-463, DOI 10.1215/S0012-7094-97-09011-6. MR.1480542

[82] Ryan Flynn and Derek Garton, Graph components and dynamics over finite fields, Int. J. Number Theory 10 (2014), no. 3, 779-792, DOI 10.1142/S1793042113501224. MR3190008

[83] John Erik Fornæss and Nessim Sibony, Complex dynamics in higher dimension. I, Astérisque 222 (1994), 5, 201-231. Complex analytic methods in dynamical systems (Rio de Janeiro, 1992). MR 1285389

[84] Thomas Gauthier, Yusuke Okuyama, and Gabriel Vigny, Approximation of non-archimedean Lyapunov exponents and applications over global fields, arXiv:1803.06859 (2018).

[85] D. Ghioca and T. J. Tucker, A dynamical version of the Mordell-Lang conjecture for the additive group, Compos. Math. 144 (2008), no. 2, 304-316, DOI 10.1112/S0010437X07003260. MR.2406114

[86] D. Ghioca and T. J. Tucker, Periodic points, linearizing maps, and the dynamical Mordell-Lang problem, J. Number Theory 129 (2009), no. 6, 1392-1403, DOI 10.1016/j.jnt.2008.09.014. MR2521481

[87] Dragos Ghioca, The dynamical Mordell-Lang conjecture, CMS Notes 46 (2014), no. 3, 14-15. MR 3235993

[88] Dragos Ghioca, The dynamical Mordell-Lang conjecture in positive characteristic, Trans. Amer. Math. Soc. 371 (2019), no. 2, 1151-1167, DOI 10.1090/tran/7261. MR3885174

[89] Dragos Ghioca, Liang-Chung Hsia, and Thomas J. Tucker, Preperiodic points for families of polynomials, Algebra Number Theory 7 (2013), no. 3, 701-732, DOI 10.2140/ant.2013.7.701. MR.3095224

[90] Dragos Ghioca, Holly Krieger, and Khoa Nguyen, A case of the dynamical André-Oort conjecture, Int. Math. Res. Not. IMRN 3 (2016), 738-758, DOI 10.1093/imrn/rnv143. MR.3493432

[91] Dragos Ghioca, Khoa Nguyen, and Hexi Ye, The dynamical Manin-Mumford conjecture and the dynamical Bogomolov conjecture for split rational maps, arXiv:1511.06081 (2015),

[92] Dragos Ghioca, Khoa D. Nguyen, and Hexi Ye, The dynamical Manin-Mumford conjecture and the dynamical Bogomolov conjecture for endomorphisms of $\left(\mathbb{P}^{1}\right)^{n}$, Compos. Math. 154 (2018), no. 7, 1441-1472, DOI 10.1112/s0010437x18007157. MR3826461

[93] Dragos Ghioca, Thomas Tucker, and Michael E. Zieve, The Mordell-Lang question for endomorphisms of semiabelian varieties (English, with English and French summaries), J. Théor. Nombres Bordeaux 23 (2011), no. 3, 645-666, DOI 10.5802/jtnb.781. MR2861079

[94] Dragos Ghioca, Thomas J. Tucker, and Shouwu Zhang, Towards a dynamical Manin-Mumford conjecture, Int. Math. Res. Not. IMRN 22 (2011), 5109-5122, DOI 10.1093/imrn/rnq283. MR2854724

[95] Dragos Ghioca, Thomas J. Tucker, and Michael E. Zieve, Intersections of polynomials orbits, and a dynamical Mordell-Lang conjecture, Invent. Math. 171 (2008), no. 2, 463-483, DOI 10.1007/s00222-007-0087-5. MR2367026

[96] Dragos Ghioca and Hexi Ye, A dynamical variant of the André-Oort conjecture, Int. Math. Res. Not. IMRN 8 (2018), 2447-2480, DOI 10.1093/imrn/rnw314. MR3801489

[97] Vefa Goksel, On the orbit of a post-critically finite polynomial of the form $x^{d}+c$, arXiv: 1806.01208 (2018).

[98] Vefa Goksel, Shixiang Xia, and Nigel Boston, A refined conjecture for factorizations of iterates of quadratic polynomials over finite fields, Exp. Math. 24 (2015), no. 3, 304-311, DOI 10.1080/10586458.2014.992079. MR3359218 
[99] Domingo Gomez and Alejandro P. Nicolás, An estimate on the number of stable quadratic polynomials, Finite Fields Appl. 16 (2010), no. 6, 401-405, DOI 10.1016/j.ffa.2010.06.005. MR2727344

[100] Domingo Gómez-Pérez, Alina Ostafe, and Igor E. Shparlinski, On irreducible divisors of iterated polynomials, Rev. Mat. Iberoam. 30 (2014), no. 4, 1123-1134, DOI 10.4171/RMI/809. MR.3293428

[101] Richard Gottesman and Kwokfung Tang, Quadratic recurrences with a positive density of prime divisors, Int. J. Number Theory 6 (2010), no. 5, 1027-1045, DOI 10.1142/S1793042110003368. MR2679455

[102] C. Gratton, K. Nguyen, and T. J. Tucker, $A B C$ implies primitive prime divisors in arithmetic dynamics, Bull. Lond. Math. Soc. 45 (2013), no. 6, 1194-1208, DOI 10.1112/blms/bdt049. MR 3138487

[103] Vincent Guedj, Ergodic properties of rational mappings with large topological degree, Ann. of Math. (2) 161 (2005), no. 3, 1589-1607, DOI 10.4007/annals.2005.161.1589. MR2179389

[104] Spencer Hamblen, Rafe Jones, and Kalyani Madhu, The density of primes in orbits of $z^{d}+c$, Int. Math. Res. Not. IMRN 7 (2015), 1924-1958, DOI 10.1093/imrn/rnt349. MR3335237

[105] Bernard Harris, Probability distributions related to random mappings, Ann. Math. Statist. 31 (1960), 1045-1062, DOI 10.1214/aoms/1177705677. MR0119227

[106] Boris Hasselblatt and James Propp, Degree-growth of monomial maps, Ergodic Theory Dynam. Systems 27 (2007), no. 5, 1375-1397, DOI 10.1017/S0143385707000168. MR2358970

[107] D. R. Heath-Brown, Iteration of quadratic polynomials over finite fields, Mathematika 63 (2017), no. 3, 1041-1059, DOI 10.1112/S0025579317000328. MR3731313

[108] D.R. Heath-Brown and Giacomo Micheli, Irreducible polynomials over finite fields produced by composition of quadratics, arXiv:1701.05031 (2017).

[109] M. Herman and J.-C. Yoccoz, Generalizations of some theorems of small divisors to nonArchimedean fields, Geometric dynamics (Rio de Janeiro, 1981), Lecture Notes in Math., vol. 1007, Springer, Berlin, 1983, pp. 408-447, DOI 10.1007/BFb0061427. MR730280

[110] Rubén A. Hidalgo, A simple remark on the field of moduli of rational maps, Q. J. Math. 65 (2014), no. 2, 627-635, DOI 10.1093/qmath/hat012. MR3230378

[111] Wade Hindes and Rafe Jones, Riccati equations and polynomial dynamics over function fields, arXiv:1710.04332 (2017).

[112] Marc Hindry and Joseph H. Silverman, Diophantine geometry: An introduction, Graduate Texts in Mathematics, vol. 201, Springer-Verlag, New York, 2000. MR.1745599

[113] Liang-Chung Hsia, Closure of periodic points over a non-Archimedean field, J. London Math. Soc. (2) 62 (2000), no. 3, 685-700, DOI 10.1112/S0024610700001447. MR.1794277

[114] Liang-Chung Hsia and Joseph Silverman, On a dynamical Brauer-Manin obstruction (English, with English and French summaries), J. Théor. Nombres Bordeaux 21 (2009), no. 1, 235-250. MR2537714

[115] Benjamin Hutz, Good reduction of periodic points on projective varieties, Illinois J. Math. 53 (2009), no. 4, 1109-1126. MR2741181

[116] Benjamin Hutz, Dynatomic cycles for morphisms of projective varieties, New York J. Math. 16 (2010), 125-159. MR 2657371

[117] Benjamin Hutz, Effectivity of dynatomic cycles for morphisms of projective varieties using deformation theory, Proc. Amer. Math. Soc. 140 (2012), no. 10, 3507-3514, DOI 10.1090/S0002-9939-2012-11192-X. MR2929019

[118] Patrick Ingram, Lower bounds on the canonical height associated to the morphism $\phi(z)=z^{d}+c$, Monatsh. Math. 157 (2009), no. 1, 69-89, DOI 10.1007/s00605-008-00186. MR2504779

[119] Patrick Ingram, Variation of the canonical height for a family of polynomials, J. Reine Angew. Math. 685 (2013), 73-97. MR3181564

[120] Patrick Ingram, Variation of the canonical height for polynomials in several variables, Int. Math. Res. Not. IMRN 24 (2015), 13545-13562, DOI 10.1093/imrn/rnv121. MR3436155

[121] Patrick Ingram, The critical height is a moduli height, Duke Math. J. 167 (2018), no. 7, 1311-1346, DOI 10.1215/00127094-2017-0053. MR3799700

[122] Patrick Ingram and Joseph H. Silverman, Primitive divisors in arithmetic dynamics, Math. Proc. Cambridge Philos. Soc. 146 (2009), no. 2, 289-302, DOI 10.1017/S0305004108001795. MR 2475968 
[123] Rafe Jones, The density of prime divisors in the arithmetic dynamics of quadratic polynomials, J. Lond. Math. Soc. (2) 78 (2008), no. 2, 523-544, DOI 10.1112/jlms/jdn034. MR2439638

[124] Rafe Jones, An iterative construction of irreducible polynomials reducible modulo every prime, J. Algebra 369 (2012), 114-128, DOI 10.1016/j.jalgebra.2012.05.020. MR2959789

[125] Rafe Jones, Galois representations from pre-image trees: an arboreal survey (English, with English and French summaries), Actes de la Conférence "Théorie des Nombres et Applications", Publ. Math. Besançon Algèbre Théorie Nr., vol. 2013, Presses Univ. Franche-Comté, Besançon, 2013, pp. 107-136. MR3220023

[126] Rafe Jones and Nigel Boston, Settled polynomials over finite fields, Proc. Amer. Math. Soc. 140 (2012), no. 6, 1849-1863, DOI 10.1090/S0002-9939-2011-11054-2. MR2888174

[127] Rafe Jones and Alon Levy, Eventually stable rational functions, Int. J. Number Theory 13 (2017), no. 9, 2299-2318, DOI 10.1142/S1793042117501263. MR3704363

[128] Rafe Jones and Michelle Manes, Galois theory of quadratic rational functions, Comment. Math. Helv. 89 (2014), no. 1, 173-213, DOI 10.4171/CMH/316. MR3177912

[129] Mattias Jonsson and Paul Reschke, On the complex dynamics of birational surface maps defined over number fields, J. Reine Angew. Math. 744 (2018), 275-297, DOI 10.1515/crelle2015-0113. MR3871447

[130] Mattias Jonsson and Elizabeth Wulcan, Canonical heights for plane polynomial maps of small topological degree, Math. Res. Lett. 19 (2012), no. 6, 1207-1217, DOI 10.4310/MRL.2012.v19.n6.a3. MR3091603

[131] Jamie Juul, Pär Kurlberg, Kalyani Madhu, and Tom J. Tucker, Wreath products and proportions of periodic points, Int. Math. Res. Not. IMRN 13 (2016), 3944-3969, DOI 10.1093/imrn/rnv273. MR3544625

[132] Borys Kadets, Large arboreal Galois representations, arXiv:1802.09074 (2018).

[133] S. Kamienny, Torsion points on elliptic curves and q-coefficients of modular forms, Invent. Math. 109 (1992), no. 2, 221-229, DOI 10.1007/BF01232025. MR.1172689

[134] Shu Kawaguchi and Joseph H. Silverman, Canonical heights and the arithmetic complexity of morphisms on projective space, Pure Appl. Math. Q. 5 (2009), no. 4, Special Issue: In honor of John Tate., 1201-1217, DOI 10.4310/PAMQ.2009.v5.n4.a2. MR2560315

[135] Shu Kawaguchi and Joseph H. Silverman, Examples of dynamical degree equals arithmetic degree, Michigan Math. J. 63 (2014), no. 1, 41-63, DOI 10.1307/mmj/1395234358. MR.3189467

[136] Shu Kawaguchi and Joseph H. Silverman, Dynamical canonical heights for Jordan blocks, arithmetic degrees of orbits, and nef canonical heights on abelian varieties, Trans. Amer. Math. Soc. 368 (2016), no. 7, 5009-5035, DOI 10.1090/tran/6596. MR3456169

[137] Shu Kawaguchi and Joseph H. Silverman, On the dynamical and arithmetic degrees of rational self-maps of algebraic varieties, J. Reine Angew. Math. 713 (2016), 21-48, DOI 10.1515/crelle-2014-0020. MR3483624

[138] Sergei V. Konyagin, Florian Luca, Bernard Mans, Luke Mathieson, Min Sha, and Igor E. Shparlinski, Functional graphs of polynomials over finite fields, J. Combin. Theory Ser. B 116 (2016), 87-122, DOI 10.1016/j.jctb.2015.07.003. MR3425238

[139] Holly Krieger, Primitive prime divisors in the critical orbit of $z^{d}+c$, Int. Math. Res. Not. IMRN 23 (2013), 5498-5525, DOI 10.1093/imrn/rns213. MR3142262

[140] David Krumm, A local-global principle in the dynamics of quadratic polynomials, Int. J. Number Theory 12 (2016), no. 8, 2265-2297, DOI 10.1142/S1793042116501360. MR3562026

[141] Martin D. Kruskal, The expected number of components under a random mapping function, Amer. Math. Monthly 61 (1954), 392-397, DOI 10.2307/2307900. MR0062973

[142] Serge Lang, Elliptic curves: Diophantine analysis, Grundlehren der Mathematischen Wissenschaften [Fundamental Principles of Mathematical Sciences], vol. 231, Springer-Verlag, Berlin-New York, 1978. MR518817

[143] Serge Lang, Fundamentals of Diophantine geometry, Springer-Verlag, New York, 1983. MR715605

[144] E. Lau and D. Schleicher, Internal addresses in the Mandelbrot set and irreducibility of polynomials, Technical Report 1994/19, (December 1994).

[145] Michel Laurent, Minoration de la hauteur de Néron-Tate (French), Seminar on number theory, Paris 1981-82 (Paris, 1981/1982), Progr. Math., vol. 38, Birkhäuser Boston, Boston, MA, 1983, pp. 137-151. MR729165 
[146] Chong Gyu Lee, The numerical equivalence relation for height functions and ampleness and nefness criteria for divisors, Bull. Lond. Math. Soc. 44 (2012), no. 5, 944-960, DOI 10.1112/blms/bds023. MR2975153

[147] John Lesieutre and Daniel Litt, Dynamical Mordell-Lang and automorphisms of blow-ups, arXiv:1604.08216 (2016).

[148] John Lesieutre and Matthew Satriano, Canonical heights on hyper-Kähler varieties and the Kawaguchi-Silverman conjecture, arXiv: 1802.07388 (2018).

[149] John Lesieutre and Matthew Satriano, A rational map with infinitely many points of distinct arithmetic degrees, arXiv:1809.00047 (2018).

[150] Alon Levy, The space of morphisms on projective space, Acta Arith. 146 (2011), no. 1, 13-31, DOI 10.4064/aa146-1-2. MR2741188

[151] Rudolf Lidl and Gary L. Mullen, Unsolved Problems: When Does a Polynomial Over a Finite Field Permute the Elements of the Field?, Amer. Math. Monthly 95 (1988), no. 3, 243-246, DOI 10.2307/2323626. MR 1541277

[152] Rudolf Lidl and Gary L. Mullen, Unsolved Problems: When Does a Polynomial over a Finite Field Permute the Elements of the Field?, II, Amer. Math. Monthly 100 (1993), no. 1, 71-74, DOI $10.2307 / 2324822$. MR 1542258

[153] Rudolf Lidl and Harald Niederreiter, Finite fields, 2nd ed., Encyclopedia of Mathematics and its Applications, vol. 20, Cambridge University Press, Cambridge, 1997. With a foreword by P. M. Cohn. MR1429394

[154] Jan-Li Lin, Algebraic stability and degree growth of monomial maps, Math. Z. 271 (2012), no. 1-2, 293-311, DOI 10.1007/s00209-011-0864-0. MR2917145

[155] Jan-Li Lin, Pulling back cohomology classes and dynamical degrees of monomial maps, Bull. Soc. Math. France 140 (2012), no. 4, 533-549 (2013), DOI 10.24033/bsmf.2635. MR3059849

[156] D. A. Lind and T. Ward, Automorphisms of solenoids and p-adic entropy, Ergodic Theory Dynam. Systems 8 (1988), no. 3, 411-419, DOI 10.1017/S0143385700004545. MR961739

[157] Nicole R. Looper, Dynamical Galois groups of trinomials and Odoni's conjecture (to appear Bull. Lond. Math. Soc.), arXiv:1609.03398 (2016).

[158] Nicole R. Looper, The abc-conjecture implies uniform bounds on dynamical Zsigmondy sets, arXiv:1711.01507 (2017).

[159] Nicole R. Looper, A lower bound on the canonical height for polynomials (to appear Math. Annalen), arXiv:1709.08121 (2017).

[160] Jonathan Lubin, Non-Archimedean dynamical systems, Compositio Math. 94 (1994), no. 3, 321-346. MR 1310863

[161] Michelle Manes, $\mathbb{Q}$-rational cycles for degree-2 rational maps having an automorphism, Proc. Lond. Math. Soc. (3) 96 (2008), no. 3, 669-696, DOI 10.1112/plms/pdm044. MR2407816

[162] Michelle Manes, Moduli spaces for families of rational maps on $\mathbb{P}^{1}$, J. Number Theory 129 (2009), no. 7, 1623-1663, DOI 10.1016/j.jnt.2009.02.010. MR2524186

[163] Michelle Manes and Joseph H. Silverman, A classification of degree 2 semi-stable rational maps $\mathbb{P}^{2} \rightarrow \mathbb{P}^{2}$ with large finite dynamical automorphism group (to appear Annales de la Faculte des Sciences de Toulouse), arxiv:1607.05772 (2016).

[164] Bernard Mans, Min Sha, Igor E. Shparlinski, and Daniel Sutantyo, On functional graphs of quadratic polynomials, Exp. Math. 0 (2017), no. 0, 1-9.

[165] D. Masser and U. Zannier, Torsion anomalous points and families of elliptic curves, Amer. J. Math. 132 (2010), no. 6, 1677-1691. MR2766181

[166] D. W. Masser, Counting points of small height on elliptic curves (English, with French summary), Bull. Soc. Math. France 117 (1989), no. 2, 247-265. MR.1015810

[167] Yohsuke Matsuzawa, On upper bounds of arithmetic degrees, arXiv:1606.00598 (2016).

[168] Yohsuke Matsuzawa, Kaoru Sano, and Takahiro Shibata, Arithmetic degrees and dynamical degrees of endomorphisms on surfaces, Algebra Number Theory 12 (2018), no. 7, 1635-1657, DOI 10.2140/ant.2018.12.1635. MR3871505

[169] Niki Myrto Mavraki and Hexi Ye, Quasi-adelic measures and equidistribution on $\mathbb{P}^{1}$, arXiv: 1502.04660 (2017).

[170] B. Mazur, Modular curves and the Eisenstein ideal, Inst. Hautes Études Sci. Publ. Math. 47 (1977), 33-186 (1978). MR488287

[171] Alice Medvedev and Thomas Scanlon, Polynomial dynamics, arXiv:0901.2352 (2009).

[172] Loï Merel, Bornes pour la torsion des courbes elliptiques sur les corps de nombres (French), Invent. Math. 124 (1996), no. 1-3, 437-449, DOI 10.1007/s002220050059. MR1369424 
[173] Nikita Miasnikov, Brian Stout, and Phillip Williams, Automorphism loci for the moduli space of rational maps, Acta Arith. 180 (2017), no. 3, 267-296, DOI 10.4064/aa8548-62017. MR 3709645

[174] John Milnor, Geometry and dynamics of quadratic rational maps, Experiment. Math. 2 (1993), no. 1, 37-83. With an appendix by the author and Lei Tan. MR.1246482

[175] John Milnor, Dynamics in one complex variable, 3rd ed., Annals of Mathematics Studies, vol. 160, Princeton University Press, Princeton, NJ, 2006. MR2193309

[176] John Milnor, On Lattès maps, Dynamics on the Riemann sphere, Eur. Math. Soc., Zürich, 2006, pp. 9-43, DOI 10.4171/011-1/1. MR2348953

[177] Patrick Morton, Arithmetic properties of periodic points of quadratic maps, Acta Arith. 62 (1992), no. 4, 343-372, DOI 10.4064/aa-62-4-343-372. MR1199627

[178] Patrick Morton, On certain algebraic curves related to polynomial maps, Compositio Math. 103 (1996), no. 3, 319-350. MR1414593

[179] Patrick Morton and Joseph H. Silverman, Rational periodic points of rational functions, Internat. Math. Res. Notices 2 (1994), 97-110, DOI 10.1155/S1073792894000127. MR1264933

[180] Patrick Morton and Joseph H. Silverman, Periodic points, multiplicities, and dynamical units, J. Reine Angew. Math. 461 (1995), 81-122, DOI 10.1515/crll.1995.461.81. MR.1324210

[181] D. G. Northcott, Periodic points on an algebraic variety, Ann. of Math. (2) 51 (1950), 167-177, DOI 10.2307/1969504. MR0034607

[182] R. W. K. Odoni, The Galois theory of iterates and composites of polynomials, Proc. London Math. Soc. (3) 51 (1985), no. 3, 385-414, DOI 10.1112/plms/s3-51.3.385. MR805714

[183] R. W. K. Odoni, On the prime divisors of the sequence $w_{n+1}=1+w_{1} \cdots w_{n}$, J. London Math. Soc. (2) 32 (1985), no. 1, 1-11, DOI 10.1112/jlms/s2-32.1.1. MR813379

[184] R. W. K. Odoni, Realising wreath products of cyclic groups as Galois groups, Mathematika 35 (1988), no. 1, 101-113, DOI 10.1112/S002557930000632X. MR962740

[185] Reinhard Oselies and Heiner Zieschang, Ergodische Eigenschaften der Automorphismen p-adischer Zahlen (German), Arch. Math. (Basel) 26 (1975), 144-153, DOI 10.1007/BF01229718. MR0369301

[186] Alina Ostafe and Min Sha, On the quantitative dynamical Mordell-Lang conjecture, J. Number Theory 156 (2015), 161-182, DOI 10.1016/j.jnt.2015.04.011. MR3360335

[187] Clayton Petsche, Critically separable rational maps in families, Compos. Math. 148 (2012), no. 6, 1880-1896, DOI 10.1112/S0010437X12000346. MR2999309

[188] Clayton Petsche and Brian Stout, On quadratic rational maps with prescribed good reduction, Proc. Amer. Math. Soc. 143 (2015), no. 3, 1145-1158, DOI 10.1090/S0002-9939-2014-12291X. MR.3293730

[189] Clayton Petsche, Lucien Szpiro, and Michael Tepper, Isotriviality is equivalent to potential good reduction for endomorphisms of $\mathbb{P}^{N}$ over function fields, J. Algebra 322 (2009), no. 9, 3345-3365, DOI 10.1016/j.jalgebra.2008.11.027. MR2567424

[190] T. Pezda, Cycles of polynomial mappings in several variables, Manuscripta Math. 83 (1994), no. 3-4, 279-289, DOI 10.1007/BF02567614. MR.1277530

[191] Bjorn Poonen, The classification of rational preperiodic points of quadratic polynomials over Q: a refined conjecture, Math. Z. 228 (1998), no. 1, 11-29, DOI 10.1007/PL00004405. MR.1617987

[192] Bjorn Poonen, Uniform boundedness of rational points and preperiodic points, arXiv:1206. 7104 (2012).

[193] M. Raynaud, Courbes sur une variété abélienne et points de torsion (French), Invent. Math. 71 (1983), no. 1, 207-233, DOI 10.1007/BF01393342. MR688265

[194] M. Raynaud, Sous-variétés d'une variété abélienne et points de torsion (French), Arithmetic and geometry, Vol. I, Progr. Math., vol. 35, Birkhäuser Boston, Boston, MA, 1983, pp. 327352. MR717600

[195] Juan Rivera-Letelier. Dynamique des fonctions rationnelles sur des corps locaux, PhD thesis, Universite de Paris XI, 2000.

[196] Juan Rivera-Letelier, Dynamique des fonctions rationnelles sur des corps locaux (French, with English and French summaries), Astérisque 287 (2003), xv, 147-230. Geometric methods in dynamics. II. MR 2040006 
[197] Juan Rivera-Letelier, Espace hyperbolique p-adique et dynamique des fonctions rationnelles (French, with English summary), Compositio Math. 138 (2003), no. 2, 199-231, DOI 10.1023/A:1026136530383. MR2018827

[198] Robert Rumely, The minimal resultant locus, Acta Arith. 169 (2015), no. 3, 251-290, DOI 10.4064/aa169-3-3. MR.3361223

[199] Alexander Russakovskii and Bernard Shiffman, Value distribution for sequences of rational mappings and complex dynamics, Indiana Univ. Math. J. 46 (1997), no. 3, 897-932, DOI 10.1512/iumj.1997.46.1441. MR.1488341

[200] Kaoru Sano, Growth rate of ample heights and the dynamical Mordell-Lang conjecture, Int. J. Number Theory 14 (2018), no. 10, 2673-2685, DOI 10.1142/S1793042118501610. MR.3869605

[201] Thomas Scanlon and Yu Yasufuku, Exponential-polynomial equations and dynamical return sets, Int. Math. Res. Not. IMRN 16 (2014), 4357-4367, DOI 10.1093/imrn/rnt081. MR 3250036

[202] Victor Scharaschkin, Local-global problems and the Brauer-Manin obstruction, ProQuest LLC, Ann Arbor, MI, 1999. Thesis (Ph.D.)-University of Michigan. MR2700328

[203] Dierk Schleicher, Internal addresses of the Mandelbrot set and Galois groups of polynomials, Arnold Math. J. 3 (2017), no. 1, 1-35, DOI 10.1007/s40598-016-0042-x. MR3646529

[204] Jean-Pierre Serre, Propriétés galoisiennes des points d'ordre fini des courbes elliptiques (French), Invent. Math. 15 (1972), no. 4, 259-331, DOI 10.1007/BF01405086. MR.0387283

[205] Jean-Pierre Serre, Abelian l-adic representations and elliptic curves, Research Notes in Mathematics, vol. 7, A K Peters, Ltd., Wellesley, MA, 1998. With the collaboration of Willem Kuyk and John Labute; Revised reprint of the 1968 original. MR 1484415

[206] Xuancheng Shao, Polynomial values modulo primes on average and sharpness of the larger sieve, Algebra Number Theory 9 (2015), no. 10, 2325-2346, DOI 10.2140/ant.2015.9.2325. MR 3437764

[207] Mitsuhiro Shishikura, On the quasiconformal surgery of rational functions, Ann. Sci. École Norm. Sup. (4) 20 (1987), no. 1, 1-29. MR892140

[208] Joseph H. Silverman, Good reduction and Shafarevich-type theorems for dynamical systems with portrait level structures, Pacific J. Math. 295 (2018), no. 1, 145-190, DOI 10.2140/pjm.2018.295.145. MR 3778330

[209] Joseph H. Silverman, Rational points on K3 surfaces: a new canonical height, Invent. Math. 105 (1991), no. 2, 347-373, DOI 10.1007/BF01232270. MR1115546

[210] Joseph H. Silverman, Integer points, Diophantine approximation, and iteration of rational maps, Duke Math. J. 71 (1993), no. 3, 793-829, DOI 10.1215/S0012-7094-93-07129-3. MR.1240603

[211] Joseph H. Silverman, The field of definition for dynamical systems on $\mathbf{P}^{1}$, Compositio Math. 98 (1995), no. 3, 269-304. MR1351830

[212] Joseph H. Silverman, Rational functions with a polynomial iterate, J. Algebra 180 (1996), no. 1, 102-110, DOI 10.1006/jabr.1996.0054. MR.1375568

[213] Joseph H. Silverman, The space of rational maps on $\mathbf{P}^{1}$, Duke Math. J. 94 (1998), no. 1, 41-77, DOI 10.1215/S0012-7094-98-09404-2. MR1635900

[214] Joseph H. Silverman, The arithmetic of dynamical systems, Graduate Texts in Mathematics, vol. 241, Springer, New York, 2007. MR2316407

[215] Joseph H. Silverman, Variation of periods modulo $p$ in arithmetic dynamics, New York J. Math. 14 (2008), 601-616. MR2448661

[216] Joseph H. Silverman, The arithmetic of elliptic curves, 2nd ed., Graduate Texts in Mathematics, vol. 106, Springer, Dordrecht, 2009. MR2514094

[217] Joseph H. Silverman, Height estimates for equidimensional dominant rational maps, J. Ramanujan Math. Soc. 26 (2011), no. 2, 145-163. MR2816785

[218] Joseph H. Silverman, Moduli spaces and arithmetic dynamics, CRM Monograph Series, vol. 30, American Mathematical Society, Providence, RI, 2012. MR2884382

[219] Joseph H. Silverman, Primitive divisors, dynamical Zsigmondy sets, and Vojta's conjecture, J. Number Theory 133 (2013), no. 9, 2948-2963, DOI 10.1016/j.jnt.2013.03.005. MR.3057058

[220] Joseph H. Silverman, Dynamical degree, arithmetic entropy, and canonical heights for dominant rational self-maps of projective space, Ergodic Theory Dynam. Systems 34 (2014), no. 2, 647-678, DOI 10.1017/etds.2012.144. MR3233709 
[221] Joseph H. Silverman, Arithmetic and dynamical degrees on abelian varieties (English, with English and French summaries), J. Théor. Nombres Bordeaux 29 (2017), no. 1, 151-167. MR.3614521

[222] Joseph H. Silverman and Gregory S. Call, Degeneration of dynamical degrees in families of maps, Acta Arith. 184 (2018), no. 2, 101-116, DOI 10.4064/aa8620-5-2017. MR3841148

[223] Joseph H. Silverman and Bianca Viray, On a uniform bound for the number of exceptional linear subvarieties in the dynamical Mordell-Lang conjecture, Math. Res. Lett. 20 (2013), no. 3, 547-566, DOI 10.4310/MRL.2013.v20.n3.a12. MR3162847

[224] Joseph H. Silverman and José Felipe Voloch, A local-global criterion for dynamics on $\mathbb{P}^{1}$, Acta Arith. 137 (2009), no. 3, 285-294, DOI 10.4064/aa137-3-8. MR2496466

[225] Vijay A. Sookdeo, Integer points in backward orbits, J. Number Theory 131 (2011), no. 7, 1229-1239, DOI 10.1016/j.jnt.2011.01.005. MR2782838

[226] Joel Specter, Polynomials with surjective arboreal galois representations exist in every degree, arXiv: 1803.00434 (2018).

[227] Michael Stoll, Galois groups over Q of some iterated polynomials, Arch. Math. (Basel) 59 (1992), no. 3, 239-244, DOI 10.1007/BF01197321. MR1174401

[228] Michael Stoll, Rational 6-cycles under iteration of quadratic polynomials, LMS J. Comput. Math. 11 (2008), 367-380, DOI 10.1112/S1461157000000644. MR2465796

[229] Brian Justin Stout, A dynamical Shafarevich theorem for twists of rational morphisms, Acta Arith. 166 (2014), no. 1, 69-80, DOI 10.4064/aa166-1-6. MR3273498

[230] Dennis Sullivan, Quasiconformal homeomorphisms and dynamics. I. Solution of the FatouJulia problem on wandering domains, Ann. of Math. (2) 122 (1985), no. 3, 401-418, DOI 10.2307/1971308. MR819553

[231] L. Szpiro and L. West, A dynamical Shafarevich theorem for rational maps over number fields and function fields, arXiv:1705.05489 (2017).

[232] Lucien Szpiro, Michael Tepper, and Phillip Williams, Semi-stable reduction implies minimality of the resultant, J. Algebra 397 (2014), 489-498, DOI 10.1016/j.jalgebra.2013.09.008. MR.3119234

[233] Lucien Szpiro and Thomas J. Tucker, A Shafarevich-Faltings theorem for rational functions, Pure Appl. Math. Q. 4 (2008), no. 3, Special Issue: In honor of Fedor Bogomolov., 715-728, DOI 10.4310/PAMQ.2008.v4.n3.a4. MR2435841

[234] J. Tate, Algorithm for determining the type of a singular fiber in an elliptic pencil, Modular functions of one variable, IV (Proc. Internat. Summer School, Univ. Antwerp, Antwerp, 1972), Springer, Berlin, 1975, pp. 33-52. Lecture Notes in Math., Vol. 476. MR0393039

[235] J. Tate, Variation of the canonical height of a point depending on a parameter, Amer. J. Math. 105 (1983), no. 1, 287-294, DOI 10.2307/2374389. MR692114

[236] E. Thiran, D. Verstegen, and J. Weyers, p-adic dynamics, J. Statist. Phys. 54 (1989), no. 3-4, 893-913, DOI 10.1007/BF01019780. MR988564

[237] Eugenio Trucco, Wandering Fatou components and algebraic Julia sets (English, with English and French summaries), Bull. Soc. Math. France 142 (2014), no. 3, 411-464, DOI 10.24033/bsmf.2670. MR3295719

[238] Emmanuel Ullmo, Positivité et discrétion des points algébriques des courbes (French), Ann. of Math. (2) 147 (1998), no. 1, 167-179, DOI 10.2307/120987. MR1609514

[239] Ming-Xi Wang, A dynamical Mordell-Lang property on the disk, Trans. Amer. Math. Soc. 369 (2017), no. 3, 2183-2204, DOI 10.1090/tran/6775. MR 3581231

[240] Junyi Xie, Dynamical Mordell-Lang conjecture for birational polynomial morphisms on $\mathbb{A}^{2}$, Math. Ann. 360 (2014), no. 1-2, 457-480, DOI 10.1007/s00208-014-1039-1. MR3263169

[241] Junyi Xie, The dynamical Mordell-Lang conjecture for polynomial endomorphisms of the affine plane (English, with English and French summaries), Astérisque 394 (2017), vi+110. MR 3758955

[242] Junyi Xie, Periodic points of birational transformations on projective surfaces, Duke Math. J. 164 (2015), no. 5, 903-932, DOI 10.1215/00127094-2877402. MR3332894

[243] Junyi Xie, Algebraic dynamics of the lifts of Frobenius, Algebra Number Theory 12 (2018), no. 7, 1715-1748, DOI 10.2140/ant.2018.12.1715. MR3871508

[244] Junyi Xie, The existence of Zariski dense orbits for polynomial endomorphisms of the affine plane, Compos. Math. 153 (2017), no. 8, 1658-1672, DOI 10.1112/S0010437X17007187. MR.3705271 
[245] Shou-Wu Zhang, Equidistribution of small points on abelian varieties, Ann. of Math. (2) 147 (1998), no. 1, 159-165, DOI 10.2307/120986. MR 1609518

[246] Shou-Wu Zhang, Distributions in algebraic dynamics, Surveys in differential geometry. Vol. X, Surv. Differ. Geom., vol. 10, Int. Press, Somerville, MA, 2006, pp. 381-430, DOI 10.4310/SDG.2005.v10.n1.a9. MR2408228

[247] Shouwu Zhang, Small points and adelic metrics, J. Algebraic Geom. 4 (1995), no. 2, 281300. MR1311351

[248] Michael Ernest Zieve, Cycles of polynomial mappings, ProQuest LLC, Ann Arbor, MI, 1996. Thesis (Ph.D.)-University of California, Berkeley. MR2694837

[249] K. Zsigmondy, Zur Theorie der Potenzreste (German), Monatsh. Math. Phys. 3 (1892), no. 1, 265-284, DOI 10.1007/BF01692444. MR1546236

Department of Mathematics and Statistics, Amherst College, Amherst, MassachuSETTS 01002

Email address: rlbenedetto@amherst.edu

Department of Mathematics and Statistics, York University, N520 Ross, 4700 Keele Street, Toronto, Ontario M3J 1P3, Canada

Email address: pingram@yorku.ca

Carleton College, Department of Mathematics and Statistics, Northfield, MinNESOTA 55057

Email address: rfjones@carleton.edu

Department of Mathematics, University of Hawait, 2565 McCarthy Mall, Honolulu, HAWAII 96822

Email address: mmanes@math.hawaii.edu

Mathematics Department, Box 1917, Brown University, Providence, Rhode Island 02912

Email address: jhs@math.brown.edu

Mathematics Department, 915 Hylan Building, University of Rochester, Rochester, New York 14627

Email address: thomas.tucker@rochester.edu 